\title{
The development of a liquid cooling test system for the analysis of porous surfaces
}

By

Ben Sherson

A thesis submitted to Victoria University of Wellington in fulfilment of the requirements of the degree of Master of Engineering in Electrical Engineering

Victoria University of Wellington 



\section{Abstract}

Closed-loop liquid cooling systems are used in a wide variety of high temperature environments, as liquids tend a higher thermal conductivity than air. Microchannels and porous microstructures have proved to be useful in improving the cooling capabilities of cooling systems, due to their increased surface area in contact with the cooling fluid. This thesis describes the design, development, and evaluation of a closed-loop liquid cooling test system. This system was utilised in analysing the thermal properties of porous microstructures for use in improving cooling capabilities. Flow rate and pressure sensors were fitted onto a standard closed loop liquid cooling system design, and thermocouples were attached to the system to measure the temperature at various points, as well as measure heat flux. Using these measurements, the thermal and hydraulic resistances of the system could be calculated. Various substrates were fabricated using both freeze casting and other techniques, and the thermal and hydraulic resistances of these substrates were characterized using the test system. The test system performed very well, with results matching the trends as expected from theory. However, no improvement in heat transfer was observed from microstructured silver surfaces compared to a solid copper reference surface. This may be due to the formation of oxides and/or sulphides on these silver surfaces, resulting in a reduction in the convective heat transfer from these layers. 



\section{Acknowledgements}

First and foremost, I would like to thank my supervisor, Dr. Gideon Gouws. I am extremely grateful for his long-term support, and his guidance has been invaluable throughout this project. I do not doubt that without him I would not have made it this far, particularly given that he is the one who gave me the passion to pursue a masters in the first place.

Secondly, the Victoria University workshop team: Nick Grinter, Alan Rennie, and Alex Puglisi, strongly deserve my thanks. Their assistance in the fabrication of components was a practical necessity to the success of this project. Their advice on potential solutions to one of the problems encountered, that of the porous silver shrinking as it sintered, inspired the solution I eventually opted for.

Further thanks go to Tim Exley, Jason Edwards, Brandon Pothoven, and Joel Bancolita, for their assistance with technical issues. While their contributions to the project are not on the level of Dr. Gouws and the workshop team, they helped me keep moving smoothly instead of stopping at each minor roadblock, and I am grateful for that assistance.

Finally, I would like to thank my friends and family for their emotional support over the past year. Their words of support inspired me to dig into the project with fervour, and to knuckle down and keep going when things got tough. 



\section{Contents}

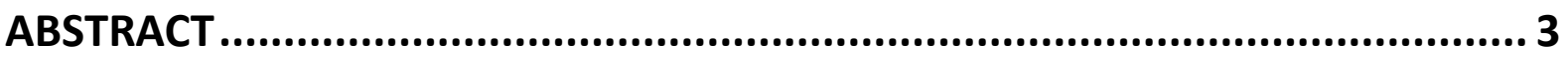

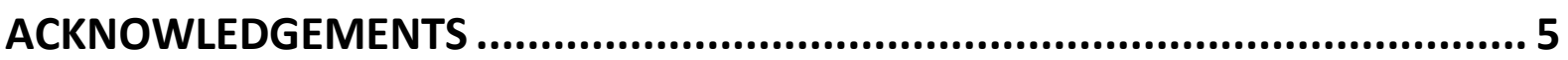

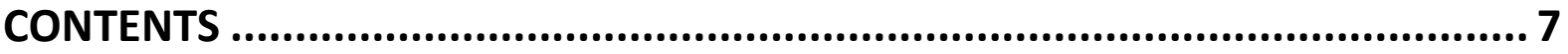

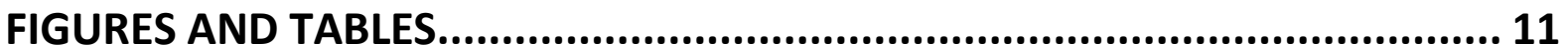

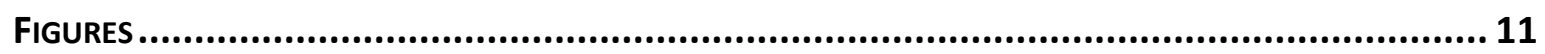

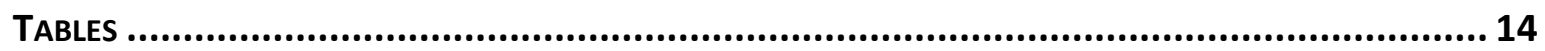

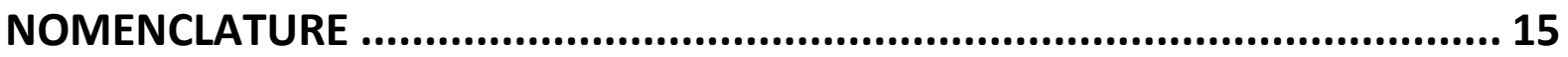

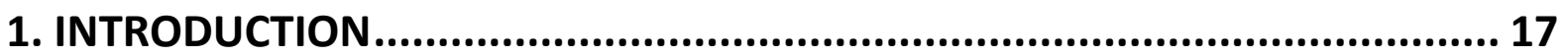

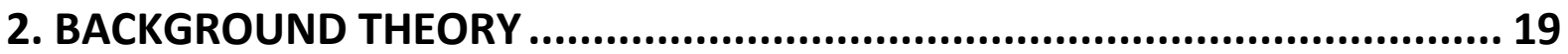

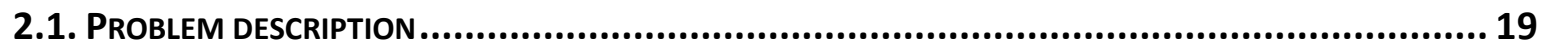

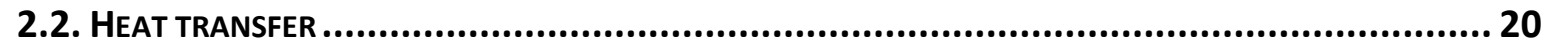

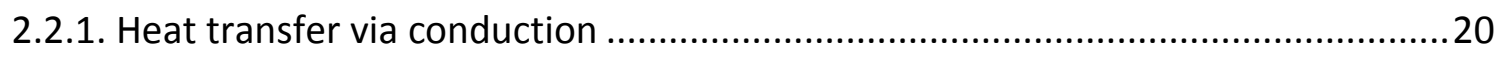

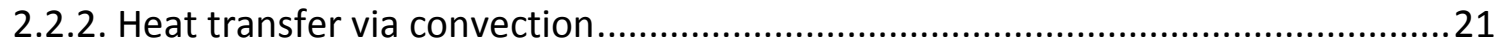

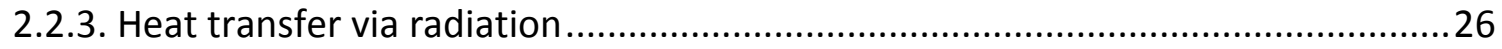

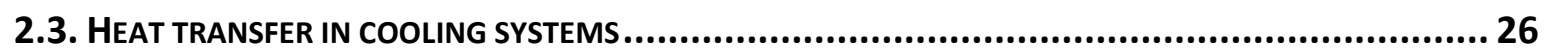

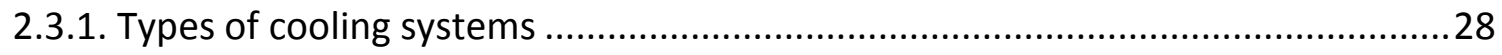

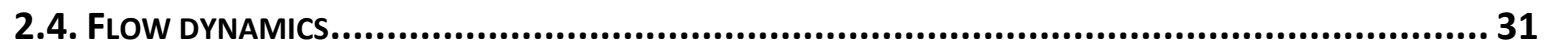

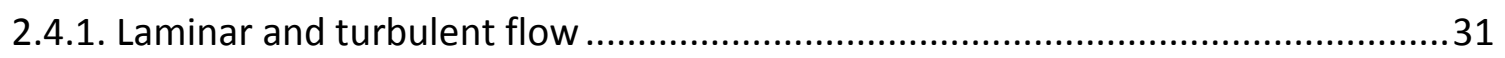

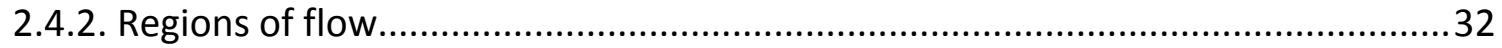

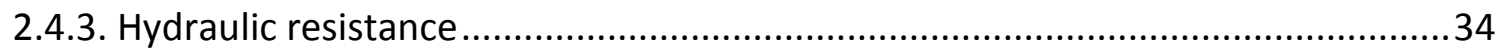

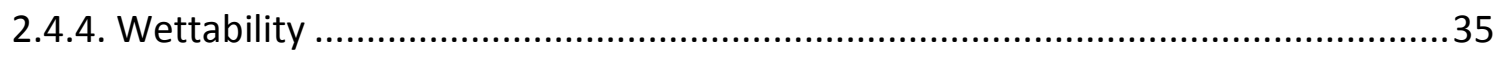

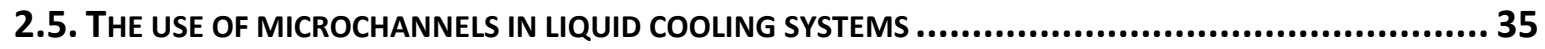

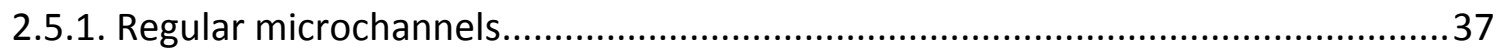

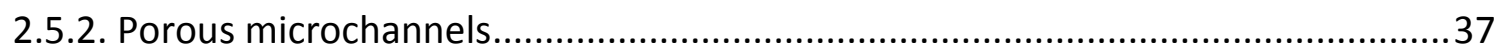

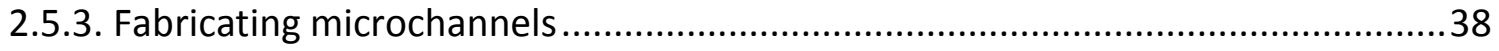


2.6. LITERATURE REVIEW OF POROUS MICROCHANNELS .................................................... 40

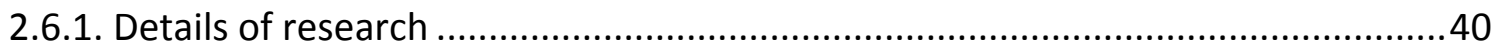

2.6.1.1. Chen et al: Two-phase film heating in bi-dispersed sintered copper .......................... 40

2.6.1.2. Jiang et al: Hot/cold water system with sintered copper particles............................ 41

2.6.1.3. Boomsma et al: Liquid cooled heater with compressed aluminium foam ................... 41

2.6.1.4. Jiang et al: Air and water cooling with sintered bronze particles ............................... 42

2.6.1.5. Zhang et al: Ball grid array die cooling with nickel-plated copper foam .................... 43

2.6.1.6. Hetsroni et al: Liquid cooling with sintered stainless steel ....................................... 43

2.6.1.7. Singh et al: Liquid cooling with sintered copper particles......................................... 43

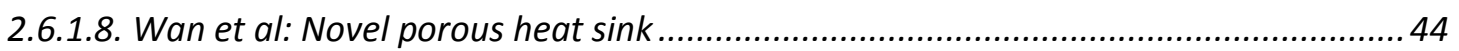

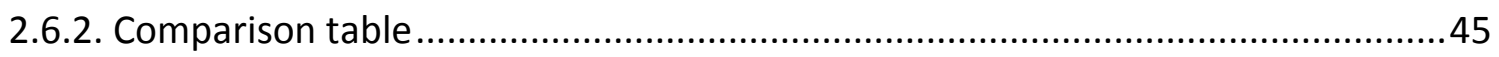

2.7. PREVIOUS WORK WITH POROUS SILVER AT VICTORIA UNIVERSITY ........................................ 46

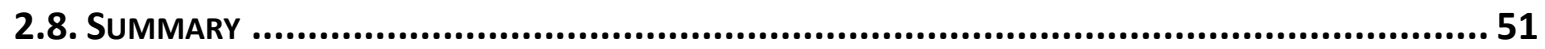

3. TEST SYSTEM FOR FORCED CONVECTIVE HEAT TRANSFER ........................53

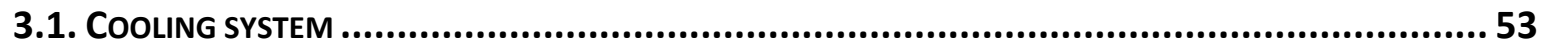

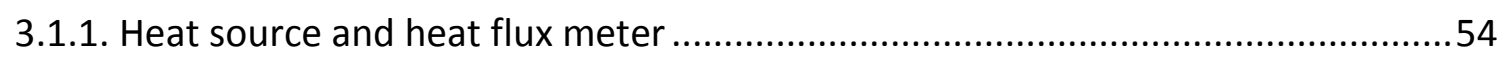

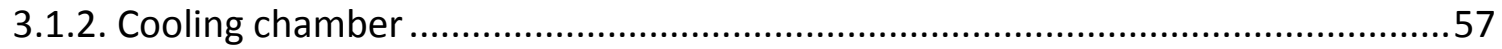

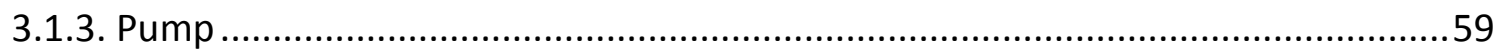

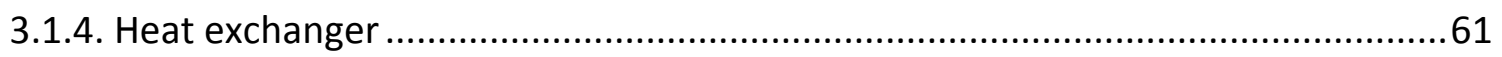

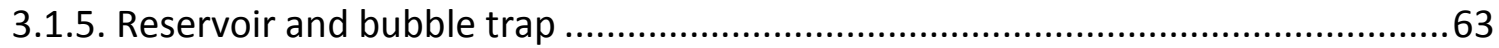

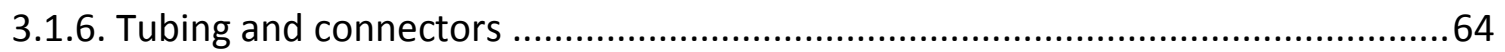

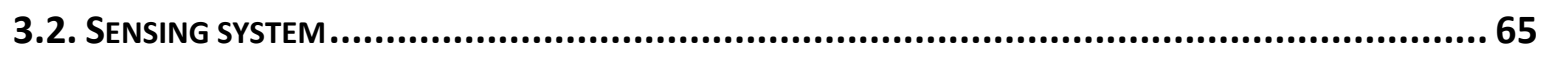

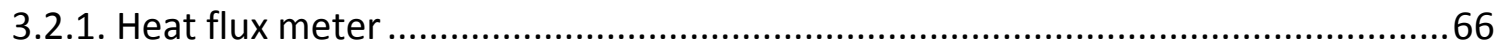

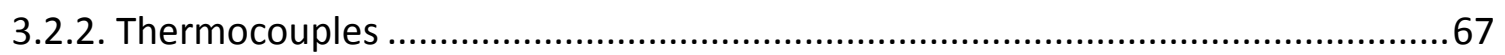

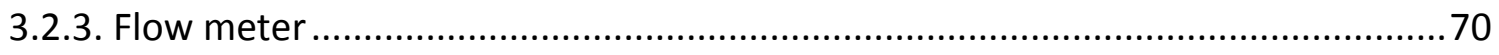

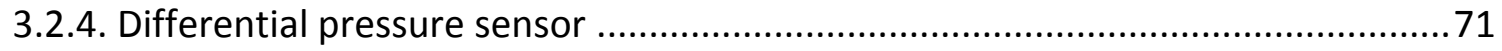

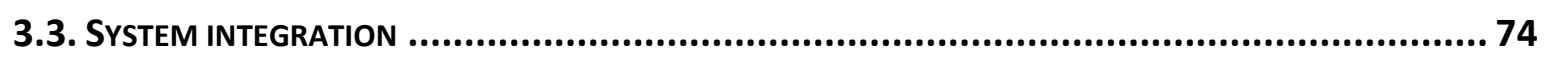

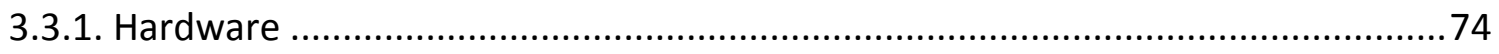

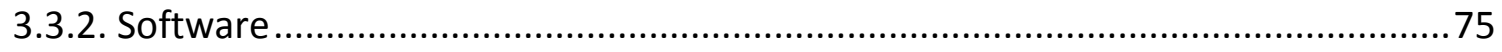

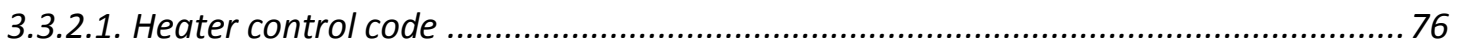




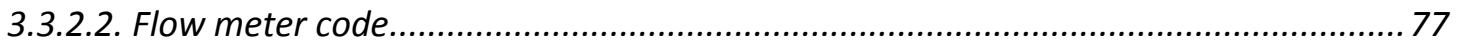

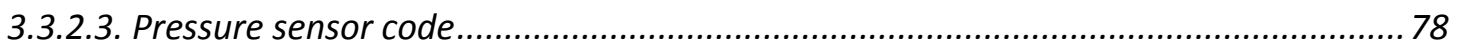

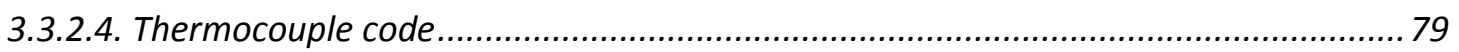

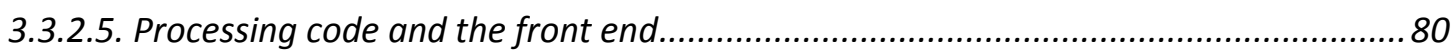

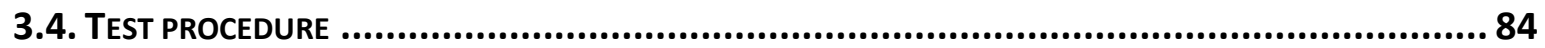

\section{FABRICATION AND PROPERTIES OF POROUS AND STRUCTURED SURFACES}

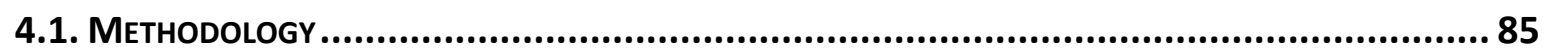

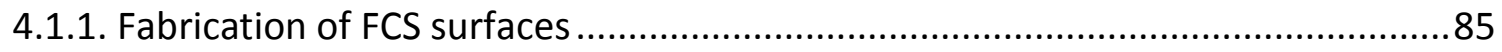

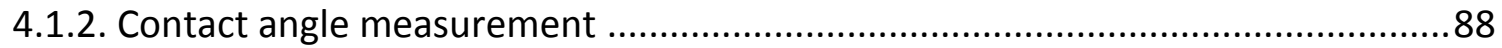

4.1.2.1. Contact angle measurement procedure …............................................................... 90

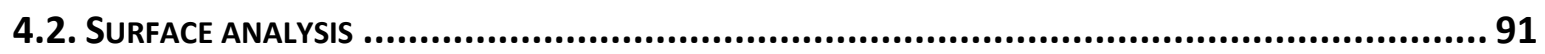

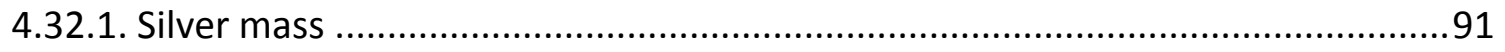

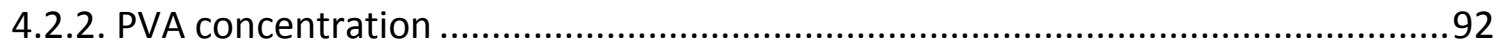

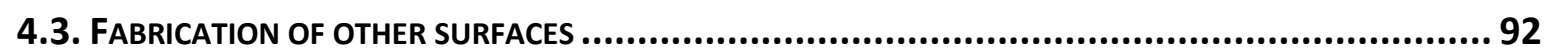

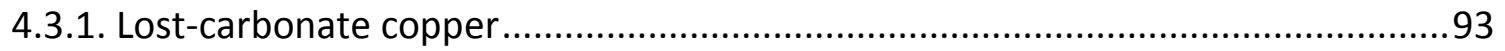

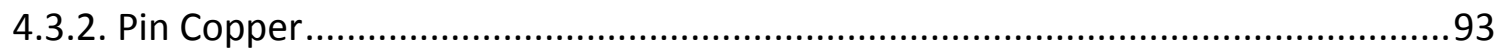

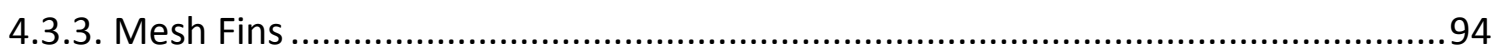

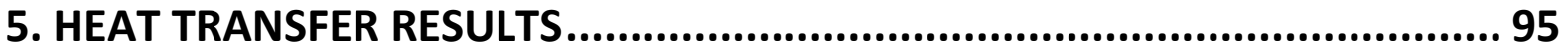

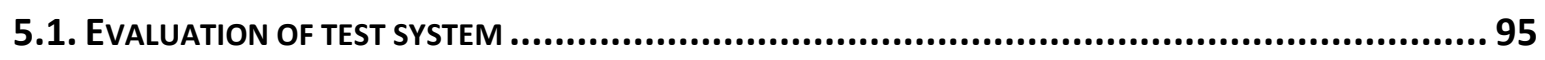

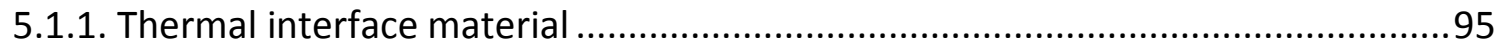

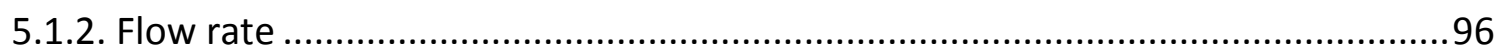

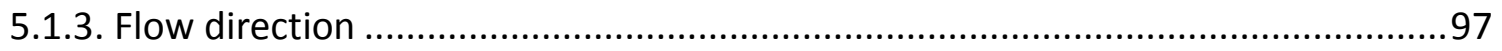

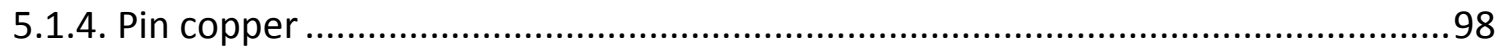

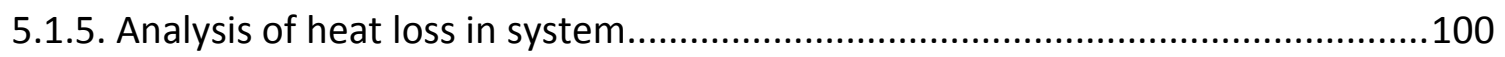

5.1.6. Test of a Corsair H110 commercial liquid cooling system .................................102

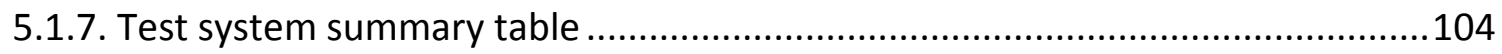

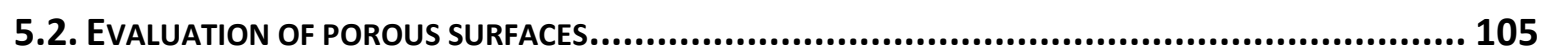

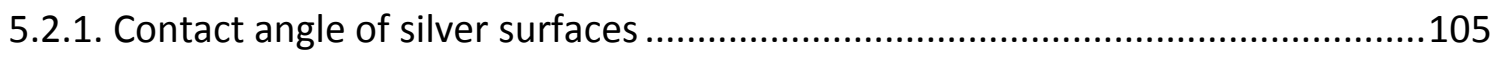

5.2.2. Performance of commercial porous copper material .......................................106 


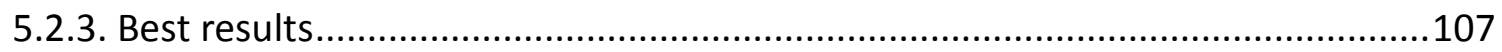

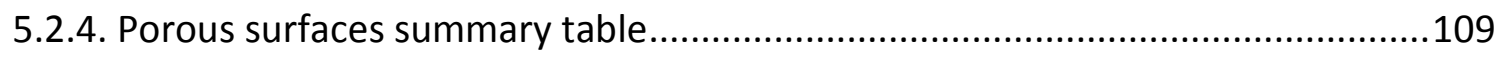

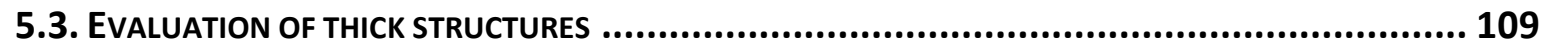

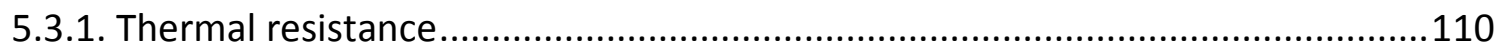

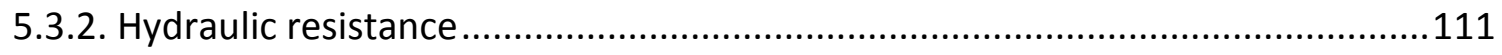

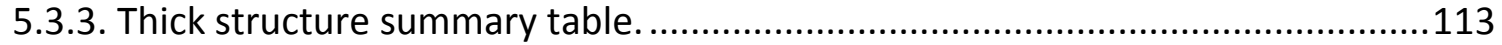

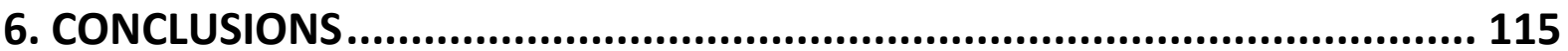

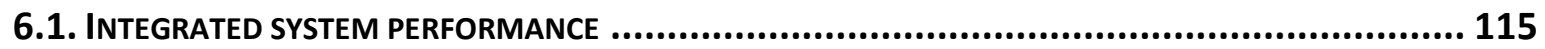

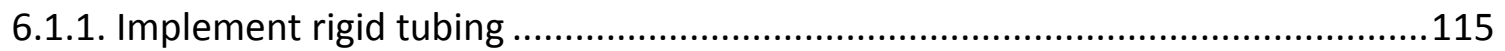

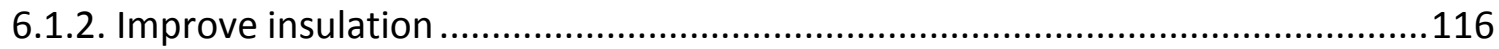

6.1.3. Consider alternative pressure sensors ….......................................................116

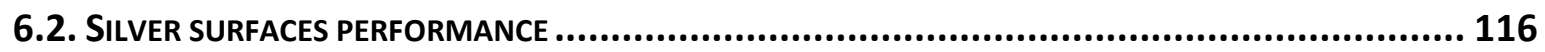

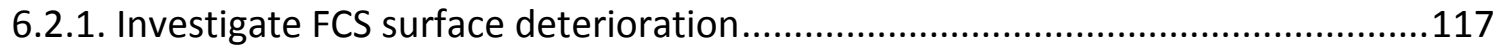

6.2.2. Fabricate and test more FCS surfaces.........................................................117

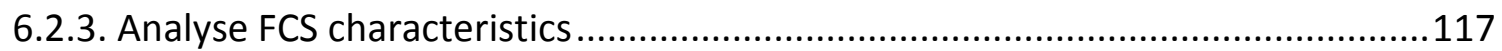

6.2.4. Experiment with freeze cast porous copper......................................................118

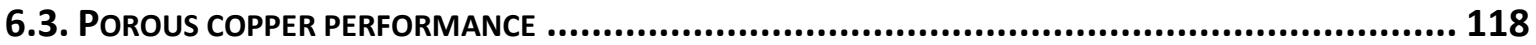

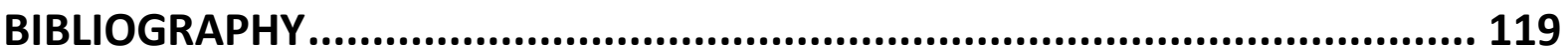




\section{Figures and tables}

\section{Figures}

Figure 1: Conductive heat transfer. $\Delta \mathrm{T}=\mathrm{T} 1-\mathrm{T} 2$

Figure 2: Convective heat transfer, demonstrating free convection. 22

Figure 3: Reynolds' apparatus results showing laminar (a) and turbulent (b) flow. [21] 32

Figure 4: Entrance region (left) and fully developed flow (right). [21] 33

Figure 5: Shape of a fully developed velocity profile for a cylindrical pipe. [8] 34

Figure 6: Shape of a fully developed temperature profile for a cylindrical pipe. [8] 34

Figure 7: Contact angle $\theta$ c. The surface depicted is slightly hydrophilic. 35

Figure 8: Diagram of a regular microchannel. 36

Figure 9: Freeze casting procedure, showing the formation of directionally aligned pores. 40

Figure 10: Heat flux vs excess temperature for various silver concentrations, as well as bare

$\begin{array}{ll}\text { copper. [28] } & 46\end{array}$

Figure 11: Electron microscope pictures of type B (left) and type C (right) pores. 47

Figure 12: Heat flux vs excess temperature for plain copper, FCS surfaces with various silver concentrations, and a sintered silver surface with 40\% silver concentration (Reference 3). [39]

Figure 13: Heat flux vs excess temperature for three consecutive tests. Note the consistently lower heat flux for test 1. [39] 49

Figure 14: Contact angle vs silver concentration in FCS surfaces. [39] 50

Figure 15: Drop lifetime vs contact angle of FCS surfaces. [39] 50

Figure 16: Block diagram of the cooling system. 53

Figure 17: The heater column. The top four holes are used by the heat flux meter. The bottom two holes were unused in this project, but thermocouples could be placed into them to record the temperature between the four heating elements. 55

Figure 18: The four cartridge heaters.

Figure 19: The ring of fibre insulation, with a notch on one side where the thermocouples could be threaded into the heating column. 56

Figure 20: The heat source fully assembled. 56

Figure 21: Heat source cross-section. 
Figure 22: Chamber cross-section.

Figure 23: Cross-section of fully assembled system.

Figure 24: The operation of a diaphragm pump. As the diaphragm expands (top), liquid is pulled into the chamber while the outlet is sealed. Then, as it contracts, the inlet is sealed, and liquid is pushed out via the outlet.

Figure 25: The Favson F312 pump.

Figure 26: Pump free flow characterization.

Figure 27: Heat exchange coil. The entirety of the tubing below the metal plate remains submerged in the cooling tank.

Figure 28: The heat exchanger's thermal resistance with respect to inlet fluid temperature.

Figure 29: The reservoir and bubble trap.

Figure 30: Block diagram of the cooling and sensing systems.

Figure 31: Full heat flux meter equation and diagram of setup. Tn represents the temperature measured by a thermocouple at that point, and dn represents the distance between $\mathrm{Tn}$ and $\operatorname{Tn}+1$.

Figure 32: Thermocouple submerging connector.

Figure 33: The thermocouple calibration results. Note that all eight thermocouples are depicted in this graph, but they are all directly behind one another.

Figure 34: The Omega FTB2003-c hall-effect flow meter.

Figure 35: The flow meter calibration results.

Figure 36: The Omega PX26-030DV differential pressure sensor.

Figure 37: The pressure sensor calibration results.

Figure 38: Circuit diagram of the flow adapter (left) and pressure adapter (right).

Figure 39: The heater control code.

Figure 40: The flow meter code.

Figure 41: The pressure sensor code.

Figure 42: The thermocouple code.

Figure 43: The processing code.

Figure 44: The front end graphing panel during operation.

Figure 45: The front end data panel during operation. 
Figure 46: Surface freezer cross-section.

Figure 47: The temperature profile of the freezing process. Voltage was switched from $-6 \mathrm{~V}$ to $+6 \mathrm{~V}$ after 45 seconds. The periodic raising of the voltage is what results in the strange shape of the temperature curve.

Figure 48: Contact angle measurement device exposed (left) and under light box (right). 88 Figure 49: An example of a contact angle measurement. This sample is exhibiting slight hydrophobicity.

Figure 50: The same sample, after being boiled in DI water. The surface's wettability has changed, and now exhibits hydrophilicity.

Figure 51: An example of a surface exhibiting a contact angle of $0^{\circ}$. The droplet is completely invisible from the perspective of the camera.

Figure 52: Contact angle vs silver mass, before and after boiling.

Figure 53: Contact angle vs PVA mass, before and after boiling.

Figure 54: Versarien porous copper substrate

Figure 55: Pin copper substrate.

Figure 56: 100 Mesh fins substrate coated in porous silver. The mesh fins are still distinguishable in the image.

Figure 57: Thermal interface materials thermal resistance comparison.

Figure 58: Flow rate vs thermal resistance for copper control.

Figure 59: Flow rate and direction vs thermal resistance for copper control.

Figure 60: Flow rate vs thermal resistance for copper control and pin copper.

Figure 61: Infrared picture of surface and wall with system off.

Figure 62: Annotated version of Figure 61. The green box shows the tape that reduces infrared reflection, and the blue box shows the thermal interface between the surface and the substrate.

Figure 63: Infrared picture of surface and wall with system on, showing thermal leakage. 101 Figure 64: Infrared picture of base of heater column with system on, showing thermal leakage.

Figure 65: Corsair H110 liquid cooling system.

Figure 66: Copper control in test system vs Corsair H110 commercial system. 
Figure 67: Thermal resistance vs contact angle at both $1 \mathrm{l} / \mathrm{min}$ and $1.8 \mathrm{l} / \mathrm{min}$ horizontal flow.

Figure 68: Thermal resistance vs contact angle at both $1 \mathrm{l} / \mathrm{min}$ and $1.8 \mathrm{l} / \mathrm{min}$ vertical flow.

Figure 69: Porous copper thickness vs thermal resistance. $0 \mathrm{~mm}$ porous copper is the copper control.

Figure 70: Comparison of thermal resistances under $1 \mathrm{l} / \mathrm{min}$ horizontal flow. Only the lowest thermal resistance of each type of surface is shown.

Figure 71: Comparison of thermal resistances under $1 \mathrm{l} / \mathrm{min}$ vertical flow. Only the lowest thermal resistance of each type of surface is shown.

Figure 72: Comparison of thermal resistances for thick structure tests.

Figure 73: Comparison of hydraulic resistances for thick structure tests. Note the the scale in this case is logorythmic, due to the very high hydraulic resistance of the porous copper. 112

\section{Tables}

Table 1: Comparison of typical thermal resistances for commercial cooling systems. Note that this table is only intended for comparing how the cooling categories can affect the thermal resistance; the thermal resistances of these different systems can fall outside of these ranges.

Table 2: Table of previous research. [38]

Table 3: The pump's free-flow characteristics.

Table 4: The thermocouple calibration values.

Table 5: The flow meter calibration values.

Table 6: The pressure sensor calibration values.

Table 7: Summary table of key results from the test system evaluation.

Table 8: Summary table of key results of porous surfaces.

Table 9: Summary table of key results of thick structures. 


\section{Nomenclature}

\begin{tabular}{|c|c|c|}
\hline Symbol & Description & SI Unit \\
\hline$R e$ & Reynolds number & None \\
\hline$u$ & Fluid velocity & $\mathrm{m} / \mathrm{s}$ \\
\hline$L$ & Characteristic length & $\mathrm{m}$ \\
\hline$v$ & Viscosity & $\mathrm{m}^{2} / \mathrm{s}$ \\
\hline$D_{H}$ & Hydraulic Diameter & $\mathrm{m}$ \\
\hline$A$ & Area & $m^{2}$ \\
\hline$P$ & Perimeter & $\mathrm{m}$ \\
\hline$x$ & Distance & $\mathrm{m}$ \\
\hline$r$ & Radius & $\mathrm{m}$ \\
\hline$q^{\prime \prime}$ & Heat Flux & $\mathrm{W} / \mathrm{m}^{2}$ \\
\hline$k$ & Thermal conductivity & $\mathrm{W} / \mathrm{m} \cdot \mathrm{K}$ \\
\hline$T$ & Temperature & K \\
\hline$l$ & Length & $\mathrm{m}$ \\
\hline$q$ & Power & W \\
\hline$h$ & Convective heat transfer coefficient & $\mathrm{W} / \mathrm{m}^{2} \cdot \mathrm{K}$ \\
\hline$N u$ & Nusselt number & None \\
\hline$R a$ & Rayleigh number & None \\
\hline $\operatorname{Pr}$ & Prandtl number & None \\
\hline$g$ & Gravitational acceleration & $\mathrm{m} / \mathrm{s}^{2}$ \\
\hline$\beta$ & Volumetric thermal expansion coefficient & $1 / K$ \\
\hline$\alpha$ & Thermal diffusivity & $\mathrm{m}^{2} / \mathrm{s}$ \\
\hline$\sigma$ & Stefan-Boltzman constant & $\mathrm{W} / \mathrm{m}^{2} \mathrm{~K}^{4}$ \\
\hline$\varepsilon$ & Emissivity & None \\
\hline$\theta$ & Thermal resistance & K/W \\
\hline$w$ & Width & $\mathrm{m}$ \\
\hline$Z$ & Height & $\mathrm{m}$ \\
\hline$p$ & Pressure & $\mathrm{Pa}$ \\
\hline$F$ & Volumetric flow rate & $1 / \mathrm{s}$ \\
\hline$R_{H}$ & Hydraulic resistance & $\mathrm{Pa} \cdot \mathrm{s} / \mathrm{I}$ \\
\hline
\end{tabular}




\begin{tabular}{|c|c|c|}
\hline$V$ & Voltage & $\mathrm{V}$ \\
\hline$I$ & Current & $\mathrm{A}$ \\
\hline$E$ & Electrical resistance & $\Omega$ \\
\hline$V$ & Volume & $\mathrm{L}$ \\
\hline$f$ & Frequency & $\mathrm{Hz}$ \\
\hline$\theta_{c}$ & Contact angle & $\circ$ \\
\hline$C_{w}$ & Specific heat capacity & $\mathrm{J} / \mathrm{g} \cdot \mathrm{K}$ \\
\hline$\mu$ & Mass flow rate & $\mathrm{g} / \mathrm{s}$ \\
\hline
\end{tabular}




\section{Introduction}

As a natural product of operation, most electronic components produce waste heat, mainly due to the presence of small internal resistances. While small amounts of heat present are typically acceptable, significant amounts can lead to premature deterioration of components, or even component failure. This has become a significant problem in a number of areas, from high end processing units in computers [1], to photovoltaic cells [2], to high-power batteries in electric vehicles [3]. In each of these circumstances, and many more, there is an increasingly large demand for cooling systems that can dissipate larger quantities of waste heat.

Air cooling is a common solution to low amounts of waste heat, but it encounters problems when a large density of heat is generated in one place, as air has a low thermal conductivity [4]. In these cases, active liquid cooling is often an appropriate solution. This involves pumping a liquid coolant over the heat source, which allows the heat to be transferred into the fluid and moved or spread out for dissipation.

In 1981 a landmark paper by Tuckerman and Pease established the capability of microchannels to vastly improve heat transfer in comparison to conventional systems [5]. Since then, research into microchannels has boomed, and the area of interest has expanded from simple parallel channels to a wide variety of possible channel geometries. Porous microstructures are of particular interest, as the processes used to make them can produce microscale channels with relative ease. In particular, they are generated in a semi-random fashion, rather than fabricated using machining or etching.

The aim of this project was to design an active liquid cooling test system suable for testing various surfaces. The objectives under this aim were as follows:

- Assemble an active liquid cooling system

- Integrate test elements for data analysis

- Evaluate the performance of this test system 
An additional aim was to use the designed system to measure the heat transfer capabilities of porous silver surfaces. These surfaces are comparatively easy to fabricate, and the fabrication specifications can be varied to give surfaces with different properties. The objectives under this aim were to:

- Measure the effects of different fabrication specifications on the finished surface's contact angle

- Measure the effects of contact angle on heat transfer in liquid cooling

- Measure the heat transfer properties of some other surfaces for context

This thesis describes the design and evaluation of this integrated cooling system. Relevant background theory, including the fundamentals of heat transfer, the various types of cooling systems, and information regarding microchannels for use in heat transfer, is outlined in Chapter 2. The design and operation of the integrated test system is covered in Chapter 3, including the calibration of the sensing system and the errors associated with these measurements. A comprehensive description of the code that runs the system is also provided. The process used in the production of porous surfaces is described in Chapter 4, together with the procedures involved in creating and characterising these surfaces. Additionally, Chapter 4 contains information regarding the fabrication of any other nonstandard surface used in the project. Chapter 5 gives the experimental results, covering the performance of the integrated test system, and the heat transfer properties of a select few porous surfaces. Finally, Chapter 6 discusses the conclusions of the project, as well as potential future work that can be carried out with this system. 


\section{Background theory}

In this chapter, relevant theory to the design of an active liquid cooling system and use of porous microchannels is detailed. Section 2.1 covers the problem description, explaining the reasons why research into these fields is required. In section 2.2 , the basics of heat transfer are discussed, outlining the mechanisms via which it can occur. Section 2.3 details cooling systems, explaining different types of systems and the benefits and costs of each. A brief explanation of flow dynamics as it relates to liquid cooling systems is given in section 2.4. An introduction to microchannels is included in section 2.5, including the purpose of microchannels, the benefits of regular and porous microchannels, and manufacturing processes for porous microchannels. Section 2.6 covers previous porous microchannel heat sink experiments, ending in a table of the results of those experiments for the purposes of comparison. An overview of previous research on freeze cast silver surfaces is included following this in section 2.7 . Finally, section 2.8 provides a brief summary of the key details of this material.

\subsection{Problem description}

Electronic components are not perfectly efficient, and therefore tend to produce heat as a by-product of operation. In addition to this, every component in a circuit, even the wires, has a small resistance associated with it which will convert a small amount of the electrical power running through it into heat. This heat is not a problem in simple circuits, but as the density of the circuit increases or the power involved gets larger, this heat can pose a significant risk to the integrity of the circuit.

The best example of where this has become a problem in ordinary consumers' lives is highend central processing units (CPUs) and graphics processing units (GPUs). CPUs and GPUs tend to follow Moore's Law, which states that the density of transistors on integrated circuits (ICS) will approximately double every two years [6]. However, the heat density of these ICS increases by a similar amount, and the inability to cool these ICs sufficiently is one of the major factors that is leading to a plateau in performance of modern personal computers (PCs) [1]. 
Another common example is photovoltaic cells. With interest in renewable energy generation continuing to thrive, photovoltaic cells such as solar panels are remaining a subject of interest. However, keeping them cool is an important part of efficient power generation, as the efficiency of photovoltaic cells decreases as their temperature increases [2]. In addition, this cooling method must be efficient, as using too much power would be negating the increase in output from the cell.

High-power batteries for electric vehicles offer a third example of waste heat becoming a problem. The efficiency of these batteries drops significantly if they are outside a certain temperature range [3]. In addition, overheating presents a serious problem. Certain types of batteries, such as lithium-ion batteries, tend to spontaneously combust if they overheat, as a result of the energy stored inside them rapidly releasing [7].

These scenarios, and many more, require the removal of waste heat quickly and effectively. Cooling systems have been the focus of research for several decades, with a major focus on improving their heat transfer capabilities in order to tackle this problem.

\subsection{Heat transfer}

The main focus of this project is improving heat transfer, and a comprehension of what heat transfer is and how it works is essential. While some of the concepts that will be introduced here have a negligible effect overall, it is important to understand why they can be disregarded.

\subsubsection{Heat transfer via conduction}

Conduction is the name given to the heat transfer process that occurs in a stationary medium with a temperature gradient across it. A temperature gradient means that the molecular or atomic structure of the medium has some particles with more energy than others, and as these particles interact with each other, this energy will transfer from the higher energy particles to the lower energy ones. 
This flow of energy, the heat flux $q^{\prime \prime}$, is given by

$$
q^{\prime \prime}=k \frac{\Delta T}{l}
$$

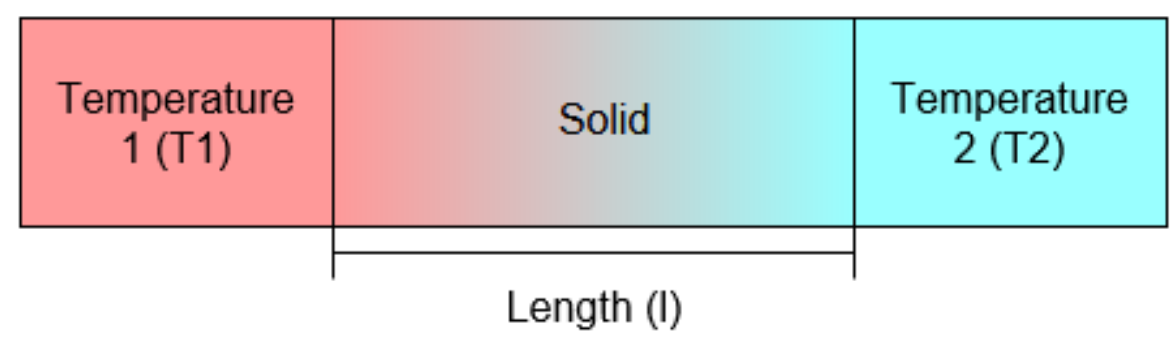

Figure 1: Conductive heat transfer. $\Delta T=T 1-T 2$.

where $\Delta T$ is the temperature differential, $l$ is the distance over which the temperature differential occurs, and $k$ is the thermal conductivity of the materials between the differential [8] [9] [10]. Note that without any outside influence, this heat flux will reduce itself to zero as the energy flow reduces the temperature differential. On the other hand, if there is a constant input power at the hot side, and the cold side can consistently remove the excess energy, this heat flux will be constant, given by

$$
q^{\prime \prime}=\frac{q}{A}
$$

where $q$ is the input power and $A$ is the area over which the heat transfer occurs (tangential to $l$ above) [8] [11] [12].

\subsubsection{Heat transfer via convection}

Convection is the term used to describe heat transfer due to the movement of fluid particles that are carrying heat, rather than the movement of heat between solid particles in conduction. It has two main components: diffusion, where a high concentration of particles carrying the heat slowly spread themselves evenly into the fluid, and advection, where the particles flow together in bulk. Each of these can occur on their own, or jointly.

Convective flow is a commonly recognised natural phenomenon. As the particles on a hot surface heat up, they spread out, lowering their density. If the hot surface is beneath them, 
this change in density will cause them to rise off the surface, and for the cooler, less dense fluid to sink, flowing in to replace the rising fluid. This flow is also known as free convection.

However, the fluid flow does not need to come from free convection. A continuous fluid flow across a hot surface will still cause the surface to transfer heat via convection, as the heated particles are removed by the flow and replaced with new, cooler particles. This type of convection is known as forced convection. Typically, free convection will still take place in this scenario, but it would not be the sole driving force. Because of this, introducing forced convection allows a system to have greater heat transfer than if it were exclusively transferring via free convection.

The heat flux associated with convection is given by

$$
q^{\prime \prime}=h\left(T_{s}-T_{\infty}\right)
$$

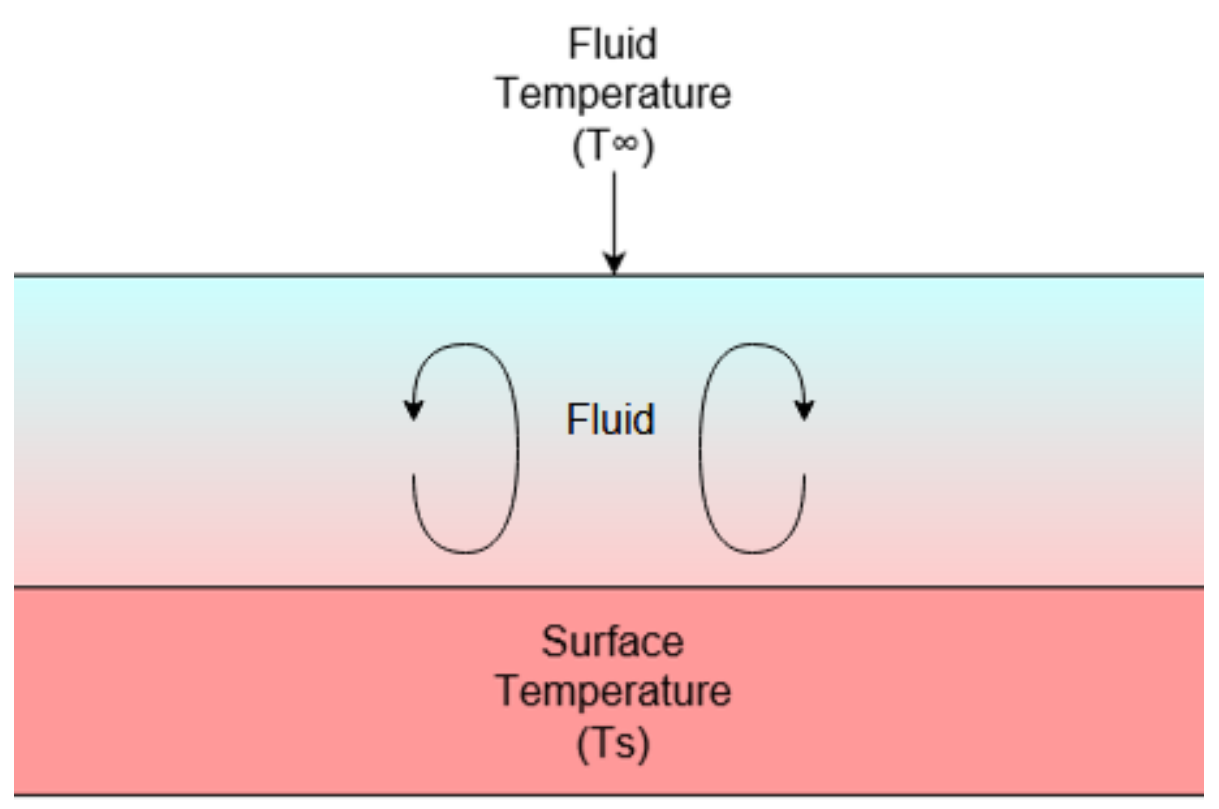

Figure 2: Convective heat transfer, demonstrating free convection.

where $T_{S}$ is the temperature of the surface, $T_{\infty}$ is the temperature of the fluid, and $h$ is the convective heat transfer coefficient [8] [9] [11]. This equation is somewhat misleading, as it looks simple, but $h$ is dependent on a large number of variables defining the exact interactions between the surface and the fluid, and so it can be difficult to calculate. 
However, the convective heat transfer coefficient can be expressed using the Nusselt number $N u_{L}$ as given by

$$
N u_{L}=\frac{h L}{k}
$$

where $L$ is the characteristic length [8] [10]. For the general case $L$ is defined as the hydraulic diameter $D_{H}$ given by

$$
D_{H}=\frac{4 A_{c}}{P_{c}}
$$

where $A_{c}$ is the cross-sectional area of the pipe, and $P_{c}$ is the cross-sectional perimeter [11]. The hydraulic diameter is used to approximate a tube or channel as a round tube. Note that for circular pipes $D_{H}$ is just the diameter of the pipe.

For free convection, the Nusselt number is a function of the Rayleigh number $R a$ and the Prandt number $\mathrm{Pr}$. These are dimensionless numbers that define the relationship between buoyancy and viscosity in a fluid and viscosity and thermal diffusion in a fluid, respectively.

These numbers are given by

$$
R a_{L}=\frac{g \beta\left(T_{s}-T_{\infty}\right) \rho c_{p} L^{3}}{v k}
$$

and

$$
\operatorname{Pr}=\frac{v}{\alpha}
$$

where $g$ is the gravitational acceleration, $\beta$ is the volumetric thermal expansion coefficient, $v$ is the kinematic viscosity of the fluid, and $\alpha$ is the thermal diffusivity of the fluid [8]. The Prandtl number describes the ratio of momentum and thermal diffusivities of the fluid. The Rayleigh number describes the ratio of buoyancy and viscosity forces multiplied by the Prandtl number. 
The function that defines the Nusselt number with respect to these numbers in free convection varies based on geometry and other boundary conditions, and a number of specific cases have corresponding empirical correlations. However, general patterns exist in the effect that the components of the Rayleigh and Prandtl numbers have on the Nusselt number, and more importantly, how they affect the convective heat transfer coefficient.

Increasing the gravitational acceleration or the thermal expansion coefficient will increase the buoyancy force which drives advection, logically increasing the convective heat transfer coefficient. Increasing the temperature difference between the surface and the surrounding fluid or the thermal conductivity of the fluid will increase the conduction of heat into the fluid, also increasing the convective heat transfer coefficient. Increasing the volumetric heat capacity of the fluid will cause more energy to be transferred between the surface and the fluid for a given temperature difference, so this too will increase the convective heat transfer coefficient.

In contrast, increasing the viscosity of the fluid will increase the viscous forces acting against flow. This will result in a decrease in the rate of fluid flow, and while it will also increase the momentum diffusivity, this will have an overall negative impact on the convective heat transfer coefficient.

Finally, increasing the characteristic length will increase the ratio of cross-sectional area to perimeter within the heat transfer medium. Expanding to the third dimension, this is an increase in the ratio of volume to surface area. This means that there is comparatively less of the fluid being heated by the heat source, so the convective heat transfer coefficient will be decreased as a result.

For forced convection, the Nusselt number is instead a function of the Reynolds number $R e$ and the Prandtl number. The Reynolds number is another dimensionless number. This describes the relationship between the force of motion and the viscosity in the fluid. It is defined by

$$
R e_{L}=\frac{u L}{v}
$$


where $u$ is the fluid's velocity. The change from the Raleigh number to the Reynolds number describes the change in the dominant form of motion in the fluid. Again, this function varies based on certain boundary conditions, but general rules exist that relate the components of the Reynolds number and the Prandtl number to the convective heat transfer coefficient.

Increasing fluid velocity will obviously increase advection, so this increases the convective heat transfer coefficient. As with free convection, increasing the volumetric heat capacity will increase the heat energy transferred into the fluid, so that too will increase the convective heat transfer coefficient.

An increase in viscosity will result in an increase to the viscous forces acting against flow. As with free convection, this will have a positive effect on the momentum diffusivity but an overall negative effect on the convective heat transfer coefficient. Increasing the characteristic length will also have the same negative effect as it does in free convection.

These forms give an idea of what effects different variables will have on the convective heat transfer coefficient. However, it should be noted that special cases exist that can cause certain variables to effectively have no impact on the convective heat transfer coefficient. Of particular note is the special case of a fully developed tube.

When flowing over a surface or through a tube, fluid will form a temperature profile. The temperature of the fluid at the surface will be approximately equal to the temperature of the surface itself, and the temperature will decrease as the fluid gets further away from the surface. As the fluid travels further along the surface, this temperature front will stretch over the fluid's entire height until it encompasses the entire channel. The area covered before this point is called the thermal entrance region, while from this point on the temperature profile is considered fully developed [8] [11] [13]. If the flow is laminar and fully developed, the Nusselt number will have a constant value, determined by the geometry of the channel and the particular nature of the heat source. As the convective heat transfer coefficient is related to the Nusselt number only by the thermal conductivity and characteristic length, these are the only two values that will have an effect on the convective heat transfer coefficient for a system that is laminar and fully developed [5] [8]. 


\subsubsection{Heat transfer via radiation}

Finally, some heat transfer occurs via the process of radiation. Any surface with a finite temperature will emit some heat energy as electromagnetic waves due to changes in electron configurations in the atoms constituting the substance. Because this is electromagnetic radiation, no medium is required, as it is actually more efficient in a vacuum. However, this process can also occur in reverse, with electromagnetic waves being absorbed by the surface, heating it up. The heat flux via radiation is given by

$$
q^{\prime \prime}=\varepsilon \sigma\left(T_{s}^{4}-T_{\text {sur }}^{4}\right)
$$

where $T_{S}$ is the temperature of the surface, $T_{\text {sur }}$ is the temperature of the surroundings, $\sigma$ is the Stefan-Boltzman constant $\left(\sigma=5.67 \times 10^{-8} \mathrm{~W} / \mathrm{m}^{2} \cdot K^{4}\right)$, and $\varepsilon$ is the emissivity of the surface $(0 \leq \varepsilon \leq 1)$ [8] [9] [11].

It should be noted that, in most systems, these heat transfer processes will apply simultaneously. This means that the total heat flux from a surface will be given by

$$
q^{\prime \prime}=q^{\prime \prime}{ }_{\text {conv }}+q^{\prime \prime}{ }_{\text {rad }}=h\left(T_{s}-T_{\infty}\right)+\varepsilon \sigma\left(T_{s}^{4}-T_{\text {sur }}^{4}\right)
$$

where $q^{\prime \prime}{ }_{\text {conv }}$ is the convective heat flux and $q^{\prime \prime}{ }_{\text {rad }}$ is the radiative heat flux. However, for reasonable temperature differences, the amount of heat lost via radiation is small in comparison to the amount lost via convection, and it can often be ignored as a result.

\subsection{Heat transfer in cooling systems}

The purpose of a cooling system is to remove heat energy from a heat source. A cooling system's performance is measured by its thermal resistance $\theta$ given by

$$
\theta=\frac{\Delta T}{q}
$$


where $\Delta T$ is the change in temperature between the surface and the coolant and $q$ is the power transferred into the coolant [8] [10] [11]. Because this is a resistance, cooling systems perform better if they have a lower $\theta$. That is, for a given temperature difference, the dissipated power should be maximized.

For conduction, $\theta$ is given by

$$
\theta=\frac{l}{k A}
$$

where $A$ is the area over which the heat transfer occurs, $l$ is the distance over which the temperature differential occurs (tangential to $A$ ), and $k$ is the thermal conductivity of the materials between the differential. This means that to minimize $\theta$, the area of conduction and thermal conductivity should be maximized and the distance tangential to the area of conduction should be minimized.

For convection, $\theta$ is given by

$$
\theta=\frac{1}{h A}
$$

where $h$ is the convective heat transfer coefficient. Similar to conduction, $\theta$ is minimized in this case when the area and convective heat transfer coefficient are maximized.

For radiation, $\theta$ is given by

$$
\theta=\frac{1}{\varepsilon \sigma\left(T_{s}+T_{\text {sur }}\right)\left(T_{s}^{2}+T_{\text {sur }}^{2}\right) A}
$$

In order to minimize $\theta$, the surface area, emissivity and operating temperatures should be maximized.

A cooling system with multiple heat transfer processes in series (where the output of one process feeds into the input of the next) will have a thermal resistance equal to the sum of 
each individual process's thermal resistance. Multiple heat transfer processes in parallel (where the inputs of multiple processes come from one output) will have a thermal resistance given by

$$
\frac{1}{\theta_{T}}=\frac{1}{\theta_{1}}+\frac{1}{\theta_{2}}+\frac{1}{\theta_{3}}+\cdots
$$

where $\theta_{T}$ is the total resistance of the system, and $\theta_{1}, \theta_{2}$, etc. are the individual thermal resistances [11]. This equation means that $\theta_{T}$ will always be less than or equal to the smallest resistance present.

It should be noted that it is impossible to create a cooling system that operates purely by conductive processes. Without moving the heated particles away, like convection, or radiating the energy away, the conductor will eventually reach the temperature of the heat source and cooling will cease. Similarly, a cooling system with near-exclusively radiative cooling will only exist in a near-perfect vacuum, because any fluid the system is immersed in, including air, will lead to convective cooling occurring. Furthermore, the radiative resistance will typically be larger than the convective resistance, and will be in parallel to it, which means its effects on the total thermal resistance will be low.

\subsubsection{Types of cooling systems}

Cooling systems can broadly be broken into two categories based on the dominant form of convection (passive and active) [14], two categories based on the cycle of fluid (open-loop and closed-loop) [15], and three categories based on the state of matter of the working fluid (air, liquid, and two-phase) [16].

Passive cooling systems are dominated by free convection, while active cooling systems are dominated by forced convection. Typically, active cooling systems are better at cooling than passive cooling systems due to increased advection, but they require a pump, fan, or other mechanism to keep the fluid flowing, which can increase cost. They also produce waste noise, which is something certain applications would prefer to minimize. 
Open-loop systems remove their fluid once it has become heated, replacing it with new fluid. Closed-loop systems opt instead to cool that liquid and reuse it. This requires a heat exchanger, which will both increase the cost and introduce a second heat transfer point which must be considered, as exceeding the heat exchanger's capacity will lead to the recycled fluid increasing in temperature. Open-loop systems, on the other hand, require the constant input of new fluid which, under most circumstances, will increase the cost.

Air and liquid cooling systems use gases and liquids as their coolants, respectively. In general, liquid cooling is more effective than air cooling, as liquids tend to have a much higher thermal conductivity than gases. For example, water's thermal conductivity is about $0.598 \mathrm{~W} / \mathrm{m} \cdot \mathrm{K}$ [17], while air has a thermal conductivity of about $0.024 \mathrm{~W} / \mathrm{m} \cdot \mathrm{K}$ [18]. However, the vast majority of air-cooling systems use the ambient air of the room, which means they are typically significantly cheaper than liquid-cooling systems because they do not require the purchase of coolant. This also means that open-loop air-cooling systems do not have the cost issues of normal open-loop systems, and because of this, closed-loop air cooling systems are impractical.

Two-phase cooling systems use a phase change (that is, a change from one state of matter to another) to facilitate cooling. Almost always, this phase change will be between liquid and gas. Two-phase systems are used because the heat energy required to perform a phase change, called the latent heat, is much larger than the amount of energy to increase the same substance's temperature, called the specific heat. In the case of water, the specific heat is $4.18 \mathrm{j} / \mathrm{g}$ [17] [18], while the latent heat is $2260 \mathrm{j} / \mathrm{g}$ [17] [18]. This means that the thermal resistance is significantly lower if the fluid is undergoing a phase change. However, two-phase systems require the coolant to be at the phase boundary, which means that the heat source must be very hot, the coolant must have a low evaporation temperature, or the fluid must be under pressure to lower the evaporation temperature.

In practice, only a handful of combinations are actually commonly used, as the majority of combinations are impractical outside of niche applications. The few that are used offer a relatively linear trade-off between cooling efficiency and cost. 
For low power heat sources, passive air cooling is normally sufficient. This consists of a simple heat sink, which is a structure with a high thermal conductivity, large surface area and low distance from the heat source to the surface. This minimizes the heat sink's thermal resistance due to an increased heat transfer area. This can be further improved by adding a fan to facilitate fluid flow, turning it into an active cooling system.

If an air cooling system is not sufficient to cool a heat source, the next best options are active closed-loop liquid cooling systems and heat pipes, depending on the space available for the system.

Heat pipes are passive closed-loop two-phase systems, but their thermal resistance is typically worse than liquid cooling systems. This is because they lose some efficiency in order to massively scale down. Heat pipes themselves take up very little space, although like all closedloop systems they still need a heat exchanger, which will take up considerable space. However, they can be used to spread or relocate the heat they are cooling so that a larger open-loop cooling system can dissipate the heat.

Active closed-loop cooling systems achieve a similar goal, relocating heat to a position that can accommodate a reasonably large heat exchanger. However, they are considerably bulkier than heat pipes, and require a pump to drive fluid flow, which further adds to the space required. This increase in size comes with an increase in heat transfer capabilities, and so liquid cooling systems are used in scenarios where thermal resistance is a more crucial variable to minimize than size.

\begin{tabular}{|c|c|c|c|}
\hline Cooling system type & Air cooling & Heat pipe & $\begin{array}{c}\text { Closed loop } \\
\text { liquid cooling }\end{array}$ \\
\hline $\begin{array}{c}\text { Typical thermal resistance } \\
\text { range }\end{array}$ & $0.40-0.46$ & $0.36-0.43$ & $0.28-0.34$ \\
\hline
\end{tabular}

Table 1: Comparison of typical thermal resistances for commercial cooling systems. Note that this table is only intended for comparing how the cooling categories can affect the thermal resistance; the thermal resistances of these different systems can fall outside of these ranges. [19] 


\subsection{Flow dynamics}

As this project uses active liquid cooling, it is important to have a cursory understanding of flow dynamics. Flow dynamics is about how the motion of different areas of the fluid change due to their flow conditions, and the flow dynamics discussed below details this for the specific case of fluid flowing through a pipe.

\subsubsection{Laminar and turbulent flow}

Firstly, it is important to understand laminar and turbulent flow. In 1883, Osborne Reynolds distinguished two different types of fluid flow, which occur based on the magnitude of the flow rate, using an apparatus that injected a dye stream into a flowing fluid. A diagram depicting the apparatus used is provided in Figure 3 [11]. For flow rates considered "small enough", the dye stream remained mainly linear, with only a small amount of diffusion into the water at its edges. At intermediary flow rates, the dye stream would move through the fluid, with irregularity in its motion, and would diffuse more easily. Finally, at flow rates sufficiently "large enough", the dye stream would rapidly diffuse into the fluid, spreading in a random fashion as it did so. These flow types were coined laminar, transitional, and turbulent flow, respectively, and are depicted in Figure 3.

However, the boundaries of these flow types can change depending on certain variables, such as the viscosity of the fluid in question. To resolve this, the Reynolds number is used. As mentioned in section 2.2.2, the Reynolds is a unitless number that relates the ratio of inertial forces to viscous forces in a fluid. For flow with a Reynolds number less than around 21002300 , the flow is laminar, while flow with a Reynolds number exceeding approximately 4000 is turbulent [8] [11] [20]. Flow between these values is considered transitional flow. 


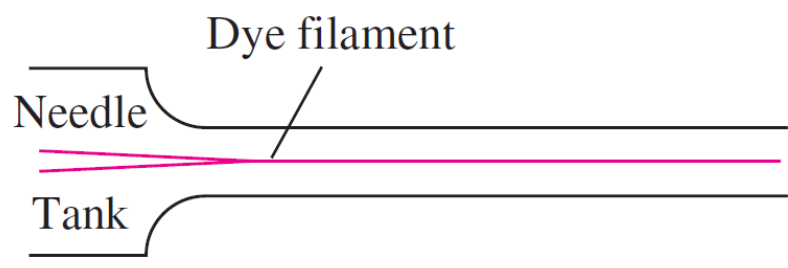

(a)

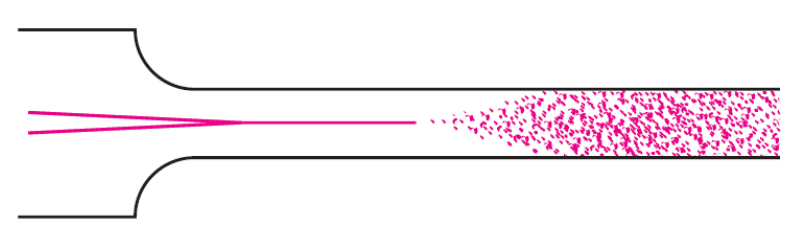

(b)

Figure 3: Reynolds' apparatus results showing laminar (a) and turbulent (b) flow. [21]

\subsubsection{Regions of flow}

Fluid flowing through a pipe will naturally stick to the inner wall due to viscous effects. Because of this, the velocity of the fluid will be different based on its distance from the centre of the pipe. As the fluid enters the pipe, only the very edges of it will be slowed down significantly, but after a certain length the viscous effect will have slowed the entire cross section of fluid. Before this point, the fluid is in the hydrodynamic entrance region of flow. Over this region, the area of fluid being slowed, known as the hydrodynamic entrance region, will steadily increase until it encompasses the entire flow front, at which point the flow is considered fully developed [8] [11] [13]. A diagram depicting these regions is provided in Figure 4 [21]. 


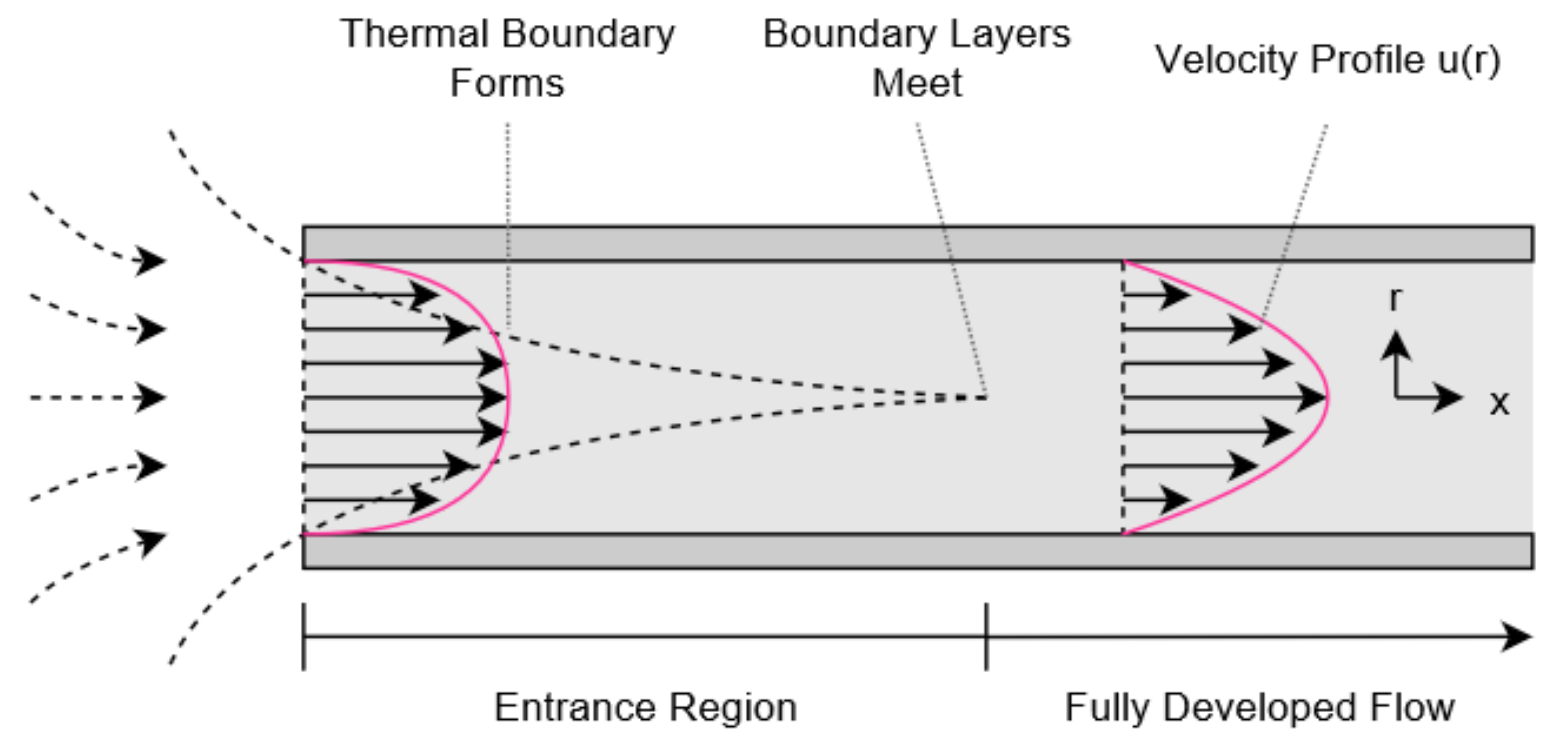

Figure 4: Entrance region (left) and fully developed flow (right). [21]

For laminar flow, the length of the hydrodynamic entrance region $x_{f d, h}$ may be expressed by

$$
\frac{x_{f d, h}}{L} \leq 0.05 R e_{L}
$$

while for turbulent flow it may be expressed by

$$
10 \leq \frac{x_{f d, h}}{L} \leq 60
$$

and the velocity profile $u(r)$ for fully developed flow in a cylindrical pipe is given by

$$
u(r)=u_{c}\left[1-\left(\frac{2 r}{L}\right)^{2}\right]
$$

where $r$ is the distance from the centre of the pipe and $u_{c}$ is the velocity of the fluid at the centre of the pipe. 


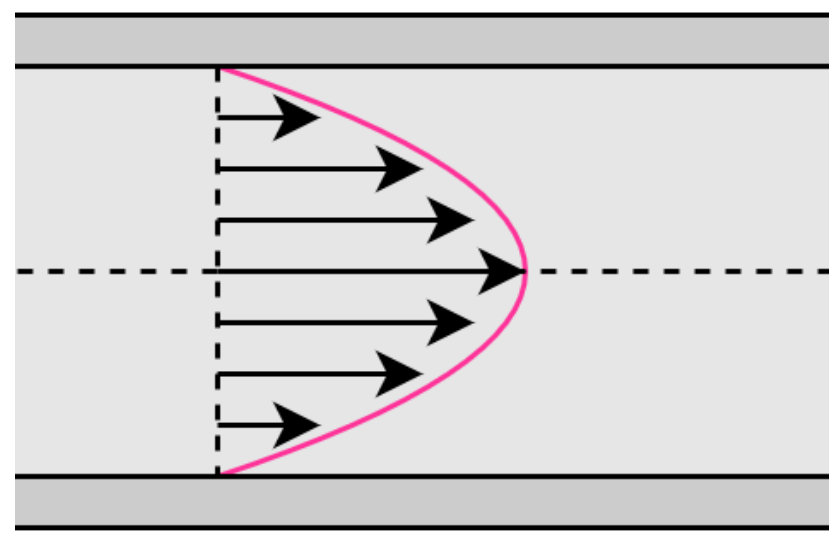

Figure 5: Shape of a fully developed velocity profile for a cylindrical pipe. [8]

Now recall the thermal entrance region from section 2.2.2. These two profiles are analogous, and as such, a similar thermal entry length $x_{f d, t}$ exists. For laminar flow, this is expressed by

$$
\frac{x_{f d, t}}{L} \leq 0.05 R e_{L} \operatorname{Pr}
$$

while for turbulent flow it may be expressed by

$$
\frac{x_{f d, t}}{L}=10
$$

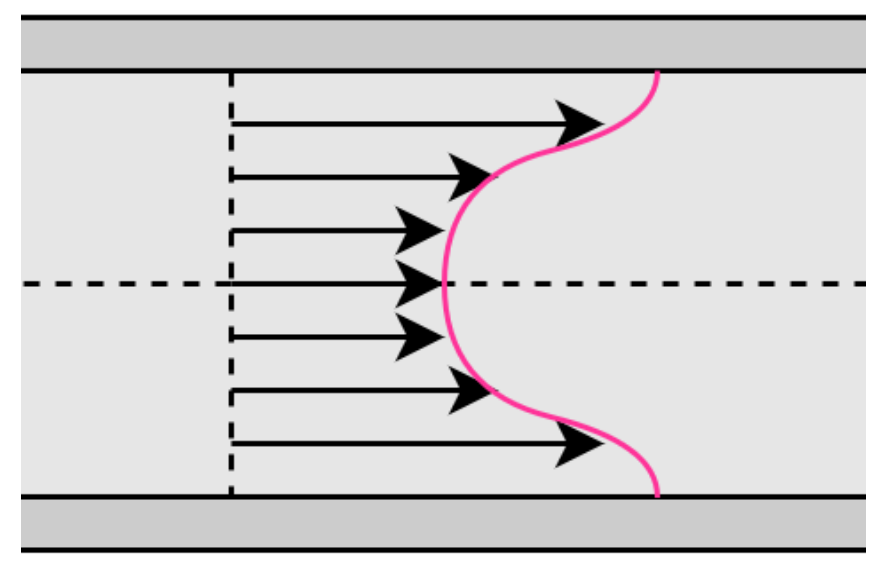

Figure 6: Shape of a fully developed temperature profile for a cylindrical pipe. [8]

\subsubsection{Hydraulic resistance}

Due to friction and viscous forces acting against the force of flow, the fluid travelling through a pipe will experience a resistance. This resistance is called the hydraulic resistance, and is given by 


$$
R_{h}=\frac{\Delta \mathcal{P}}{F}
$$

where $\Delta \mathcal{P}$ is the pressure differential across the pipe, and $F$ is the flow rate through it. As a maximum inlet pressure will exist for a given system, this resistance provides a maximum flow rate and in turn a maximum fluid velocity for said system.

\subsubsection{Wettability}

Wettability is a measure of the balance between the adhesive force of the liquid on the surface, and the cohesive force holding the liquid together. One statistic that can be used to communicate the wettability of a surface is that surface's contact angle. Contact angle details the angle formed at the edge of a droplet of liquid on the surface and is always between the bounds $0^{\circ} \leq \theta_{c} \leq 180^{\circ}$. A surface with a contact angle between $0^{\circ} \leq \theta_{c} \leq 90^{\circ}$ is considered to be hydrophilic, and a surface with a contact angle between $90^{\circ} \leq \theta_{c} \leq 180^{\circ}$ is considered to be hydrophobic [22].

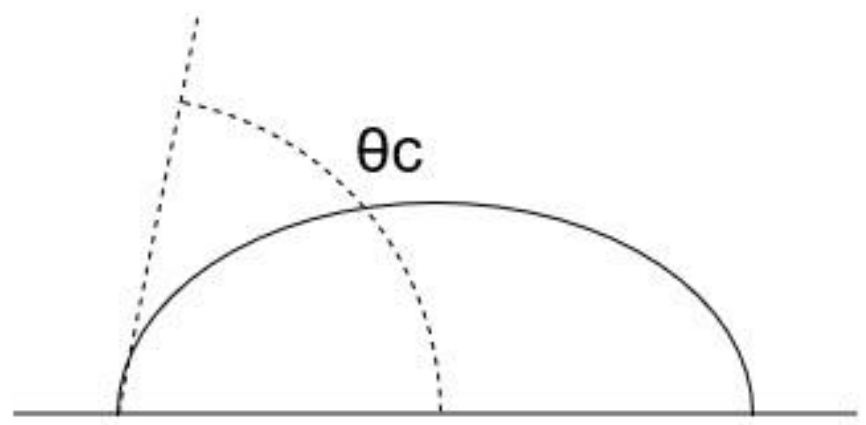

Figure 7: Contact angle $\theta_{c}$. The surface depicted is slightly hydrophilic.

\subsection{The use of microchannels in liquid cooling systems}

A liquid cooling system will typically have at least two thermal resistances in series to consider: the conductive resistance of the heat $\operatorname{sink} \theta_{\text {cond }}$ and the convective resistance of the fluid $\theta_{\text {conv }}$. As these are in series, the best method of decreasing the total thermal resistance is to reduce each as much as possible. A given heat source will typically have a set area of conduction, so minimizing the length of conduction and maximizing the solid's thermal conductivity are the best methods for minimizing $\theta_{\text {cond }}$. To reduce $\theta_{\text {conv }}$, the surface area in contact with the fluid must be maximized, as must the convective heat transfer coefficient. 
As discussed in section 2.2.2, the most effective methods of increasing the convective heat transfer coefficient for laminar flow in a pipe are increasing the thermal conductivity of the fluid and decreasing the characteristic length. Assuming the coolant being used has a sufficiently high thermal conductivity, implementing microchannels is the easiest way to reduce this resistance.

Microchannels are, as the name implies, microscale structures on the thermal surface. These decrease the characteristic length, increasing the convective heat transfer coefficient as a result. A diagram of a classic microchannel surface, including channel width $w_{c}$, wall width $w_{w}$ and wall height $z$, is provided in Figure 8 .

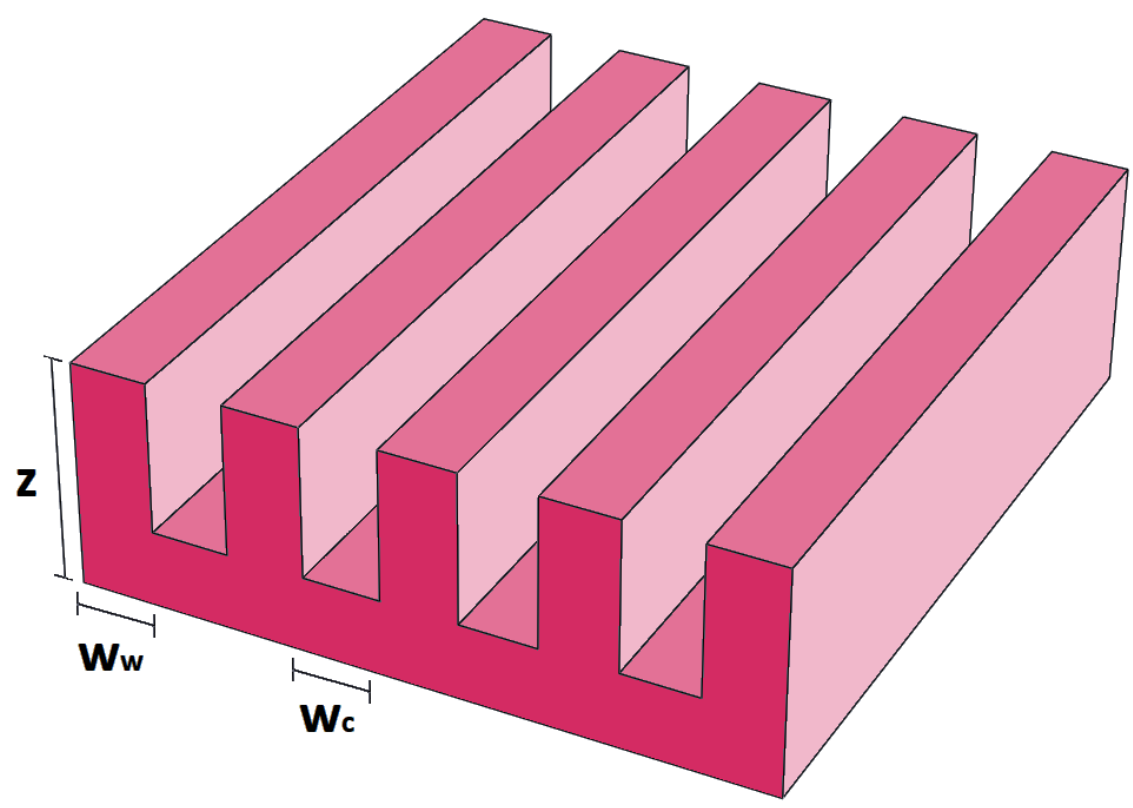

Figure 8: Diagram of a regular microchannel.

There are, however, costs associated with microchannels. To decrease the characteristic length, the wall and channel widths $w_{w}$ and $w_{c}$ must be made smaller while the wall height $z$ must be made larger, but this will increase the thermal resistance of conduction up these channel walls. As such, for a given cross-sectional surface area, there will be some optimum point where the decrease in $\theta_{\text {conv }}$ will no longer outweigh the increase in $\theta_{\text {cond }}$ on the channel walls. However, it is very difficult to find this optimum point due to its dependence on individual characteristics of the system and the requirement to optimize three separate 
variables simultaneously. In practice, reducing the characteristic length is typically a good choice.

In addition, as characteristic length approaches zero, the hydraulic resistance of the channel increases, limiting flow rate and increasing pressure at the inlet. While this can be fixed by increasing the capabilities of the pump and the pressure capabilities of the pipes connecting the system, it means that there will be a fundamental limit to the amount that the characteristic length can be reduced for a given system.

In order to discuss microchannels further, they will be classified into two separate categories: regular and porous. Regular microchannels refer to microchannels with a precise geometry, such as the classical microchannel surface depicted in Figure 8, while porous microchannels refer to microchannels which have imprecise geometry, such as metal foams.

\subsubsection{Regular microchannels}

Regular microchannels were initially proposed in Tuckerman and Pease's ground-breaking paper: High-performance heat sinking for VLSI [5]. They are manufactured via accurate processes, such as machining or mask etching, and thus have a precise geometry. The simplest form of regular microchannels is a finned prism, whose cross-section is depicted in Figure 8. However, more complicated forms also exist, with the most common variation being pin fins. Pin fins have separations on multiple axes, making the fins look like pins sticking out from the substrate, hence the name. Pin fins are useful in free convection systems but have little application when forced convection is applied in parallel to the pins, as the extra surface area added between the pins will have little flow through it. Most other regular microchannels will have a similar structure to one of the above, with slight variations for particular applications.

\subsubsection{Porous microchannels}

In contrast to regular microchannels, porous microchannels are generated using inaccurate processes such as dispersal, and thus have an imprecise geometry. This does not mean that the geometry is unpredictable; general statements can be made about the surface, with the most important ones being porosity, average cell size, and cell variance. However, while two 
regular microchannels may appear identical when compared, two porous microchannels with the same surface characteristics most likely will not.

There are two types of porous media: closed-pore and open-pore [23]. Closed-pore media take the form of individual cavities suspended in a solid substrate, with no connection between the cavities. Open-pore media, by contrast, have connections between the pores. For the purposes of microchannels, closed-pores are undesirable for two reasons. Firstly, the fluid can only flow over the outer surface of a closed-pore system, as opposed to through it like with open-pores or regular microchannels, vastly increasing hydraulic resistance. Secondly, as long as the porous material has a higher thermal conductivity than the trapped air inside the closed pores, those pores will give the medium a lower thermal conductivity than an equivalently thick solid block of material, without contributing any benefit to convection.

Open-pore porous microchannels also have benefits over regular microchannels. Most importantly, the interconnections between pores encourage the fluid to mix more than with regular channels, which increases the effective thermal conductivity, thus increasing the heat transfer and reducing the thermal resistance [24].

\subsubsection{Fabricating microchannels}

It should be noted that regular and porous microchannels have significantly different fabrication processes. Regular microchannels require high precision in the fabrication process [25], while porous microchannels do not. However, porous microchannel fabrication will typically require much higher temperatures or pressures than regular microchannels, due to the use of sintering or liquid metal to form the porous substrate. The following section outlines the methods of fabricating open-pore microchannels that are present either in previous experiments, or in this project.

\subsubsection{Fabricating open-pore microchannels}

A simplistic method of fabricating a porous substrate is to compact a metal powder and then sinter it [26]. This creates a three-dimensional lattice of metal with air gaps between. Certain metals, such as aluminium, are difficult to sinter because they naturally coat themselves in an 
oxide layer, but this can be overcome by breaking the oxide layer during the sintering process, or by introducing sintering aids.

To further improve this method, a "lost mass" process can be used. This involves suspending the metal powder in a filler substance, often a solvent, and a space holder. The space holder must be a substance that can be removed from the system without damaging the established structure [27]. This is then sintered or compacted, before the space holder is removed, leaving a porous sponge of metal powder and filler.

Freeze casting is a special form of lost mass, which can be used to make pores with a high degree of alignment [28]. The freeze casting process suspends the metal particles in a solvent solution, typically water [29]. Additional substances may be included to encourage dispersal and binding. This solution is then frozen directionally, encouraging the formation of ice crystals, and these crystals push the impurities in the solution aside, trapping them between the crystals. Removing the crystals via sublimation leaves a delicate porous lattice. Annealing this structure then removes the binders and sinters the particles to strengthen the structure. In addition to its ability to generate highly aligned pores, freeze casting is of interest because it is cheap in comparison to other porous microstructure fabrication processes. 


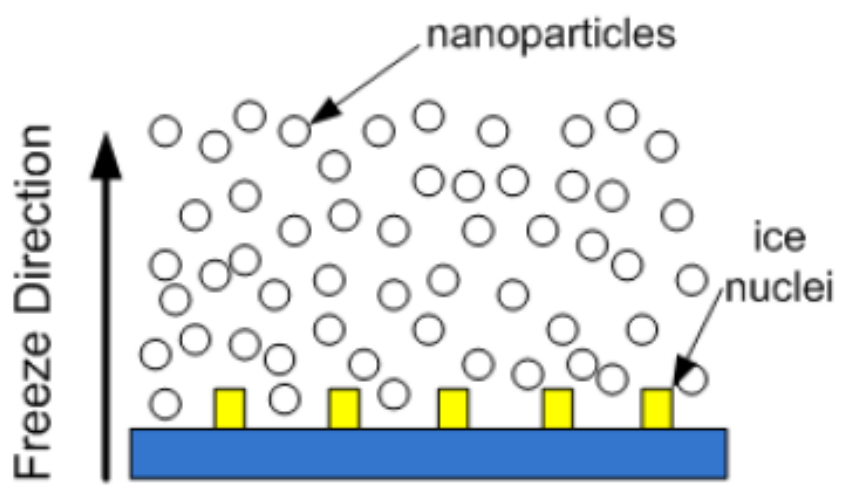

(a)

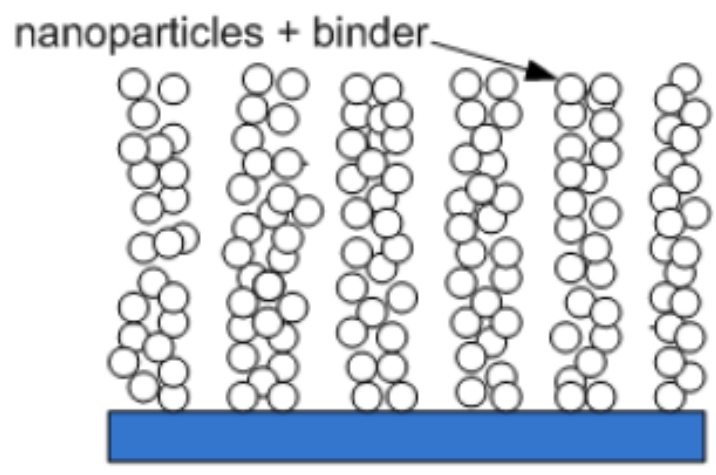

(c)

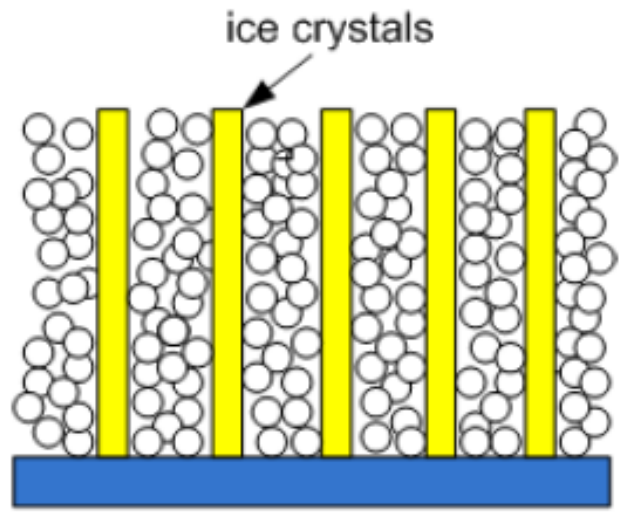

(b)

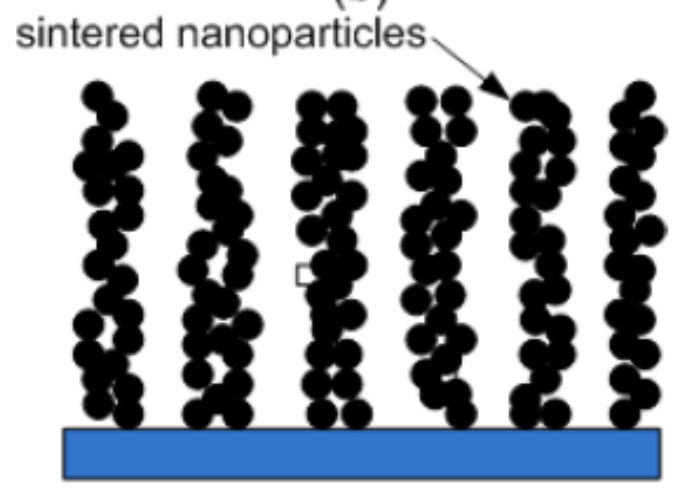

(d)

Figure 9: Freeze casting procedure, showing the formation of directionally aligned pores.

\subsection{Literature review of porous microchannels}

There have been a large number of experiments on liquid cooling systems and porous microchannels over the past few decades. This section lists a few specific examples, describing the setup and key results of the experiments, and compiles a comparison table of notable details of these experiments.

\subsubsection{Details of research}

\subsubsection{Chen et al: Two-phase film heating in bi-dispersed sintered copper}

This experiment investigated the boiling characteristics of mono- and b-dispersed sintered copper particles [30]. Two types of mono-dispersed particles were used, at $80 \mu \mathrm{m}$ and $800 \mu \mathrm{m}$, while three bi-dispersed particle groups were used, with 80/200 $\mu \mathrm{m}, 80 / 400 \mu \mathrm{m}$ and $80 / 800 \mu \mathrm{m}$ particles each. These particles were packed into a channel $30 \mathrm{~mm}$ wide, $10 \mathrm{~mm}$ tall and $50 \mathrm{~mm}$ long. Distilled water was used as the coolant and was heated to aid phasechange via an electronic heater. The test section was heated with a film heater, acting as the 
heat source. The experiment monitored the water temperature at various points, as well as its flow rate, and the pressure at the inlet and outlet of the test section.

The results showed a significantly lower permeability for the $80 \mu \mathrm{m}$ mono-dispersed particles than any of the other samples, and that the bi-dispersed particles permeability approximated the permeability of the larger particles present. These permeability results agreed with the pressure drop results, which showed a significantly higher drop over the $80 \mu \mathrm{m}$ monodispersed particles, around $12 \mathrm{kPa}$, than the other samples, at $\sim 1.3 \mathrm{kPa}$. Conversely, the $800 \mu \mathrm{m}$ mono-dispersed particles had a lower heat transfer coefficient than any of the other samples. This implies that bi-dispersed particle samples can be useful in finding a middle ground between a given pressure drop and heat transfer coefficient.

\subsubsection{Jiang et al: Hot/cold water system with sintered copper particles}

This experiment analysed the heat transfer and pressure drop characteristics of traditional micro-channels and porous microchannels [31]. The porous microchannel being analysed was formed of sintered copper particles with an average diameter of $0.272 \mathrm{~mm}$, sandwiched between copper plates with a separation of around $0.6 \mathrm{~mm}$. Water was used as the working fluid, and the heat source was supplied by hot water running though microchannels perpendicular to the coolant, stacked on top of one another in an alternating pattern. The experiment monitored inlet and outlet water temperatures, flow rates, and pressures.

The results showed that the porous microchannels had a much higher heat transfer coefficient than the traditional microchannels, with $86.3 \mathrm{MW} /\left(\mathrm{m}^{3} \mathrm{~K}\right)$ when both fluids were flowing at $1.06 \mathrm{~m} / \mathrm{s}$ compared to $\sim 12 \mathrm{MW} /\left(\mathrm{m}^{3} \mathrm{~K}\right)$ for the same fluid velocity. However, it also had a significantly higher pressure drop, reaching 4.66 bar compared to the microchannels' $\sim 0.2$ bar at the same fluid velocity.

\subsubsection{Boomsma et al: Liquid cooled heater with compressed aluminium foam}

This experiment investigated the hydraulic and thermal performance of aluminium foams in heat exchangers [32]. The aluminium foam was open-celled, with approximately $2.3 \mathrm{~mm}$ diameter pores. These foams were then compressed, to increase their surface area to volume ratio. The foams were cut to fill a channel $40 \mathrm{~mm}$ wide and long, and $2 \mathrm{~mm}$ tall. Water was 
used as the coolant, and a heater and heat-spreader were used as the heat source. The experiment monitored the temperature of the coolant at various points, the temperature of the heater and heat spreader, the power supplied to the heater, the flow rate, and the pressure drop across the heat exchanger.

The results showed that as the volume-to-surface-area ratio decreased, the pressure drop increased, with the largest maximum pressure differentials being as high as 3 bar, three times the lowest maximum pressure differential. It was also found that, considering the pressure increase, the best performance was from the surface 92-06, which had a large $M$ value and low initial porosity. This foam had a thermal resistance of $0.008 \mathrm{~K} / \mathrm{W}$ with a pressure difference of only around 0.4 bar.

\subsubsection{Jiang et al: Air and water cooling with sintered bronze particles}

This experiment analysed the effects of sintered porous channels on both air and liquid heat transfer [33]. The channels consisted of sintered bronze particles between $0.5 \mathrm{~mm}$ and $2 \mathrm{~mm}$ in diameter, on a thin copper plate $210 \mathrm{~mm}$ long and $120 \mathrm{~mm}$ wide. The thickness depended on which size particles were used but was between $10 \mathrm{~mm}$ and $12 \mathrm{~mm}$. The system was heated using a serpentine nickel-chromium strip and cooled with air and water separately. The experiment monitored pressure, temperature, and electrical power. The flow rate was recorded, but set to be constant, not requiring monitoring.

The results showed that smaller particle size increased inlet pressure as expected, with the highest pressure for the smallest particle size reaching around $225 \mathrm{kPa}$, compared to the $\sim 150 \mathrm{kPa}$ of the largest particle size for water cooling. Air cooling had a lower overall pressure difference, but the difference between the highest and lowest differences was significantly greater. All of the sintered bronze channels showed significant increase in convective heat transfer coefficient compared to an empty channel, but little effect with relation to each other. It was also found that sintered porous media has better heat transfer than a similar porous media that has not been sintered. 


\subsubsection{Zhang et al: Ball grid array die cooling with nickel-plated copper foam}

This experiment analysed the effect of porosity and pore density in copper foam on heat transfer [34]. Four different porosities: $0.9,0.8,0.7$, and 0.6 , and two different pore densities: 60 pores per inch (PPI) and $100 \mathrm{PPI}$ were used, and in each combination a $13 \mathrm{~mm}$ by $12.2 \mathrm{~mm}$ by $2 \mathrm{~mm}$ block was cut for use as a heat sink. These blocks were nickel plated to prevent corrosion. Deionized water was used as the coolant, and the heat was supplied by four ball grid array dies. The experiment measured flow rate, pressure drop and die temperatures.

The results found that lower porosities lead to higher heat transfer and a higher-pressure differential, while a lower pore density has a similar effect, but with a much more significant increase in pressure with respect to heat transfer. The best performance came from the $60 \mathrm{PPI} 0.7$ sample, which had a thermal resistance as low as $0.3 \mathrm{~K} / \mathrm{W}$, at a pressure of $\sim 35 \mathrm{kPa}$.

\subsubsection{Hetsroni et al: Liquid cooling with sintered stainless steel}

This experiment analysed the effect of porosity on heat transfer in sintered stainless steel [35]. Two different particle sizes were used: $20 \mu \mathrm{m}$ and $60 \mu \mathrm{m}$. The porosities of these samples were measured to be 32 and 44, respectively. These inserts were cut to $5 \mathrm{~mm}$ by $2 \mathrm{~mm}$ by $1 \mathrm{~mm}$. The system was heated by a heater block and cooled using filtered water. The inlet and outlet fluid temperatures, pressure drop across the insert, and flow rate were all measured.

The results show that lower particle size results in a higher pressure over the insert, with a highest pressure of $400 \mathrm{kPa}$ for the $20 \mu \mathrm{m}$ insert vs $250 \mathrm{kPa}$ for the $60 \mu \mathrm{m}$ insert. However, the heat flux also increased with the particle size, with a maximum heat flux of around $6000 \mathrm{~kW} / \mathrm{m}^{2}$ for the $20 \mu \mathrm{m}$ insert, compared with the $\sim 3500 \mathrm{~kW} / \mathrm{m}^{2}$ of the $60 \mu \mathrm{m}$ insert.

\subsubsection{Singh et al: Liquid cooling with sintered copper particles}

This experiment investigated the use of sintered copper granules in heat sinks [36]. The copper granules were 100-200 mesh size and sintered into an inset $14 \mathrm{~mm}$ long by $20 \mathrm{~mm}$ wide and $2 \mathrm{~mm}$ tall. The insert was housed in a glass-topped chamber placed on top of a heating element, and water was passed through it length-ways to cool it. Pressure drop, flow rate, heating input and temperature were all measured. 
The results showed an increase in pressure drop over the chamber with respect to input flow rate. However, this pressure increase was matched by a decrease in thermal resistance. The minimum thermal resistance reached was $0.55 \mathrm{~K} / \mathrm{W}$, at a flow rate of $4.2 \mathrm{~cm}^{3} / \mathrm{s}$ and a pressure drop of $35 \mathrm{kPa}$

\subsubsection{Wan et al: Novel porous heat sink}

This experiment analysed the performance of a novel porous heat sink in heat transfer [37]. The porous media consisted of 200 mesh stainless steel wire, cut into a $20 \mathrm{~mm}$ by $10 \mathrm{~mm}$ by $0.5 \mathrm{~mm}$ block. This block was placed on top of a copper block that had cartridge heaters embedded in it as a heat source, and water was cycled through the block to act as a coolant. Flow rate, heating load, pressure differential, and temperatures were all measured as part of the experiment.

The results showed that increasing the input flow rate had a corresponding increase in the pressure drop, and a corresponding decrease in the thermal resistance. The best results were achieved with a flow rate of $6.2 \mathrm{~cm}^{3} / \mathrm{s}$, where thermal resistances as low as $0.16 \mathrm{~K} / \mathrm{W}$ were achieved with a pressure differential of $35 \mathrm{kPa}$. 


\subsubsection{Comparison table}

\begin{tabular}{|c|c|c|c|c|c|c|c|c|}
\hline 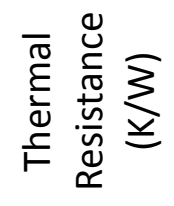 & $\begin{array}{l}\stackrel{n}{N} \\
\dot{m}\end{array}$ & $\begin{array}{l}\varphi \\
\dot{m}\end{array}$ & $\begin{array}{l}\infty \\
\text { ᄋ } \\
\text { ○े }\end{array}$ & 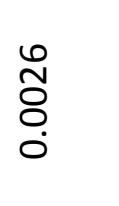 & $\stackrel{m}{0}$ & ने & 占 & $\begin{array}{l}\stackrel{\theta}{-1} \\
\stackrel{0}{0}\end{array}$ \\
\hline 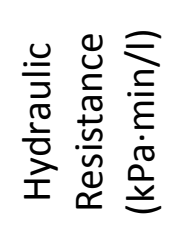 & 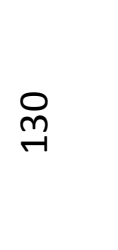 & $\underset{\sim}{\stackrel{\sigma}{\rightarrow}}$ & $\stackrel{\dot{\sim}}{\sim}$ & $\begin{array}{l}\frac{0}{0} \\
\frac{\pi}{0} \\
\frac{\pi}{\pi} \\
\frac{1}{2} \\
0 \\
\frac{0}{2}\end{array}$ & ָั่ & $\underset{\leftarrow}{\stackrel{్}{G}}$ & $\stackrel{\text { m}}{\rightarrow}$ & $\begin{array}{l}\infty \\
\stackrel{-}{\sigma}\end{array}$ \\
\hline 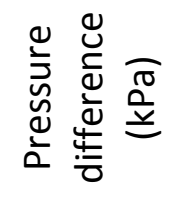 & $\stackrel{\sim}{\sim}$ & $\begin{array}{l}\mathscr{\varphi} \\
\mathscr{q}\end{array}$ & 우 & 윰 & $\stackrel{\varphi}{m}$ & ঠ̊ণ & $\stackrel{\varphi}{m}$ & $\stackrel{\mathscr{N}}{\sim}$ \\
\hline 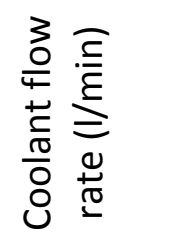 & $\begin{array}{l}\text { ○े } \\
\text { ○े }\end{array}$ & $\begin{array}{l}\text { Oִ } \\
\text { r }\end{array}$ & $\stackrel{r}{r}$ & $\begin{array}{l}\frac{0}{0} \\
\frac{0}{0} \\
\frac{0}{\pi} \\
\frac{\pi}{2} \\
0 \\
0\end{array}$ & $\underset{\sim}{\sim}$ & 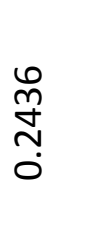 & $\begin{array}{l}\text { Nָ } \\
\text { ஸ் }\end{array}$ & $\begin{array}{c}\stackrel{N}{m} \\
0\end{array}$ \\
\hline 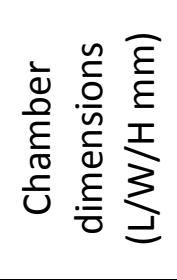 & $\begin{array}{l}\stackrel{\circ}{\ominus} \\
\text { ○े } \\
\text { ㅇํ }\end{array}$ & 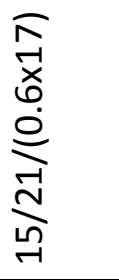 & 옹 & 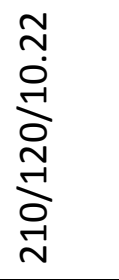 & 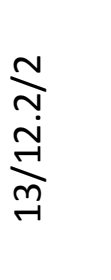 & $\frac{\vec{\gamma}}{\text { ம) }}$ & 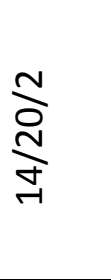 & $\begin{array}{l}\text { ñ } \\
\text { Oे } \\
\text { ô } \\
\text { ồ }\end{array}$ \\
\hline 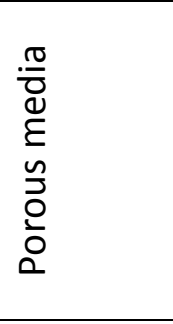 & 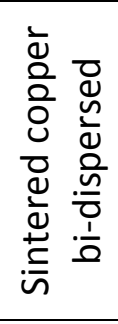 & 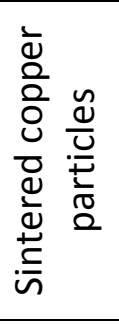 & 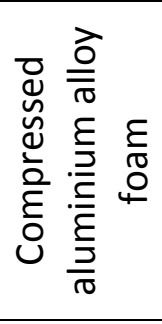 & 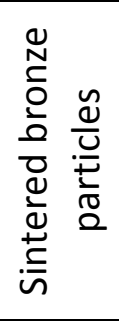 & $\begin{array}{l}\frac{\varepsilon}{\pi} \\
0 \\
\frac{0}{4} \\
\frac{0}{0} \\
\frac{0}{2} \\
0\end{array}$ & 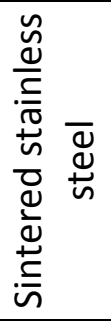 & 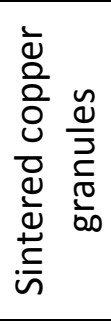 & 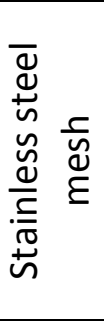 \\
\hline 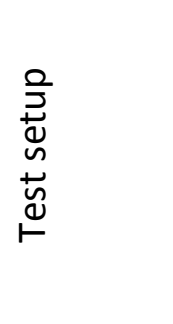 & 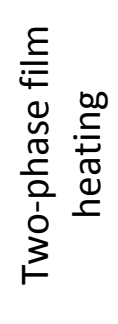 & 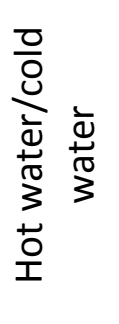 & 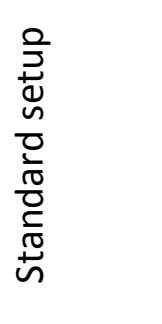 & 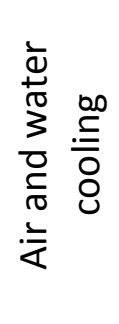 & 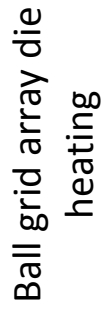 & 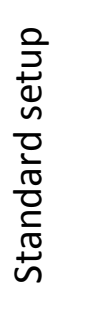 & 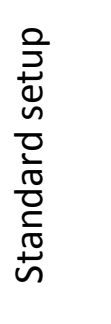 & 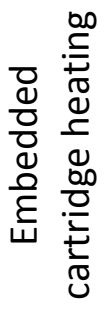 \\
\hline$\frac{\bar{O}}{\frac{D}{ \pm}}$ & 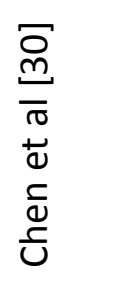 & 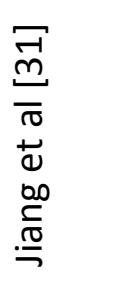 & 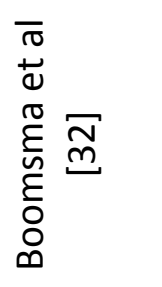 & 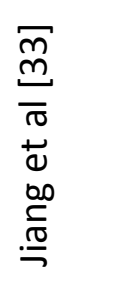 & 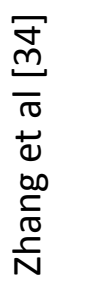 & 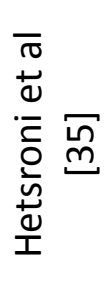 & 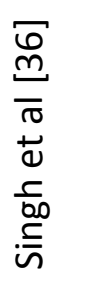 & 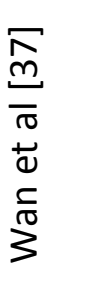 \\
\hline
\end{tabular}

Table 2: Table of previous research. [38] 


\subsection{Previous work with porous silver at Victoria University}

While research into porous media has covered a variety of materials and fabrication methods, this project has a particular focus on freeze cast silver (FCS) surfaces. These are a novel form of porous media, and research on the subject has taken place almost exclusively at Victoria University. Prior to this thesis, research involving these surfaces has been focussed on twophase cooling applications. The fabrication process required to produce FCS surfaces will be explained in chapter 4 , but their heat transfer performance will be discussed here.

FCS surfaces have shown to have a strong positive effect on pool boiling. Research by Gouws and Shortt found that FCS silver surfaces increased the heat flux through a copper disk by as much as 5.3 times [28]. They also lowered the temperature at which heat flux began to increase by up to 3.7 degrees. The best improvements were found to be when the concentration of silver was highest.

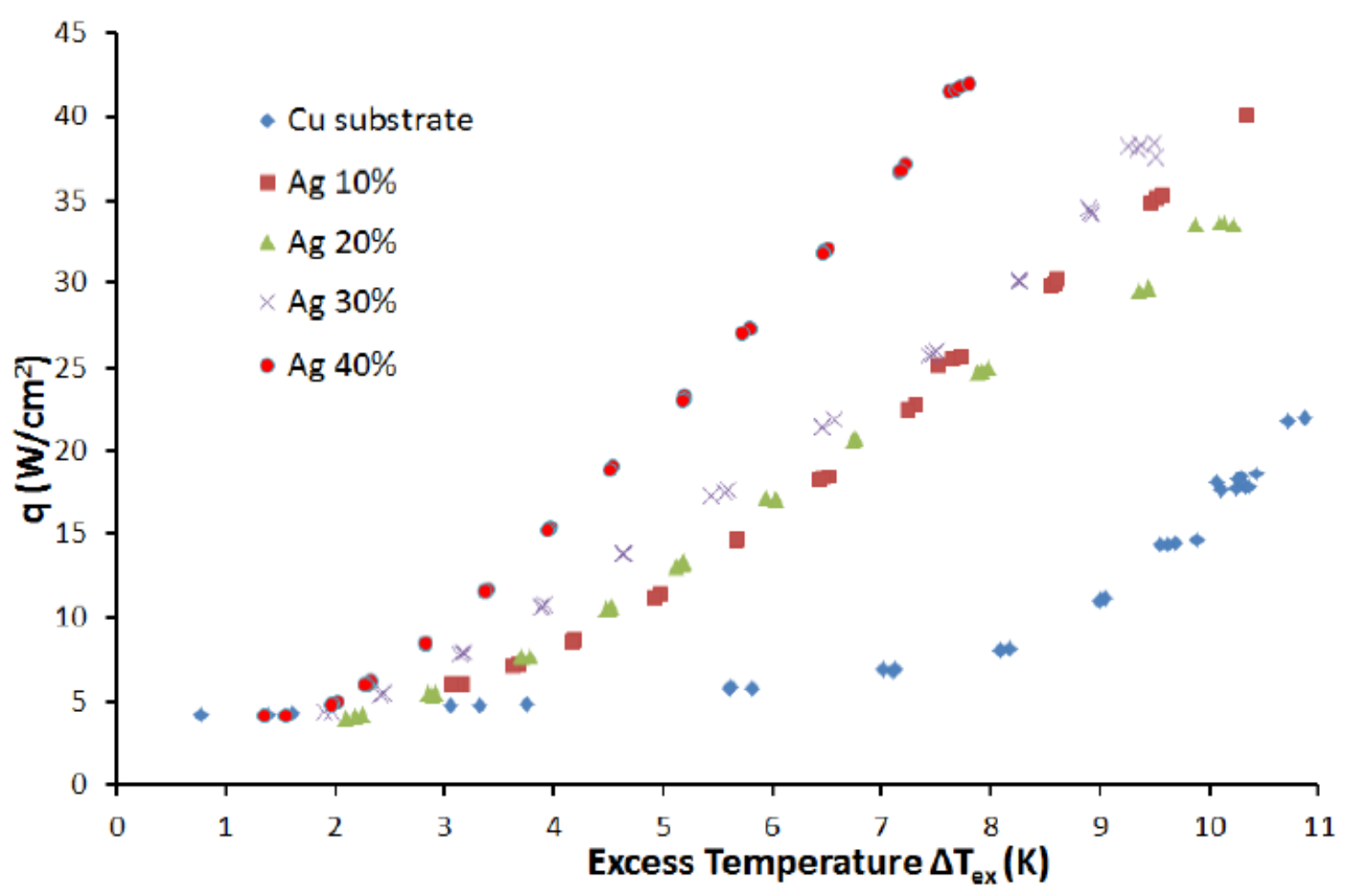

Figure 10: Heat flux vs excess temperature for various silver concentrations, as well as bare copper. [28]

This research also categorized the microstructures of the FCS into three distinct pore sizes. Type A pores consisted of large $(100 \mu \mathrm{m}-200 \mu \mathrm{m})$ pores with an irregular shape and random 
alignment and have since been attributed to the presence of air bubbles in the solution during freezing. Type B pores consisted of $40 \mu \mathrm{m}-80 \mu \mathrm{m}$ long channels, approximately $10 \mu \mathrm{m}$ wide and deep, that align with neighbouring Type B pores. These are likely the result of ice crystals carving paths through the metal particles. Finally, Type $\mathrm{C}$ pores are the pores between sintered particles, and are between $0.5 \mu \mathrm{m}$ and $5 \mu \mathrm{m}$ in width.
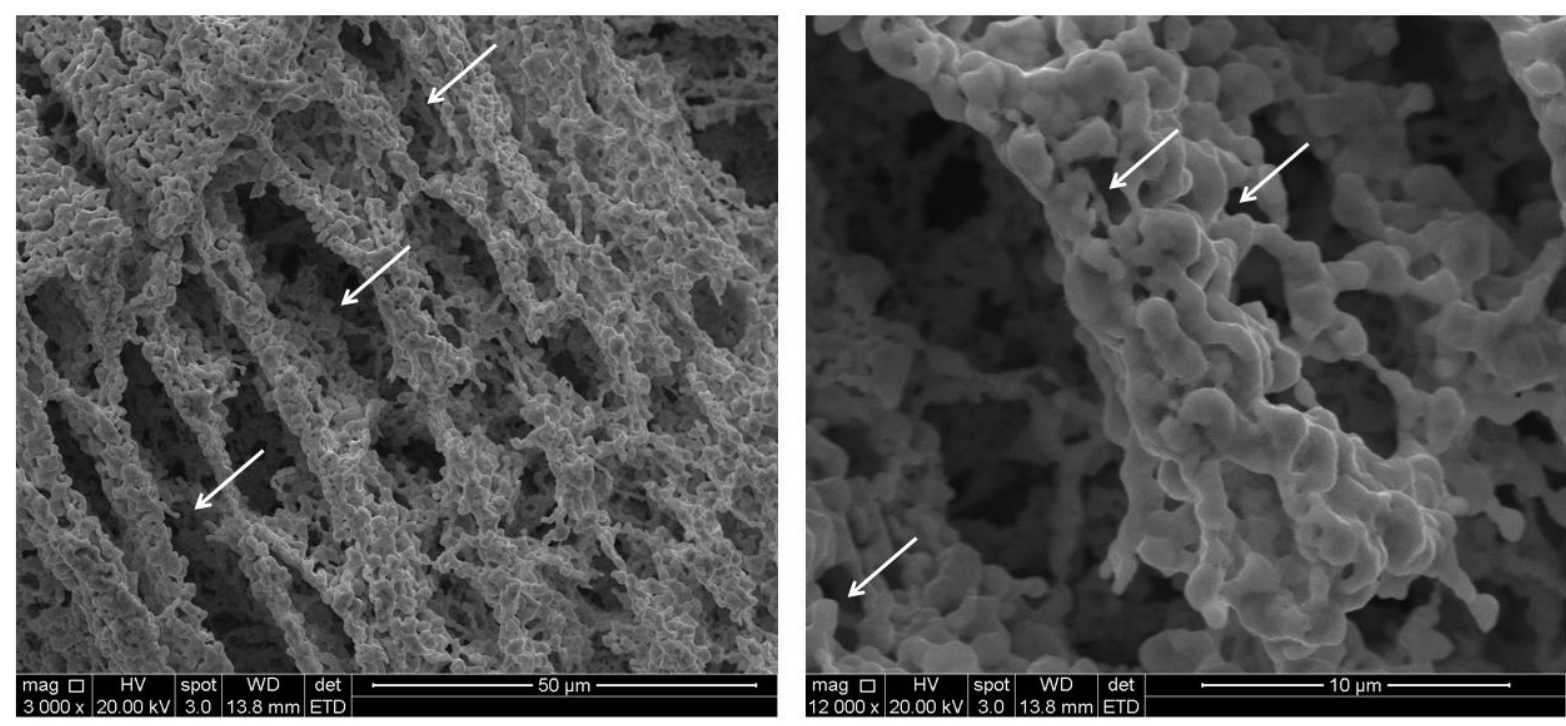

Figure 11: Electron microscope pictures of type B (left) and type C (right) pores.

During a following experiment, a silver surface was produced using a $40 \%$ silver solution but was not freeze cast before sintering. This surface performed better than plain copper but considerably worse than the freeze cast surface with the same silver concentration. 


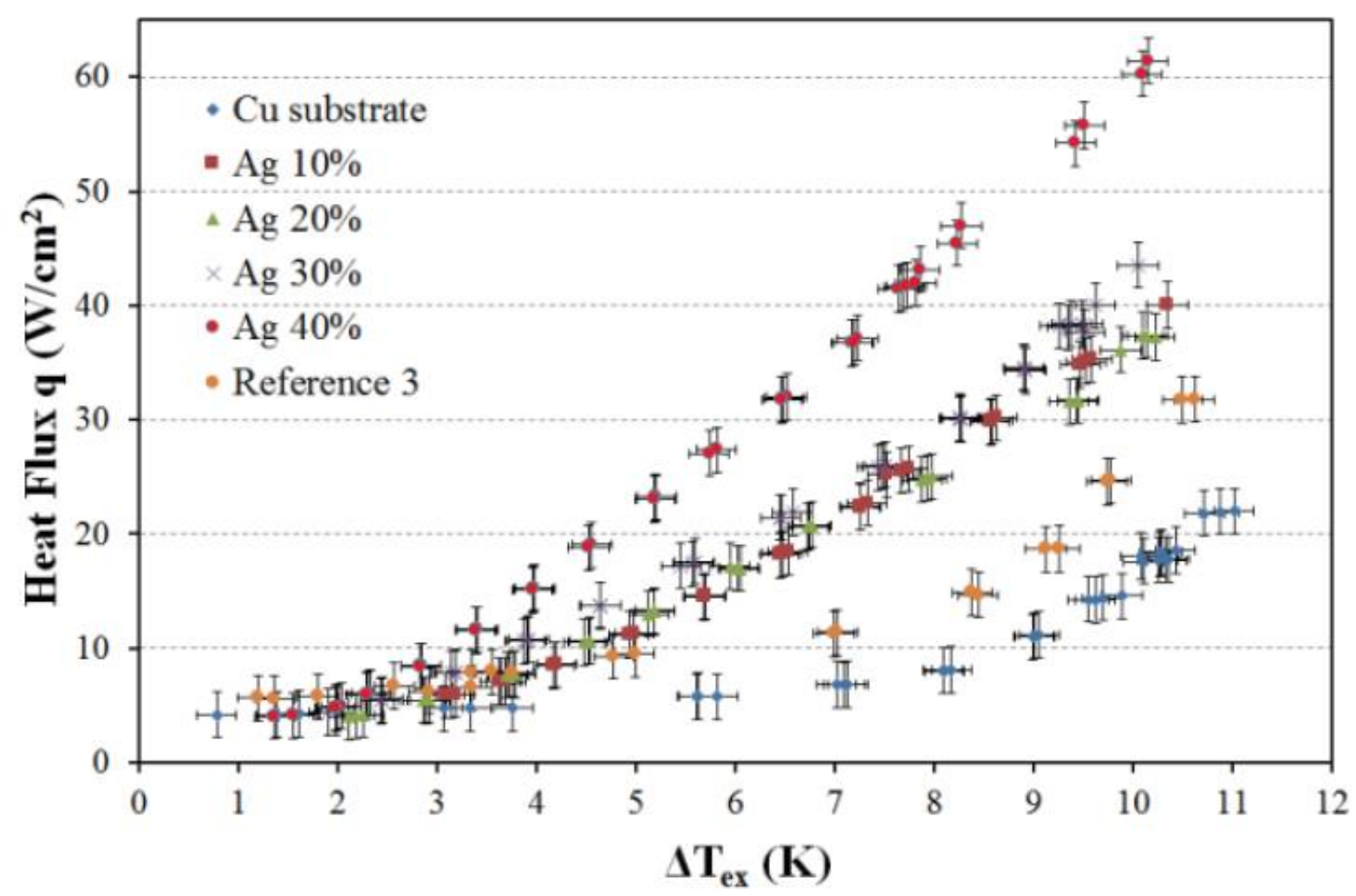

Figure 12: Heat flux vs excess temperature for plain copper, FCS surfaces with various silver concentrations, and a sintered silver surface with $40 \%$ silver concentration (Reference 3). [39]

These tests also discovered that during the first pool boiling test with fresh surfaces, the heat flux was always lower than in subsequent tests. As a result of this, future surfaces were boiled in DI water prior to being used. 


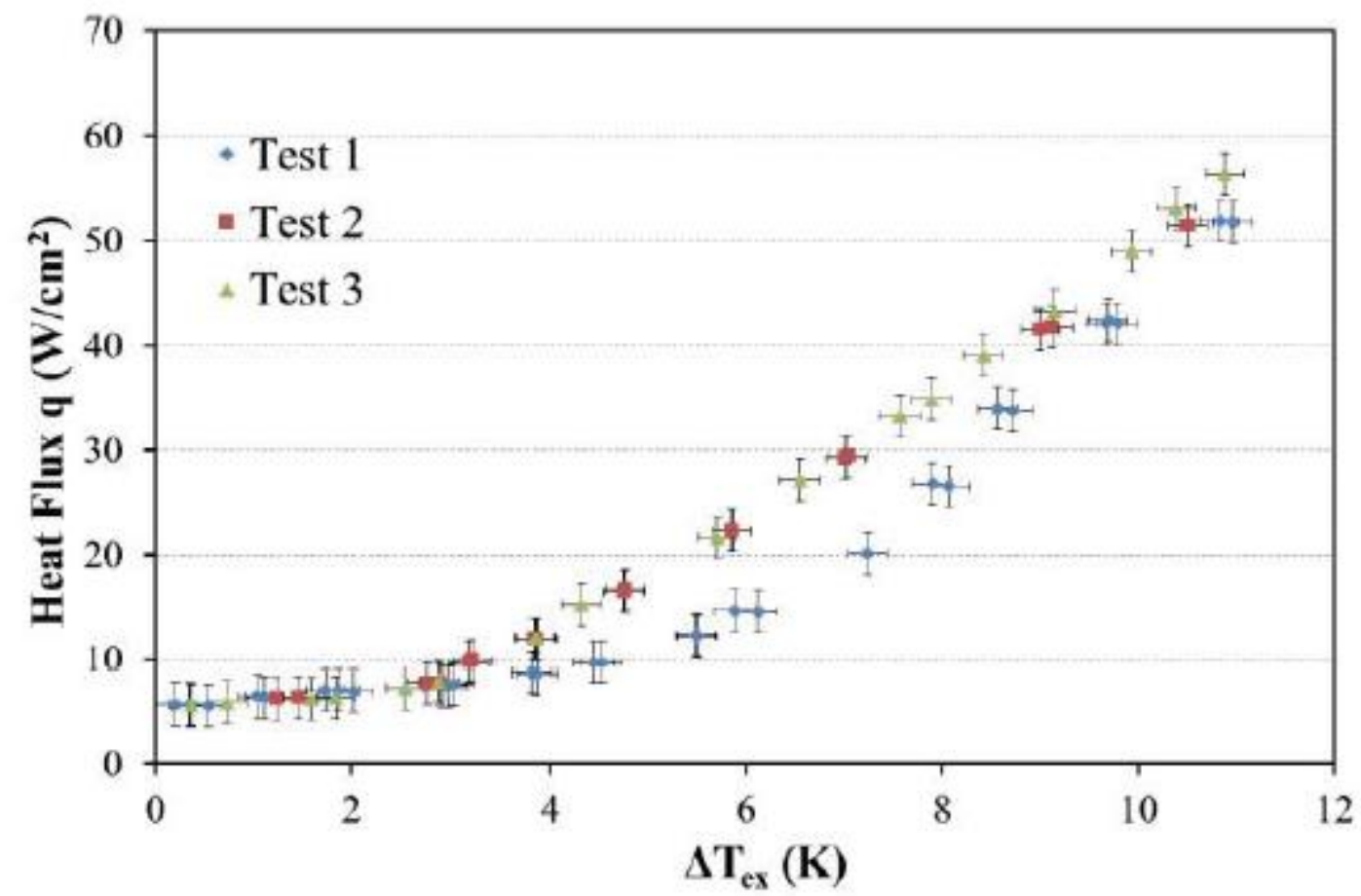

Figure 13: Heat flux vs excess temperature for three consecutive tests. Note the consistently lower heat flux for test 1. [39]

During this research it became apparent that the freeze cast surfaces had variable wettabilities. Because wettability can affect heat transfer, additional research was carried out to determine factors in fabrication that influence wettability. Surfaces were produced with a range of silver concentrations and their contact angles were measured. It was discovered that a higher concentration of silver in the initial solution produced a surface with a lower contact angle [39]. However, it was also noted that the silver surfaces change contact angle over long periods of time. It is suspected that this is due to a change in surface chemistry occurring. 


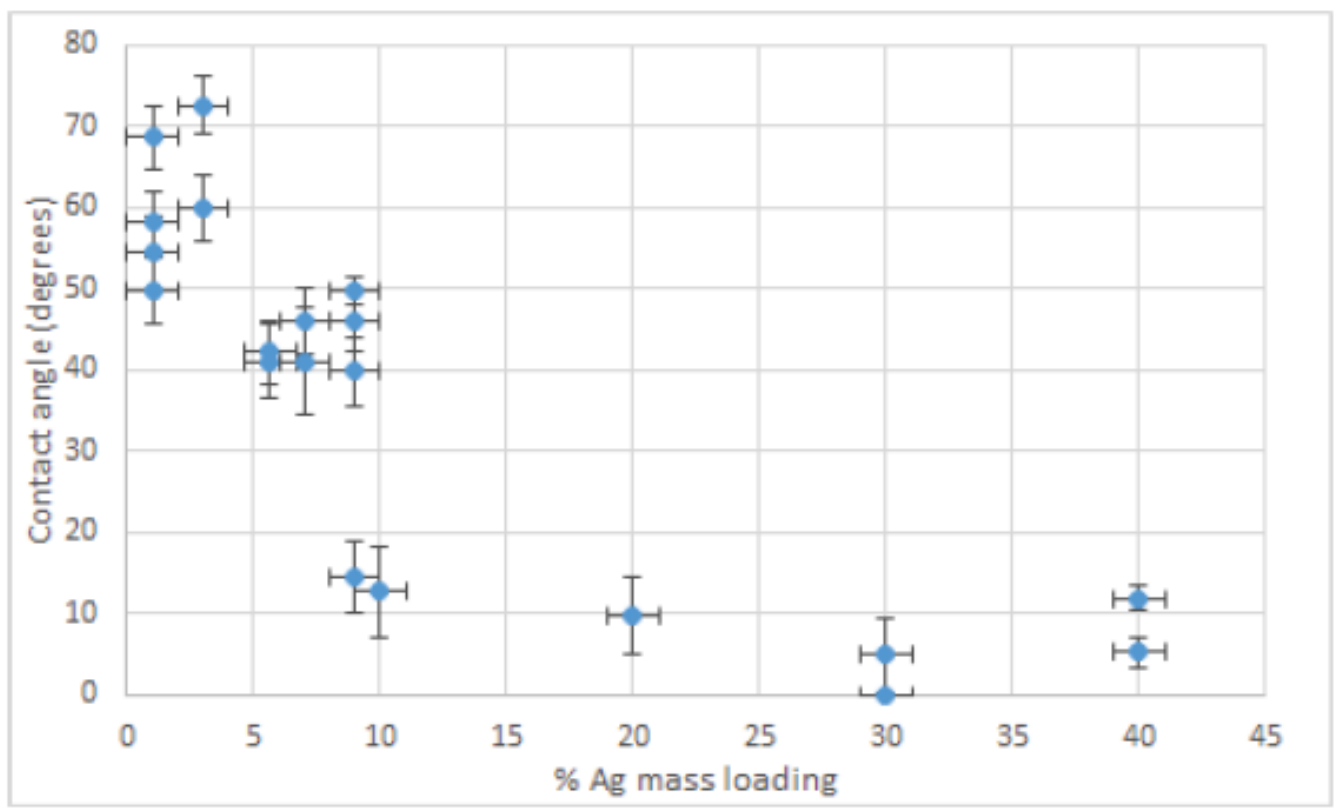

Figure 14: Contact angle vs silver concentration in FCS surfaces. [39]

Finally, experiments were carried out measuring the lifetime of a sessile droplet as it was evaporated off a surface at temperatures between $75^{\circ} \mathrm{C}$ and $120^{\circ} \mathrm{C}$, and this was compared to the contact angle of the surface. This research discovered that hydrophobic surfaces require much longer to evaporate a droplet than hydrophilic ones [39].

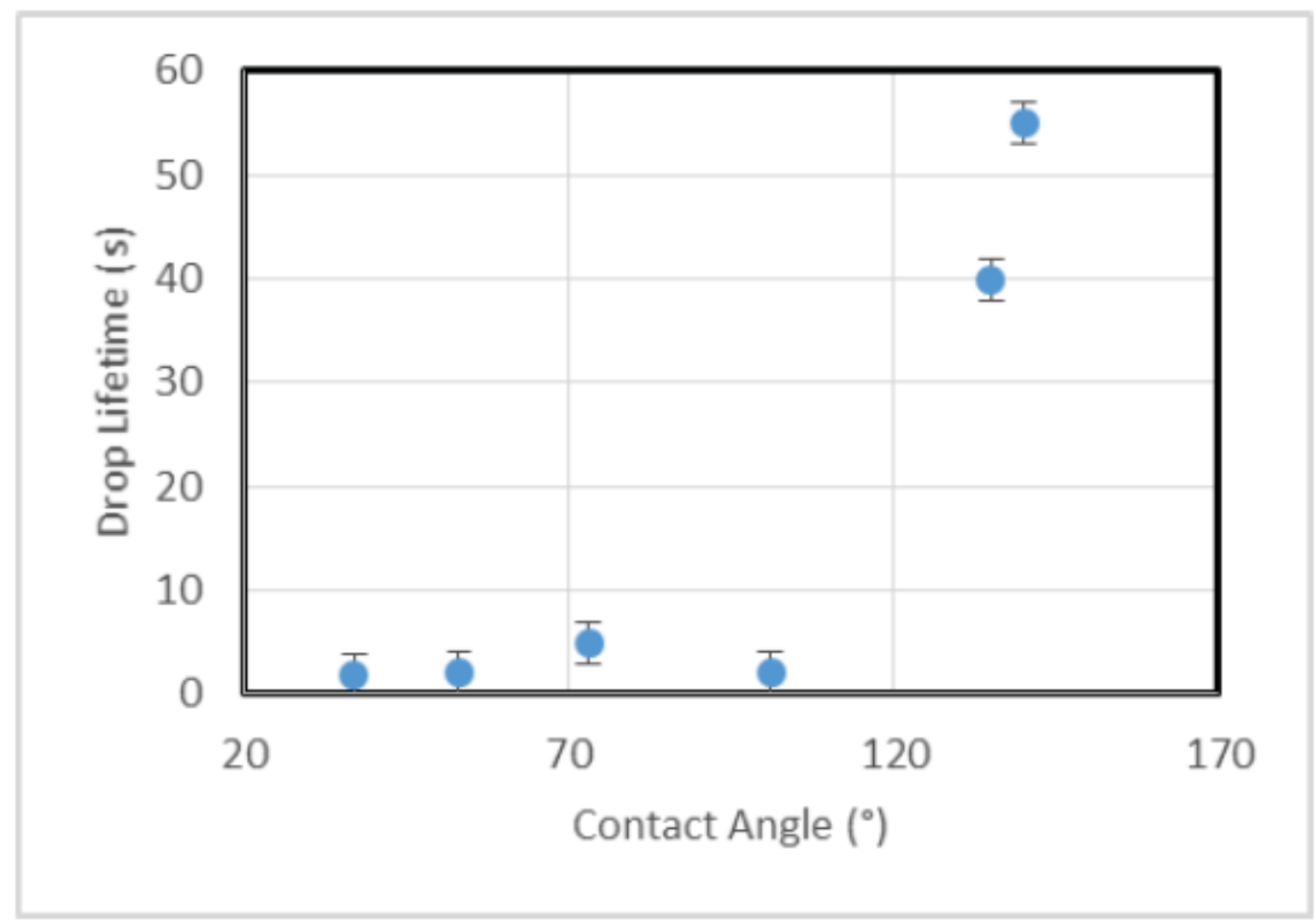

Figure 15: Drop lifetime vs contact angle of FCS surfaces. [39] 
As previously mentioned, this research focussed mainly on the performance of FCS surfaces in two-phase applications. However, the results clearly indicate that their heat transfer properties are superior to plain copper in two-phase systems, and it is expected that these properties are similarly advantageous in liquid cooling applications.

\subsection{Summary}

Thermal resistance is the method by which cool system performance is characterised. The goal of designing a cooling system is to minimise its thermal resistance. Thermal resistance is inversely proportional to both the surface area being cooled and convective heat transfer coefficient. The convective heat transfer coefficient is most easily increased by increasing the thermal conductivity of the fluid involved in convection, or by decreasing the characteristic length of the surface over which the convection is occurring.

Microchannels are extremely useful for reducing the thermal resistance of cooling systems, as they both increase the surface area being cooled and decrease the characteristic length of the said surface. However, reducing the characteristic length too much will increase the hydraulic resistance, which will impede flow. If the hydraulic resistance is too high, the reduction of characteristic length will be cancelled out by the reduction of fluid velocity, and the thermal resistance will increase accordingly.

Previous research into freeze cast silver porous microstructures has shown positive results in improving heat transfer in phase-change based experiments. Their high surface area, low characteristic length, and proven effectiveness in boiling tests indicates that they may also improve convective heat transfer in liquid cooling applications. 



\section{Test system for forced convective heat transfer}

This chapter provides details of the test system designed and constructed during the project. The basic components of an active closed-loop liquid cooling system are discussed in section 3.1. Details of the sensors, including their purpose, calibration, and implementation, are included in section 3.2. The integration of these components into a single system, with respect to both additional hardware and software is outlined in section 3.3. Finally, the procedure for performing a test is detailed in section 3.4.

\subsection{Cooling system}

As established in section 2.3, active closed-loop liquid cooling systems require a pump to drive fluid flow and a heat exchanger to cool the liquid for reuse, and some include a reservoir to ensure that sufficient coolant is always available. These elements are all present in the system used in this project, which also includes a heat source, a specially designed cooling chamber which contains the cold plate, and various sensing elements to gather data.

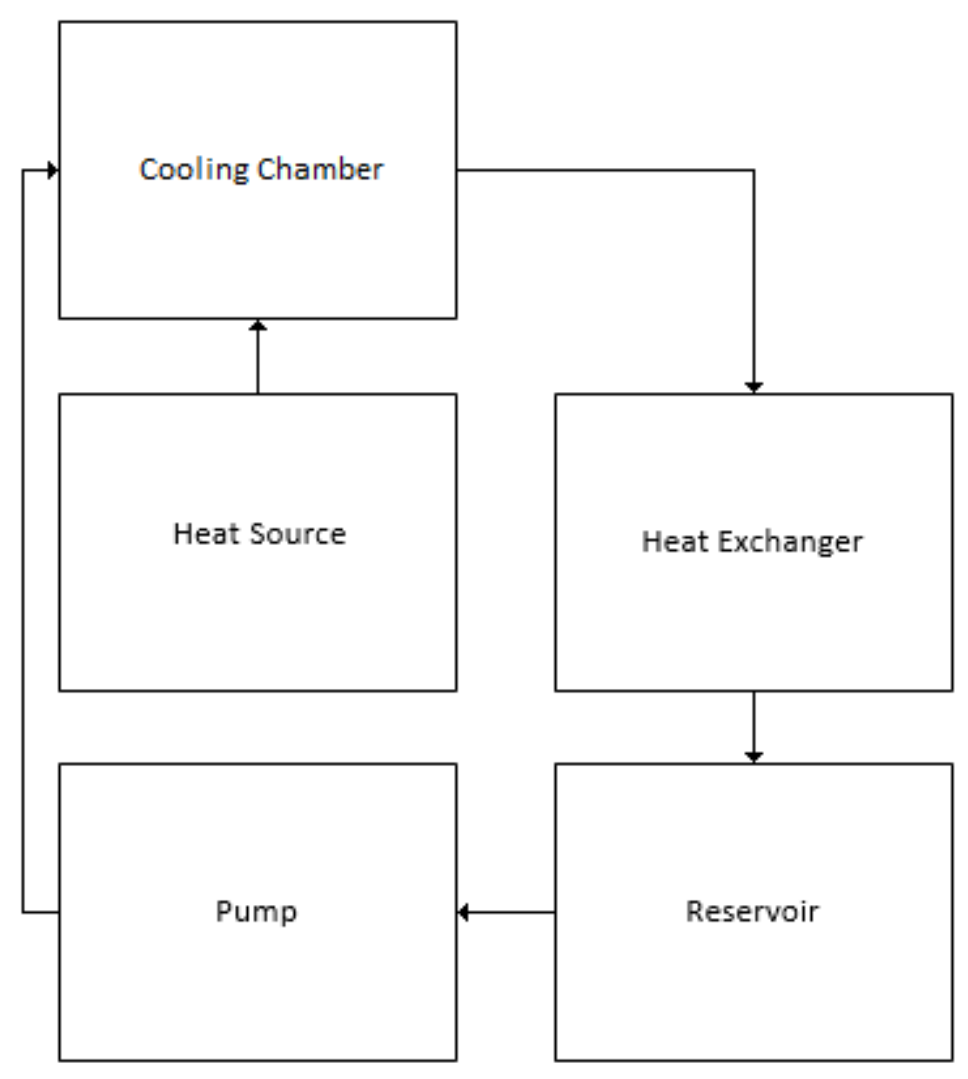

Figure 16: Block diagram of the cooling system. 


\subsubsection{Heat source and heat flux meter}

The heat source consists of a copper column $30 \mathrm{~mm}$ in diameter and $69.5 \mathrm{~mm}$ tall, with four $250 \mathrm{~W} 55 \mathrm{~mm}$ cartridge heaters embedded in the base. Copper was chosen because of its good thermal conductivity, sitting at $399 \mathrm{~W} / \mathrm{m} \cdot \mathrm{K}$ [40]. This assembly rests on top of a $13 \mathrm{~mm}$ thick square ceramic base, made of Macor machinable ceramic. This ceramic has a thermal conductivity of $1.46 \mathrm{~W} / \mathrm{m} \cdot \mathrm{K}$ [41] and should ensure that most of the power from the heaters cannot flow downwards. Radially surrounding the column is a $34.5 \mathrm{~mm}$ thick layer of Yulang Wool ceramic fibre board insulation to increase thermal resistance in the radial direction. This insulation has a significantly lower thermal conductivity than the copper it surrounds, at around $0.065 \mathrm{~W} / \mathrm{m} \cdot \mathrm{K}[42]$, ensuring that heat flow is mostly one-dimensional. Four holes were drilled into the side of the column to house thermocouples, and a shaft through the insulation was cut to allow these thermocouples to pass through it. These four thermocouples are used to create a heat flux meter, which will be discussed in more detail in section 3.2.3. On the sides of the insulation are four threaded rods which allow for the system to be clamped down once the cooling chamber is in place. 


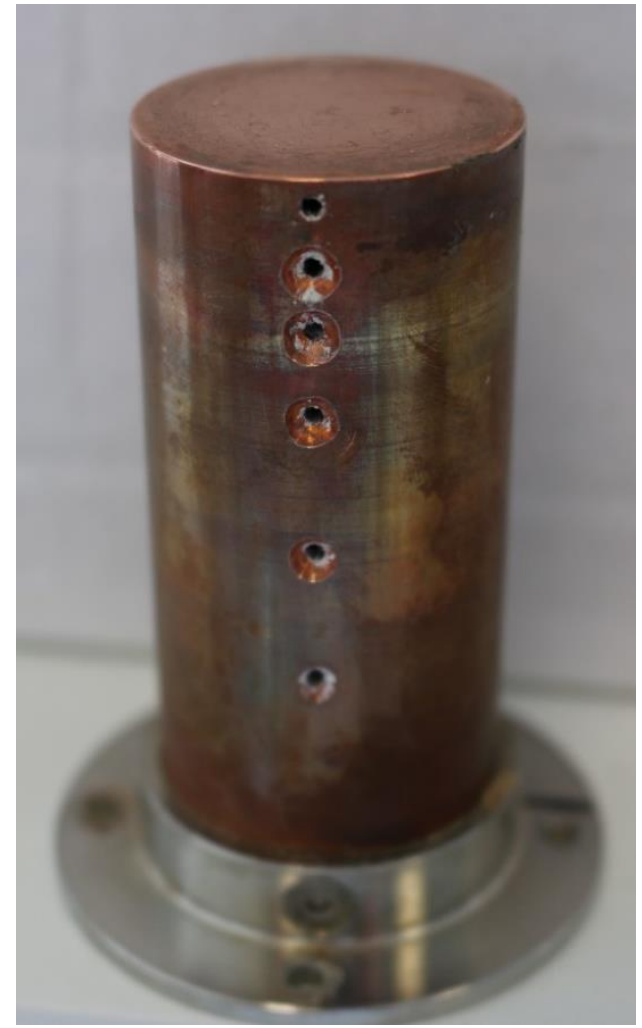

Figure 17: The heater column. The top four holes are used by the heat flux meter. The bottom two holes were unused in this project, but thermocouples could be placed into them to record the temperature between the four heating elements.

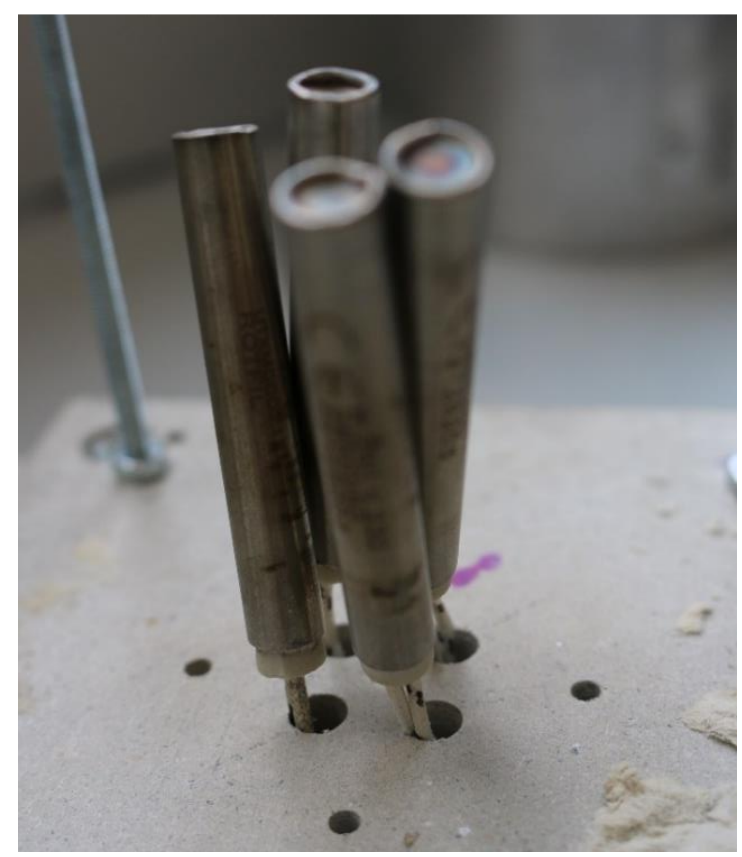

Figure 18: The four cartridge heaters. 


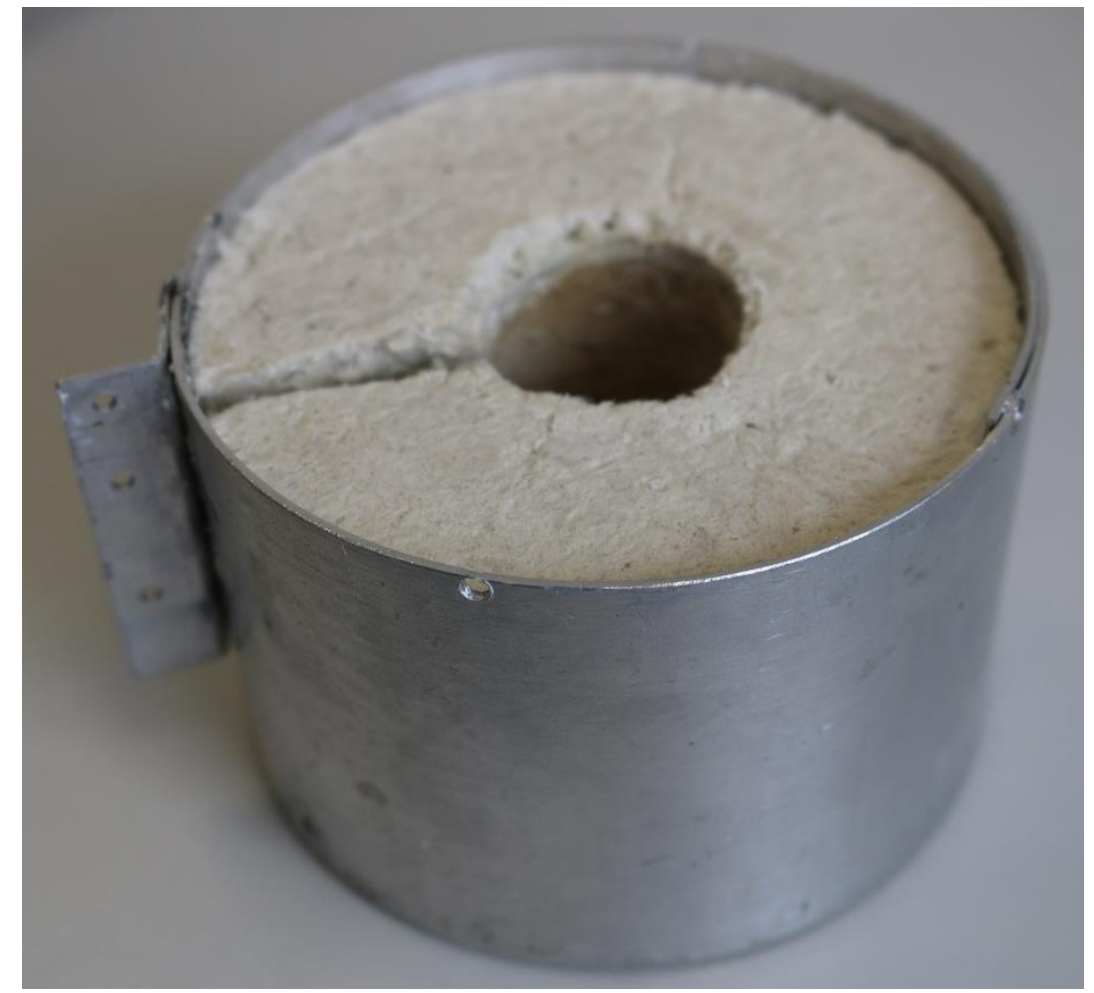

Figure 19: The ring of fibre insulation, with a notch on one side where the thermocouples could be threaded into the heating column.

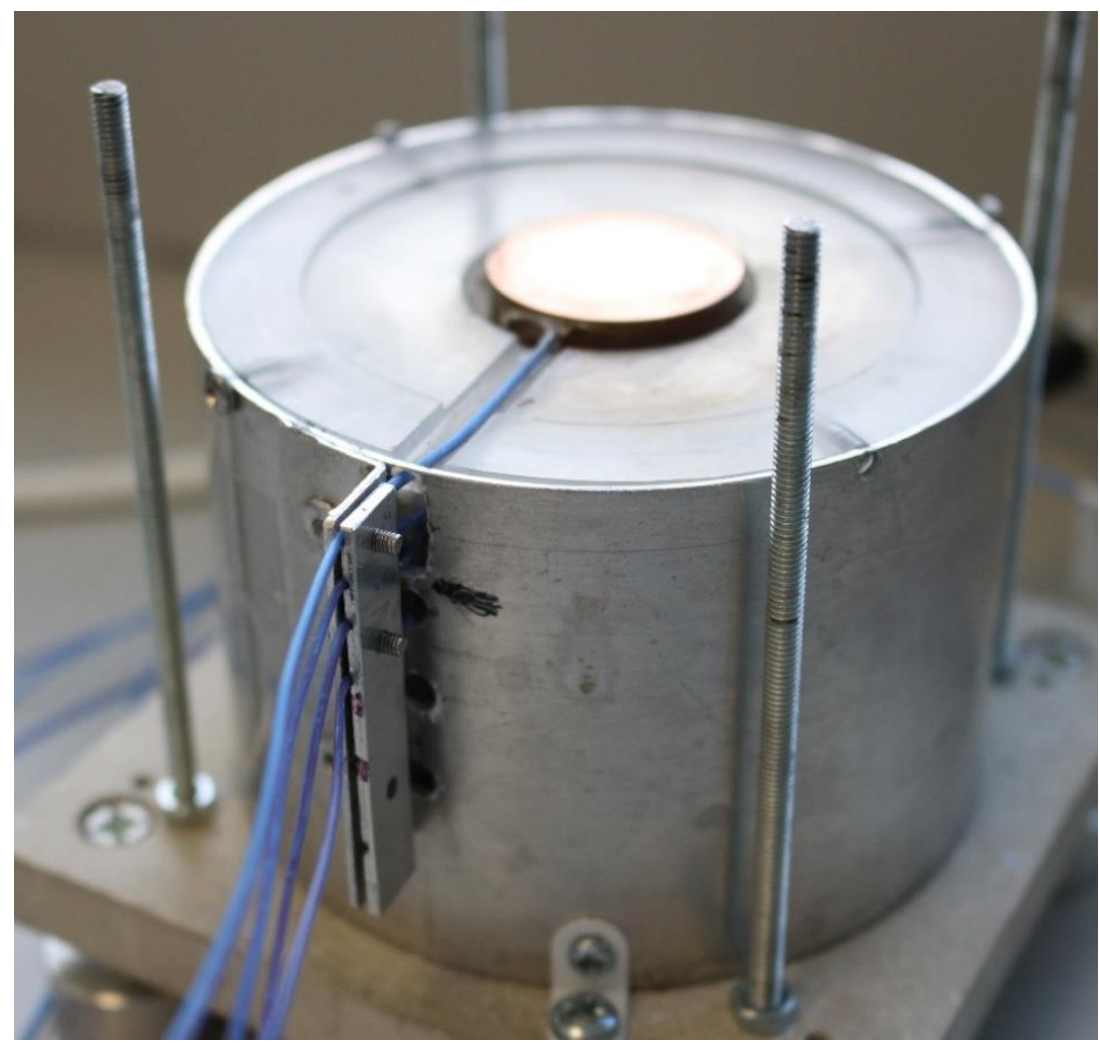

Figure 20: The heat source fully assembled. 


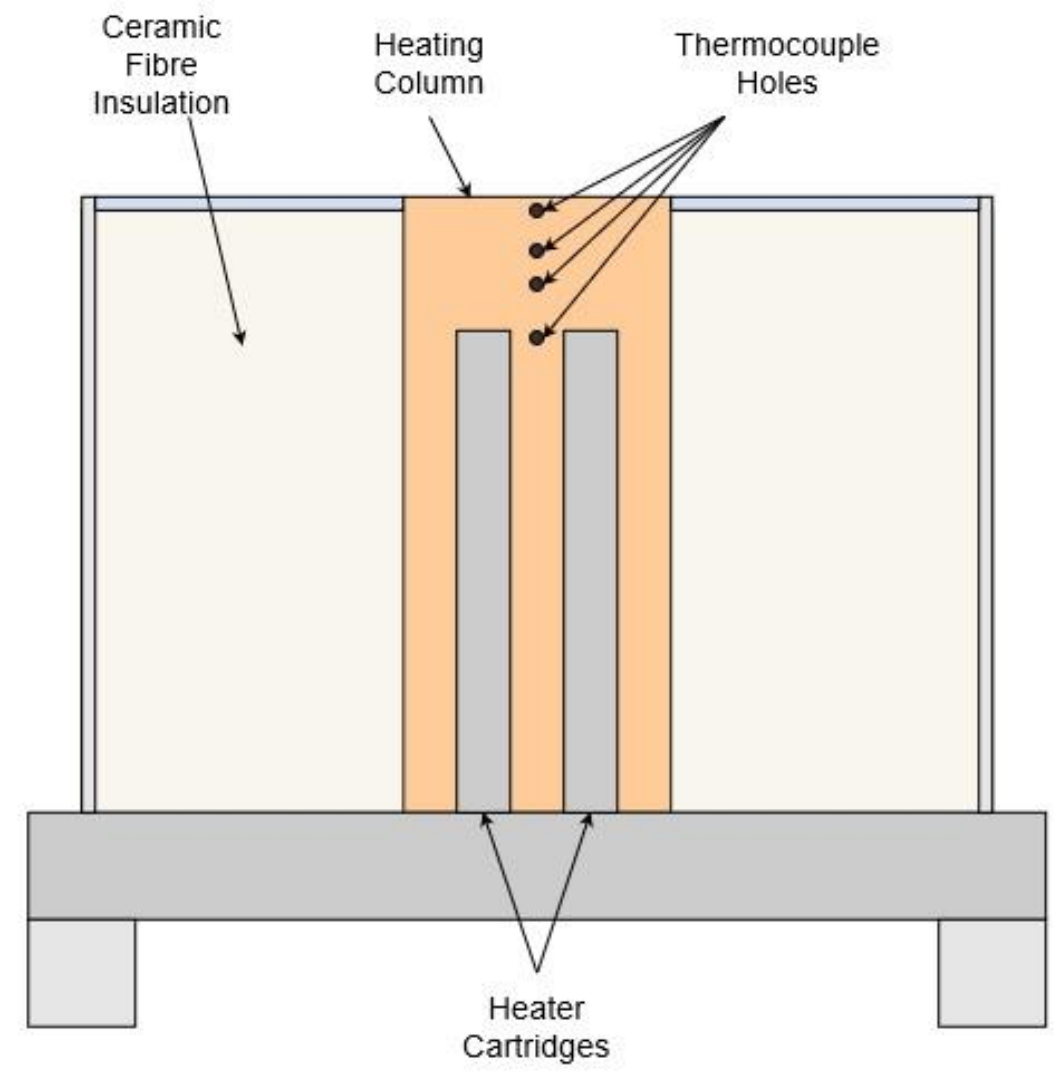

Figure 21: Heat source cross-section.

\subsubsection{Cooling chamber}

The cooling chamber consists of a polyether ether ketone (PEEK) hollow cylinder $11 \mathrm{~mm}$ tall, with an internal diameter of $18 \mathrm{~mm}$. PEEK was used because it is a rigid material with a high melting point $\left(343^{\circ} \mathrm{C}\right)$ and a reasonably low thermal conductivity $(0.25 \mathrm{~W} / \mathrm{m} \cdot \mathrm{K})$. O-rings and threaded holes are positioned on the top and bottom to allow special plugs to be attached, and two M1/16 male tubing connectors are attached at $90^{\circ}$ angles to each other, to act as an inlet and outlet. 


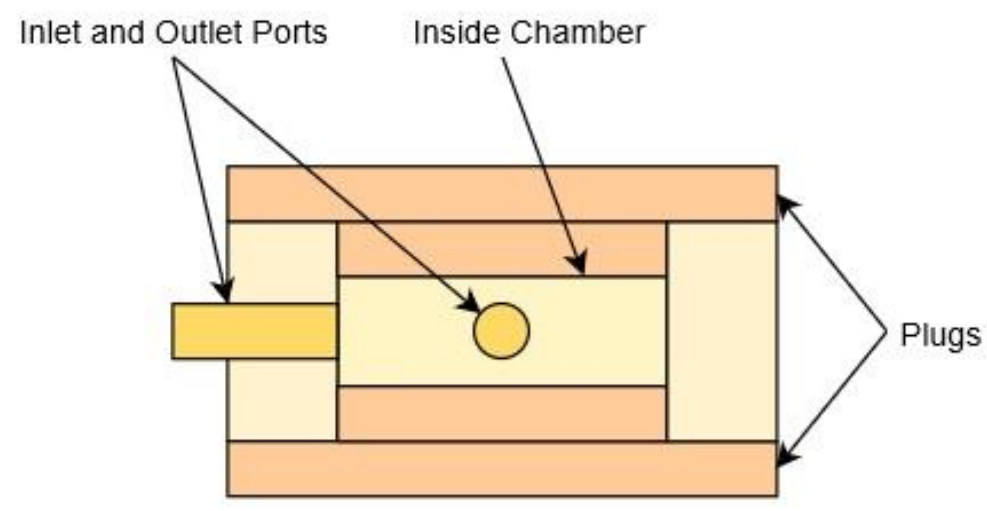

Figure 22: Chamber cross-section.

The plugs are made of copper, again due to its high thermal conductivity, and consist of an $18 \mathrm{~mm}$ in diameter by $3 \mathrm{~mm}$ tall disk on top of a wider $29 \mathrm{~mm}$ in diameter by $2.5 \mathrm{~mm}$ tall disk. The second disk has four countersunk screw holes to allow the screws that attach it to the chamber to lie flat, and a hole in one side for a thermocouple. After being machined, these plugs were degreased with acetone and isopropanol, and etched in acetic acid to remove any surface oxides.

The plugs are designed to be interchangeable, and the porous structures are fabricated directly onto the plugs. The plug on the bottom of the chamber acts as the cold plate and will be referred to as the substrate. This plug is attached to the heating column via a thermal interface material, which will be discussed in section 5.1.1, and the whole chamber is held in place via a brace attached to the threaded rods on the heat source.

Two special plugs were fabricated to act as the roof of the chamber. One was cut from clear plastic to allow for vision into the chamber. The other was made with a third male tubing connector protruding vertically from its centre, to allow for tests to be carried out comparing vertical flow to horizontal flow. This configuration uses both connectors on the chamber itself as outlets. 


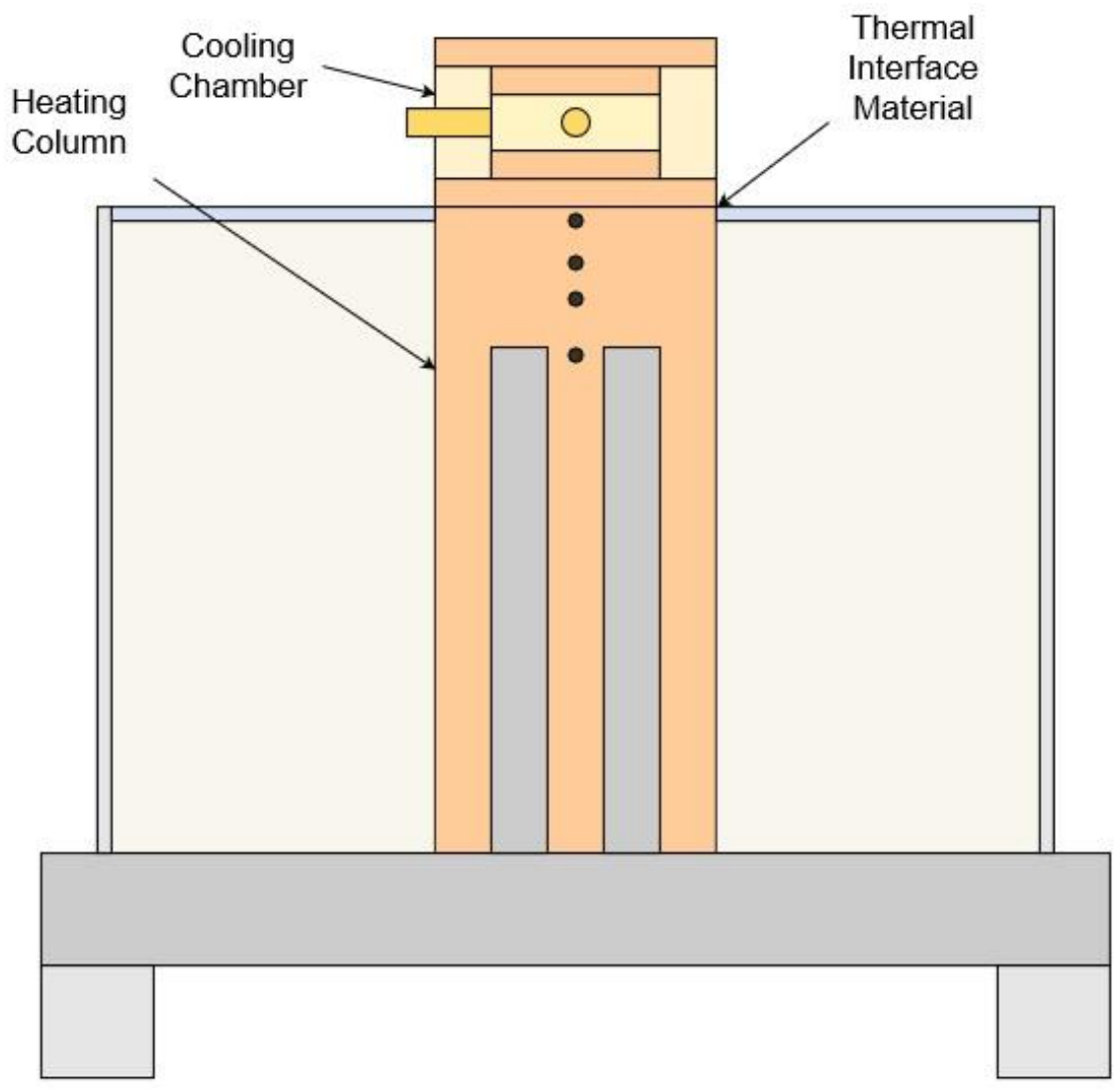

Figure 23: Cross-section of fully assembled system.

\subsubsection{Pump}

The pump used is a Favson F312 diaphragm pump, which is rated to be able to pump up to $4 \mathrm{~L} / \mathrm{min}$ at $12 \mathrm{~V}$ [43]. Diaphragm pumps are a form of positive displacement pump that use the expansion and compression of a diaphragm to pull and push fluid through a pair of valves at the inlet and outlet, respectively. They have the advantage of being able to operate for long periods of time without significant wear, which was necessary for the test system as tests being run tend to take a while to settle between voltage settings. 

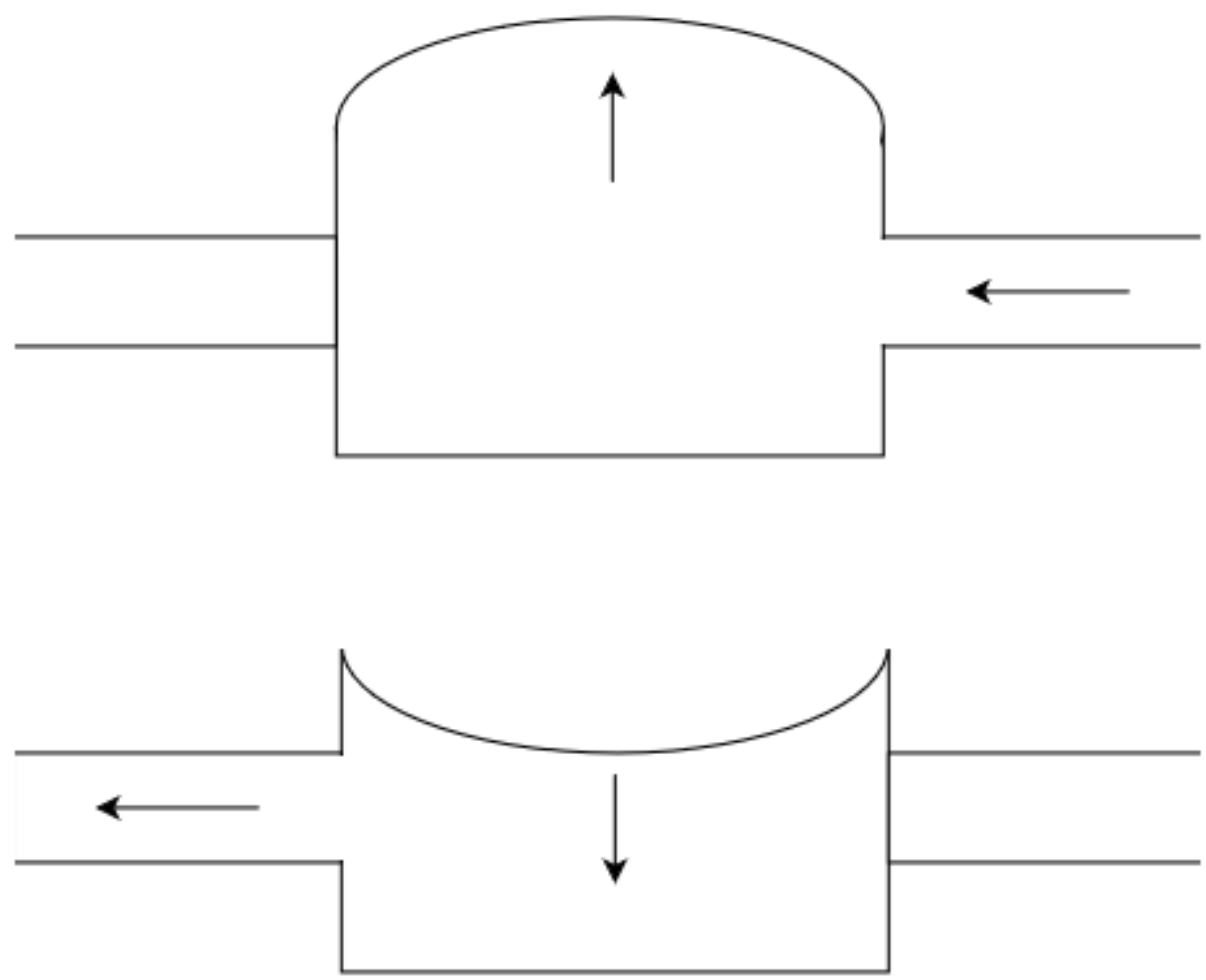

Figure 24: The operation of a diaphragm pump. As the diaphragm expands (top), liquid is pulled into the chamber while the outlet is sealed. Then, as it contracts, the inlet is sealed, and liquid is pushed out via the outlet.

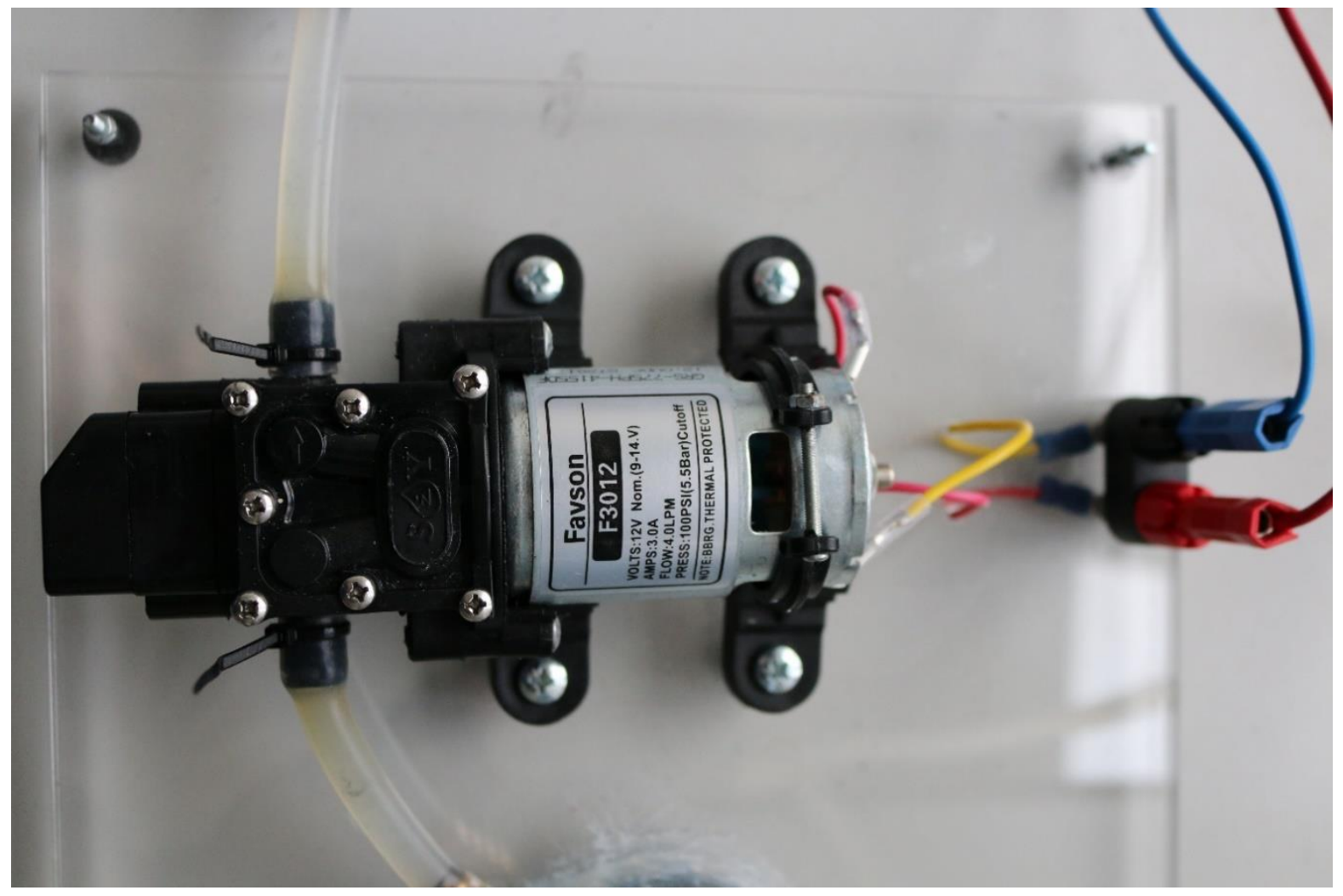

Figure 25: The Favson F312 pump. 
The free flow characteristics of the pump were found by pumping water into a reservoir for a period of one minute, and recording the mass displaced over that period. From this, the flow rate could be calculated by dividing the mass by the density of water $\left(\rho=1000 \mathrm{~kg} / \mathrm{m}^{3}\right)$ to find the volume and then dividing that by the time taken.

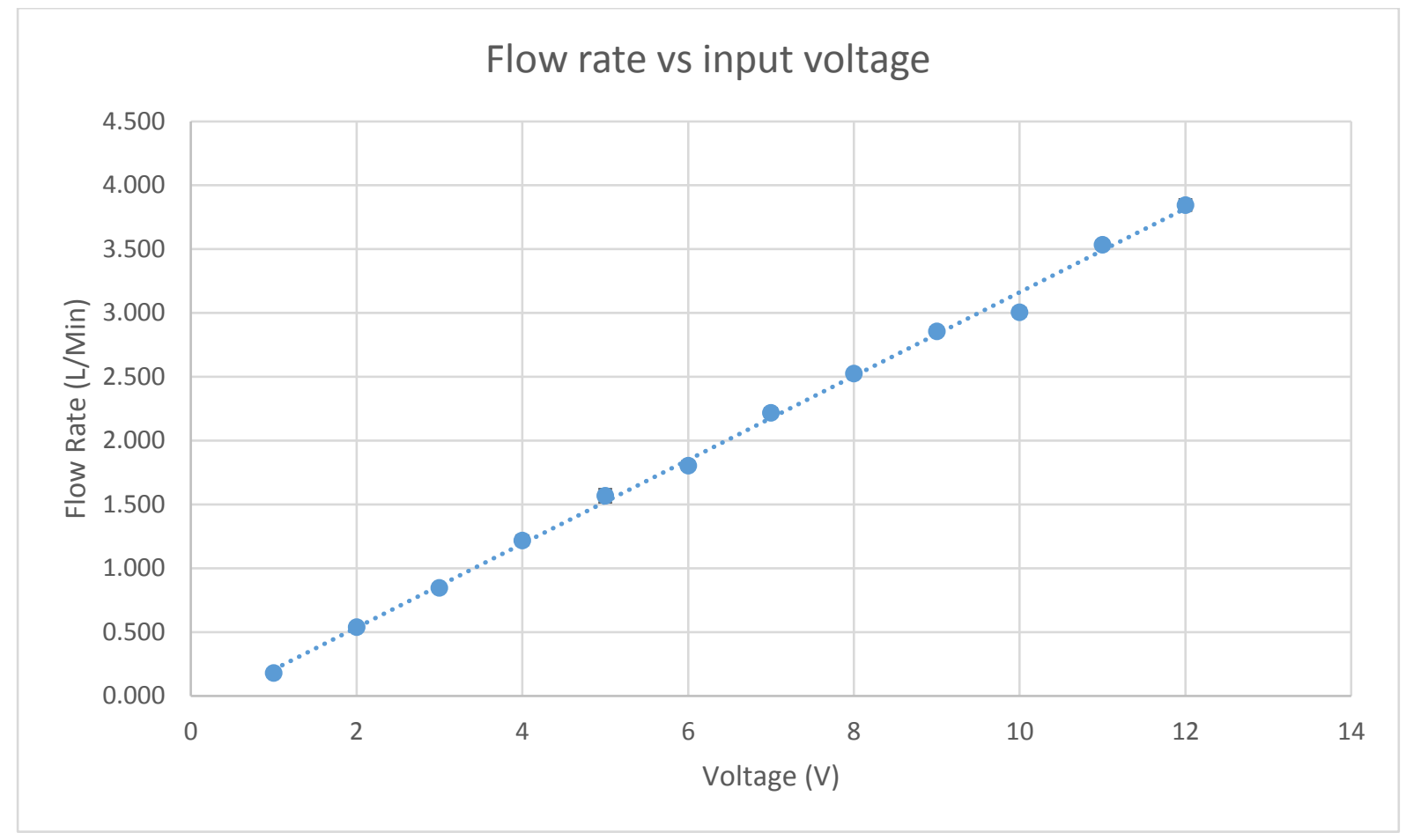

Figure 26: Pump free flow characterization.

\begin{tabular}{|c|c|}
\hline Flow rate $=\left(\begin{array}{c}\text { Scaling } * \text { input voltage })+ \\
\text { offset. }\end{array}\right.$ & Flow rate $(\mathrm{L} / \mathrm{min})$ \\
\hline Scaling & 0.3283 \\
\hline Offset & -0.1211 \\
\hline
\end{tabular}

Table 3: The pump's free-flow characteristics.

The free flow rate as a function of input voltage is approximately linear.

\subsubsection{Heat exchanger}

The heat exchanger consists of a $0.67 \mathrm{~m}$ long copper pipe, coiled into a helix and submerged in a Polyscience temperature-controlled circulating bath set to $20^{\circ} \mathrm{C}$. Compared to the heat exchangers used in typical closed-loop cooling systems this is overkill, but this decision was purposeful. By ensuring that the heat exchanger has as little thermal resistance as possible, 
the effect of the convective thermal resistance on the total thermal resistance will be more pronounced, and so conclusions will be easier to draw.

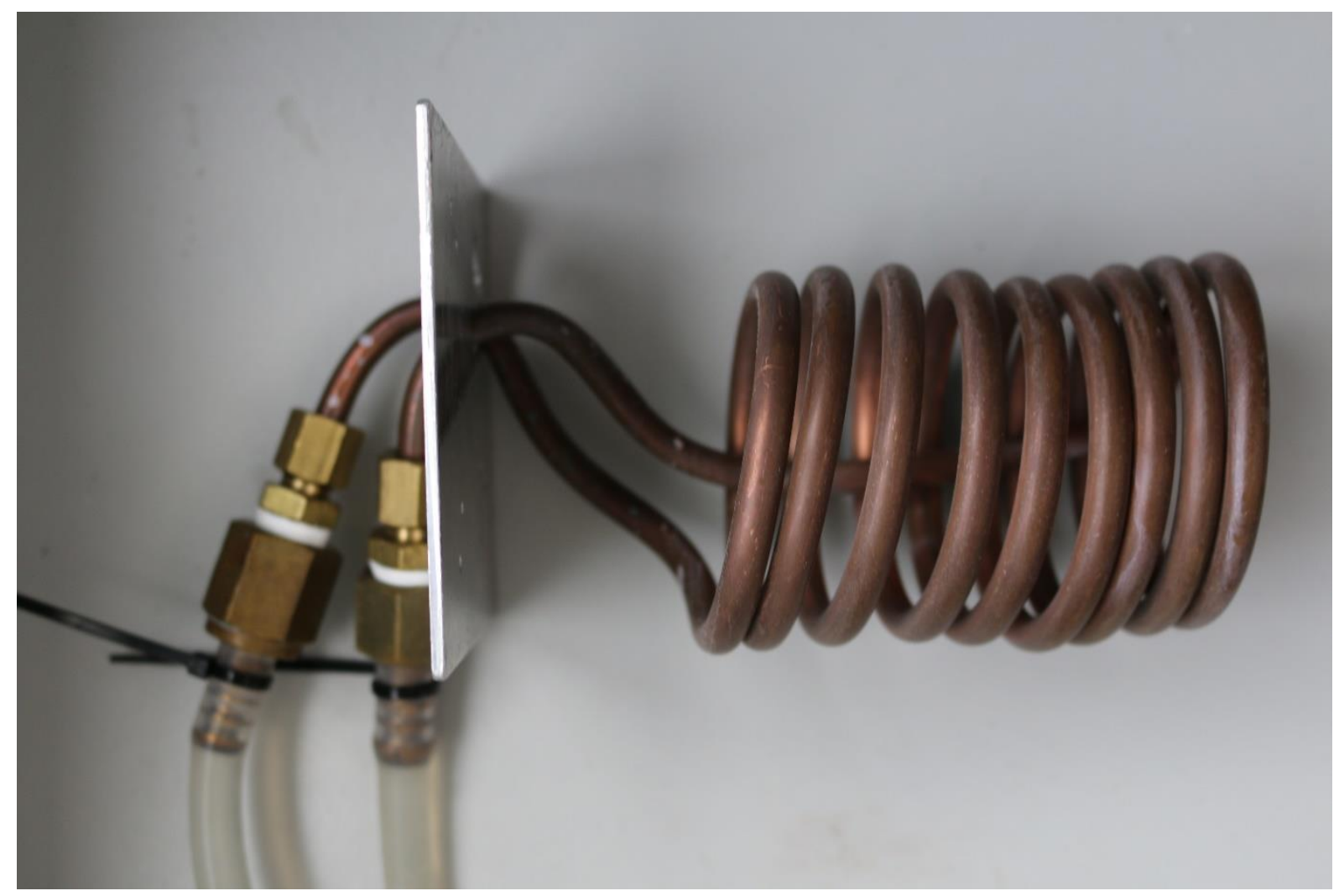

Figure 27: Heat exchange coil. The entirety of the tubing below the metal plate remains submerged in the cooling tank.

To characterise the capability of the heat exchanger, the cooling system had to be run. The flow rate was set to $0.4 \mathrm{l} / \mathrm{min}$, as this would allow the fluid to heat up significantly before leaving the chamber. The power input was increased until the heater temperature settled at $40^{\circ} \mathrm{C}$, and was then stepped up in stages of $5 \mathrm{~V}$ until the heater temperature settled at $100^{\circ} \mathrm{C}$. At each step, the temperatures of the fluid at the inlet and outlet of the heat exchanger were recorded. From here, the power $q$ being dissipated could be calculated via

$$
q=\Delta \mathrm{T}_{f} C_{w} \mu
$$

where $\Delta \mathrm{T}_{f}$ is the inlet to outlet temperature differential, $C_{w}$ is the specific heat capacity of water $(4.18 \mathrm{~J} / \mathrm{g} \cdot \mathrm{K}[17])$, and $\mu$ is the mass flow rate $(7 \mathrm{~g} / \mathrm{s})$. 
This power was then used to calculate the thermal resistance $\theta$ via equation (2.10), where $\Delta T$ is the difference in temperature between the fluid at the inlet and the coolant.

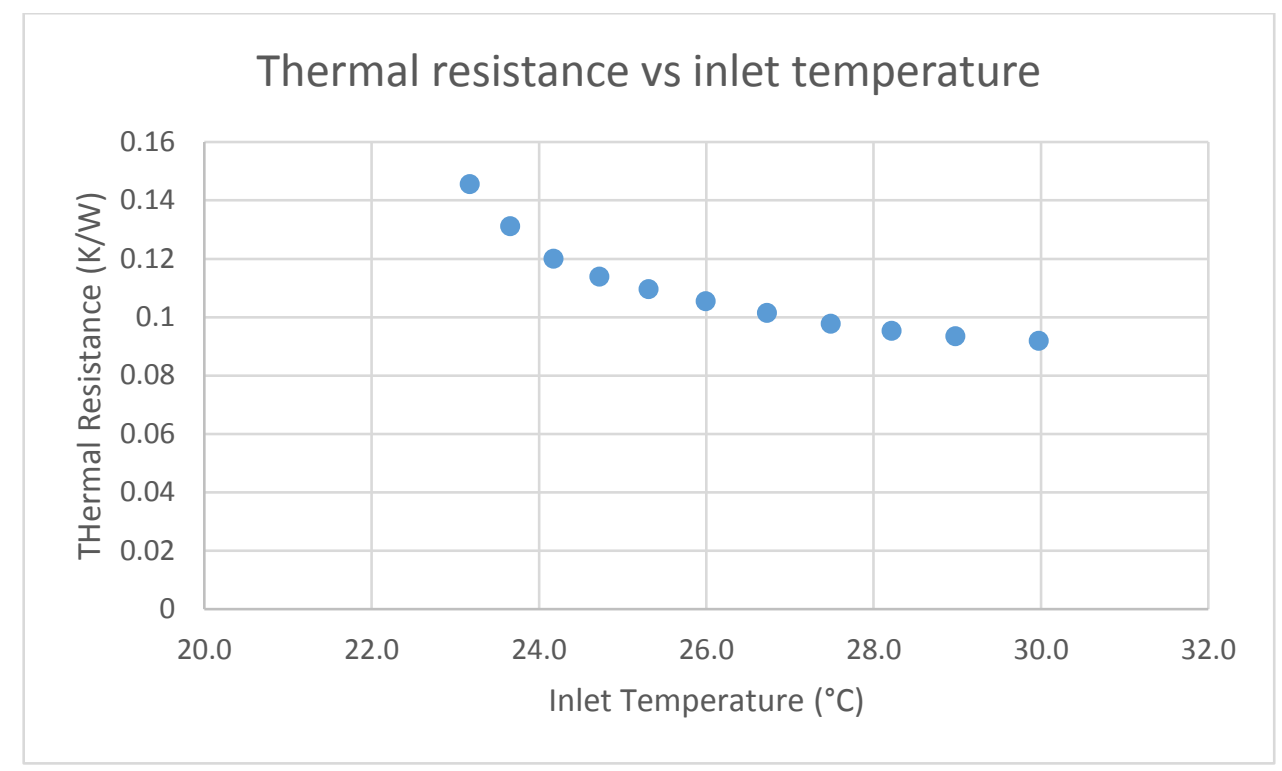

Figure 28: The heat exchanger's thermal resistance with respect to inlet fluid temperature.

The thermal resistance of the heat exchanger was found to decrease as the temperature of the inlet fluid increases, with an average thermal resistance over the values measured of $0.11 \mathrm{~K} / \mathrm{W}$.

\subsubsection{Reservoir and bubble trap}

The reservoir consists of a simple column with an inlet and outlet at the base connected between the heat exchanger and the pump. This provides excess liquid to ensure the inlet pipe is always filled with liquid. Air holes in the cap also allow the reservoir to remove any gases that form in the system due to degasification from the temperature change, or from the process of disconnecting and reconnecting the enclosure when switching the substrate. 


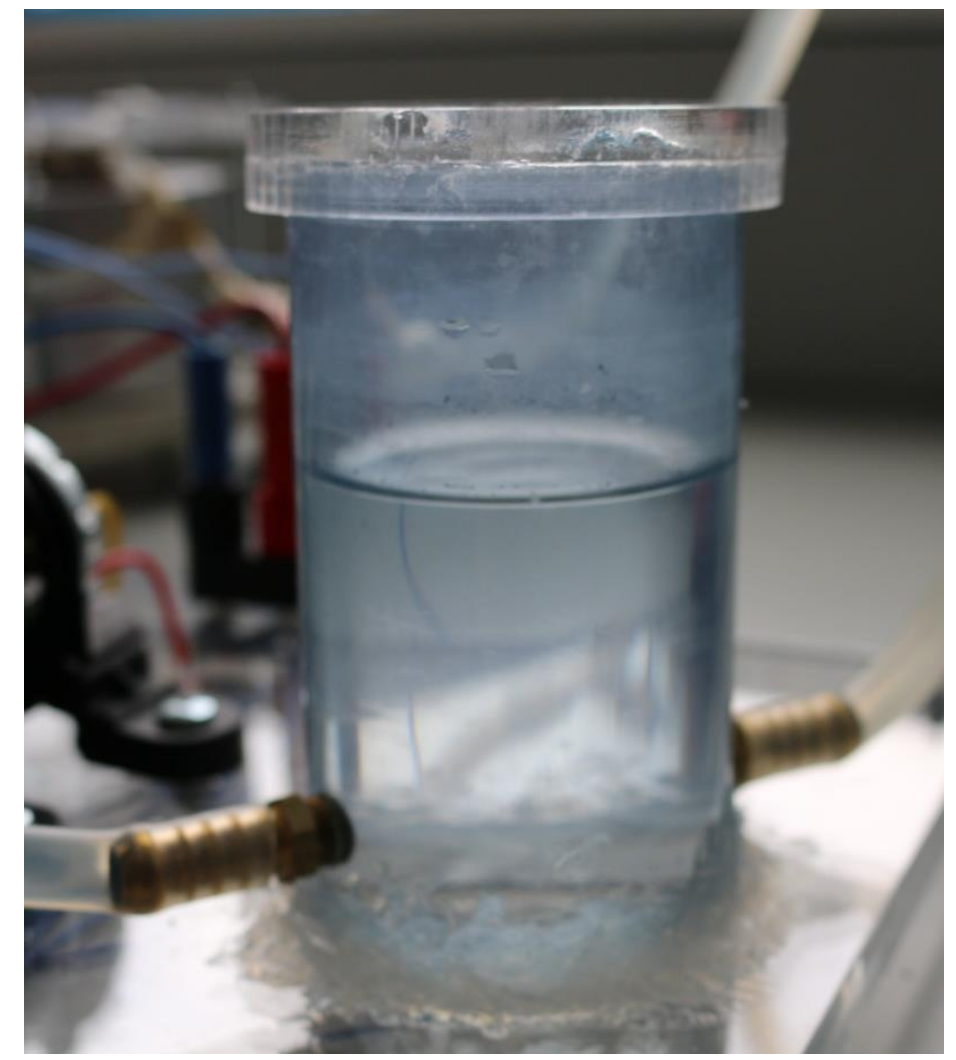

Figure 29: The reservoir and bubble trap.

\subsubsection{Tubing and connectors}

The tubing used to connect the pump, flow meter, thermocouple submerging connectors, reservoir, and heat exchanger is $1 / 8^{\text {th }}$ metric silicone tubing. Similar dimensioned brass connectors are attached to the reservoir, flow meter and heat exchanger, which are sealed to be liquid tight, while the pump and thermocouple submerging connectors have plastic connectors of the appropriate size built into them. $1 / 16^{\text {th }}$ metric silicone tubing connects the chamber and pressure sensor, and brass $1 / 8^{\text {th }}$ to $1 / 16^{\text {th }}$ connector adaptors are used to bridge between these two tubing sizes. The tubing is fitted over these connectors, and the connectors on the pump, flow meter and heat exchanger are secured with cable ties to prevent detachment at higher flow rates. These points were selected as they tended to detach at lower pressures than the enclosure. Because of the modular nature of the system, the enclosure cannot be tied in place, so connections that can withstand higher flow rates than the enclosure connections do not require securing.

Silicone tubing was used because of the design constraints of this project. The system needed to be designed on a budget, most of which was spent on sensors so, when possible, portions 
of the system were assembled using components which were readily available. However, silicone tubing also offered the ability to manoeuvre the different sections of the system if needed, which was an important quality to have in a prototype. Optimally, this tubing would be replaced with a more rigid material, so that much larger pressure differentials could be measured and to reduce the effects of ambient air pressure on the pressure sensing system.

\subsection{Sensing system}

The initial cooling system covered the functional goals of the system, but not the analytical goals. To this end, a system of sensors needed to be integrated into the system to facilitate the reading of important parameters.

The following were decided as the parameters to measure:

- Heat flux

- Substrate temperature

- Inlet fluid temperature

- Outlet fluid temperature

- Air temperature

- Flow rate

- Pressure differential

The heat flux and temperature measurements are measured using thermocouples. Thermocouples are single wire sensors, and so are simply installed at the position they need to measure. The two thermocouples in the coolant had to be put in submerging connectors, which are attached in series at the inlet and outlet of the chamber. The flow rate is measured by a flow meter, which is installed in series before the inlet. The pressure differential is measured by a differential pressure sensor, which is installed in parallel to the chamber. Both the submerging connectors and the flow meter have little hydraulic resistance. The pressure sensor has essentially infinite hydraulic resistance, so the effect it has on the hydraulic resistance of the system is also negligible. 


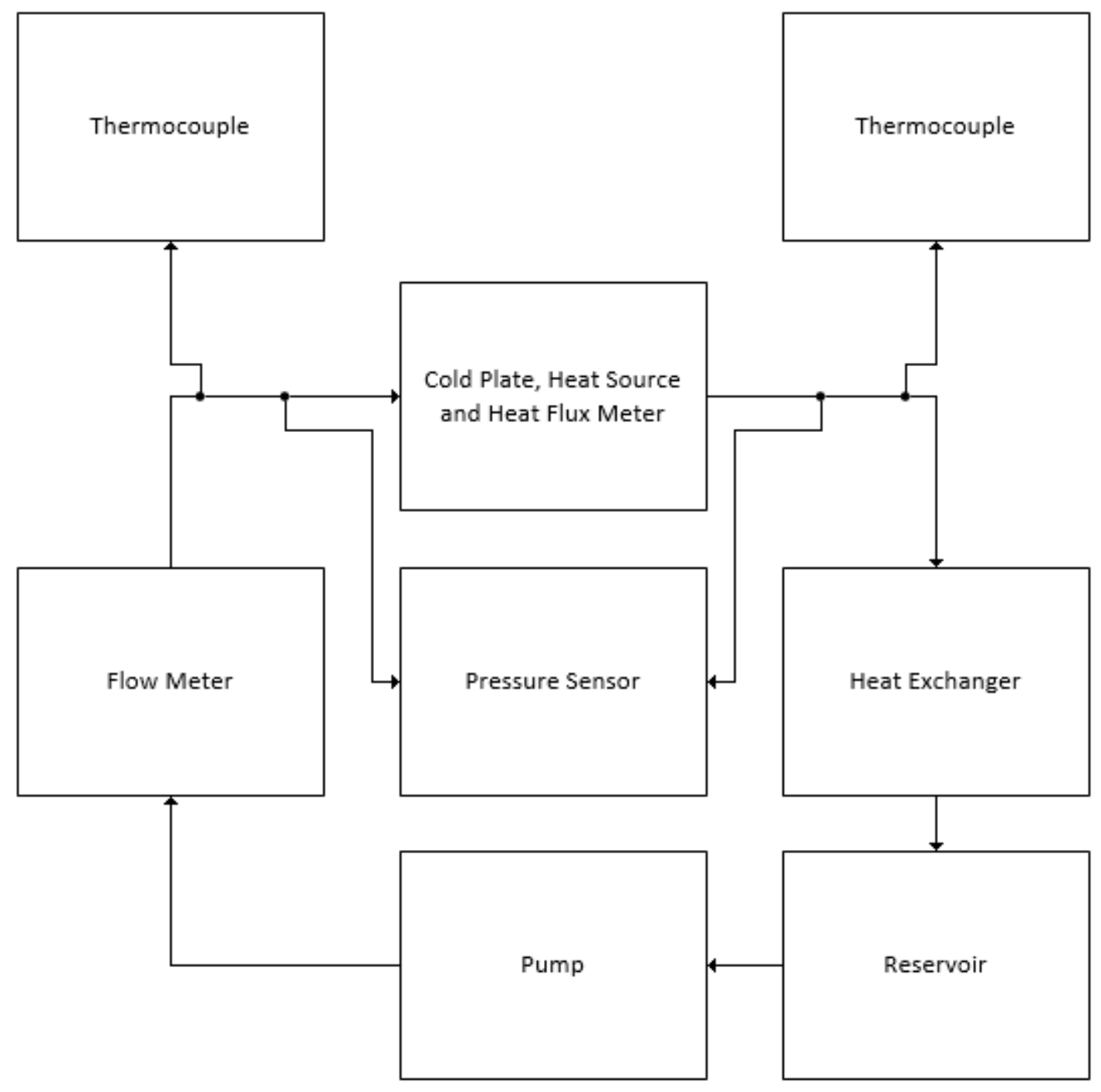

Figure 30: Block diagram of the cooling and sensing systems.

\subsubsection{Heat flux meter}

The most important parameter to measure is heat flux. This is because it is essential for calculating the thermal resistance, in this case calculated via

$$
\theta=\frac{\Delta T}{q^{\prime \prime} A}
$$

where $\Delta T$ is the temperature difference between the surface and the fluid, $A$ is the surface's area, and $q^{\prime \prime}$ is the heat flux through that surface. 
To measure heat flux, a simple heat flux meter was designed into the heat source detailed in section 3.1.1. The heat flux meter uses four thermocouples, one at the tip of the heating elements, a second $7 \mathrm{~mm}$ above this, then a third $5.5 \mathrm{~mm}$ above that, and the last $5.5 \mathrm{~mm}$ above that, just below the surface of the column. The difference in length of the first gap was due to mechanical constraints. These thermocouples measure three temperature differentials which can be used alongside the distances between the thermocouples and the thermal conductivity to calculate three heat fluxes via equation (2.1).

These heat fluxes are then averaged together to give a mean heat flux through the copper column. Because the thermal resistance around the column is significantly higher than the thermal resistance through it, the heat flux through the column should be relatively constant, so this value is used as an approximation of the heat flux at the surface/coolant interface.

$$
q^{\prime \prime}=\frac{k_{C u}}{3}\left(\frac{T_{1}-T_{2}}{d_{1}}+\frac{T_{2}-T_{3}}{d_{2}}+\frac{T_{3}-T_{4}}{d_{3}}\right)
$$

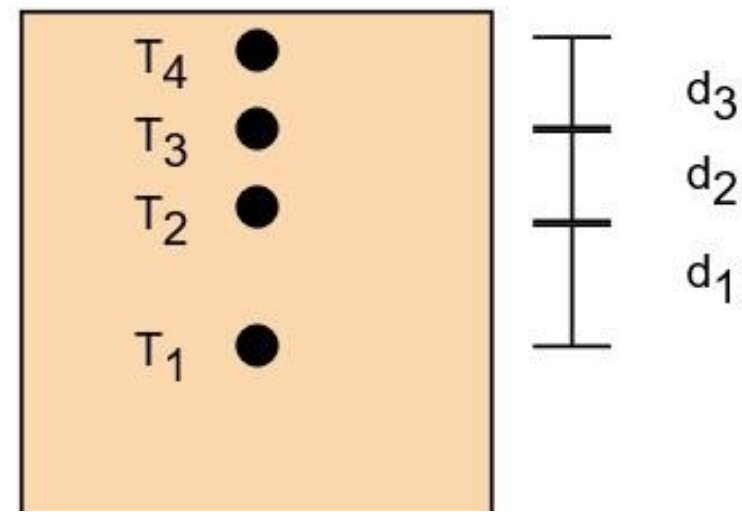

Figure 31: Full heat flux meter equation and diagram of setup. $T_{n}$ represents the temperature measured by a thermocouple at that point, and $d_{n}$ represents the distance between $T_{n}$ and $T_{n+1}$.

\subsubsection{Thermocouples}

The thermal resistance equation also communicates another important parameter, this being the temperature differential between the surface and the inflowing coolant. This requires both these temperatures to be known, so two additional thermocouples will be used to monitor these values. The outflowing coolant and air temperatures are also measured. These 
two temperatures are not used in any calculations but help provide context to the results in the event of incongruences.

To measure temperature, eight type-k thermocouples are used. These thermocouples are $0.5 \mathrm{~mm}$ and coated with a $0.25 \mathrm{~mm}$ thick polytetrafluoroethylene (PTFE) coating, allowing them to easily insert into the thermocouple holes detailed above. As with the interface between the heating column and the substrate, each thermocouple in contact with copper is coated in thermal interface material to ensure good thermal contact. The first four of these are positioned in the heat flux meter arrangement mentioned in section 3.2.3. The fifth, sixth, seventh and eighth are positioned in the thermocouple hole in the substrate, in the open air, and in submerging connectors on the inlet and outlet flows of the chamber, respectively.

The submerging connectors presented a challenge to design, as the PTFE coating has a hole at either end to let the wires pass through. The pressure inside the connector was sufficient to push the liquid up under the coating and into the instrument reading the thermocouples. This meant it was important to seal the ends of the thermocouples used in the submerging connectors. Applying hot melt adhesive (HMA) to the ends and surrounding this with heat shrink succeeded in solving this problem. These thermocouples were then inserted into Tjoint pipes in the perpendicular axis and sealed in with sealing caps and two-part silicone resin.

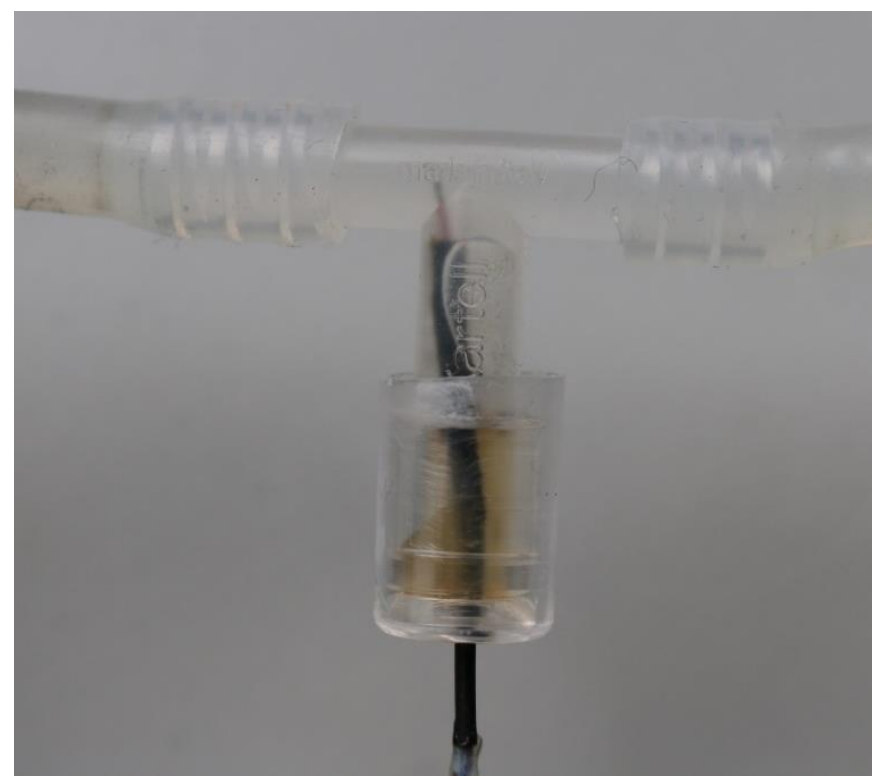

Figure 32: Thermocouple submerging connector. 
The thermocouples were calibrated by submerging them in a temperature-controlled bath alongside a previously calibrated PT100 temperature sensor. This bath was varied in temperature between $0^{\circ} \mathrm{C}$ and $100^{\circ} \mathrm{C}$. The PT100 was used to accurately measure the temperature of the bath at the exact position of the tip of the thermocouples, as the bath's average temperature by position had significant variance.

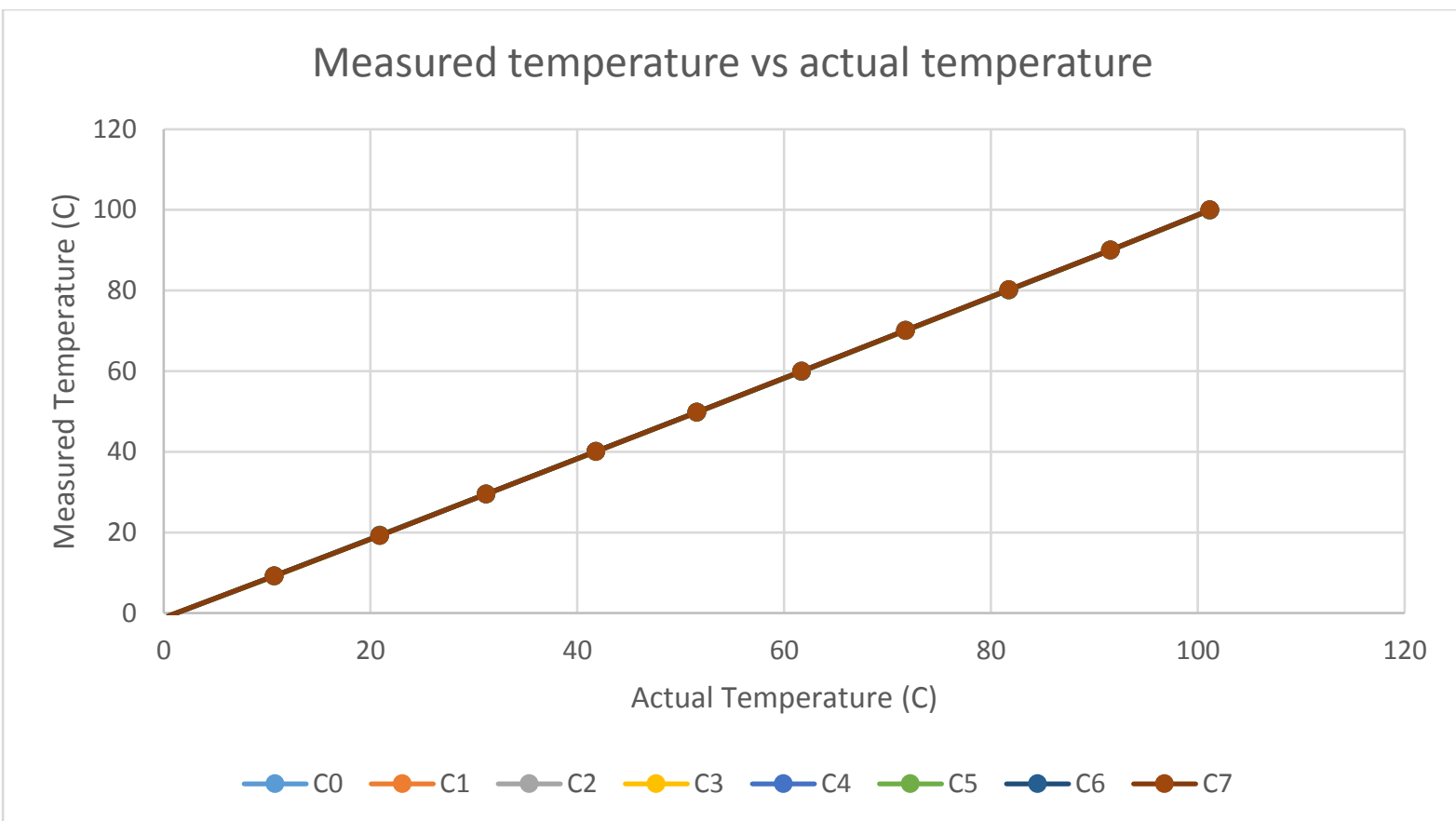

Figure 33: The thermocouple calibration results. Note that all eight thermocouples are depicted in this graph, but they are all directly behind one another.

\begin{tabular}{|c|c|c|c|c|c|c|c|c|}
\hline Channel & 0 & 1 & 2 & 3 & 4 & 5 & 6 & 7 \\
\hline Scaling & 1.0001 & 1.0002 & 1.0004 & 0.9999 & 0.9999 & 1.0007 & 1.000 & 0.9996 \\
\hline Offset & 1.5494 & 1.5626 & 1.5851 & 1.5946 & 1.5242 & 1.5364 & 1.5693 & 1.5681 \\
\hline
\end{tabular}

Table 4: The thermocouple calibration values.

The relationship between measured temperature and actual temperature is extremely linear, with only a slight offset. The error for a given measurement rated at $0.39^{\circ} \mathrm{C}$, and the fit of the linear models for each thermocouple was $R^{2}>0.99995$. This means that the data received from the thermocouples is extremely reliable. 


\subsubsection{Flow meter}

In addition to the thermal resistance, the hydraulic resistance is a desired statistic. As discussed in section 2.4, hydraulic resistance is one of the limiting factors when reducing characteristic length of a channel to decrease thermal resistance, and so it is important to monitor this value. Hydraulic resistance is calculated using equation (2.20). This means the flow rate and pressure differential both need to be measured.

To measure flow rate, an Omega FTB2003-c hall-effect flow meter was used [44]. The FTB2003-c outputs an oscillating digital output, with a frequency that increases with flow rate, and measures this via a rotating propeller on the inside of the sensor. Due to some resistance on the propeller, the flow meter can only output above a certain minimum flow rate, below which it will output a frequency of zero.

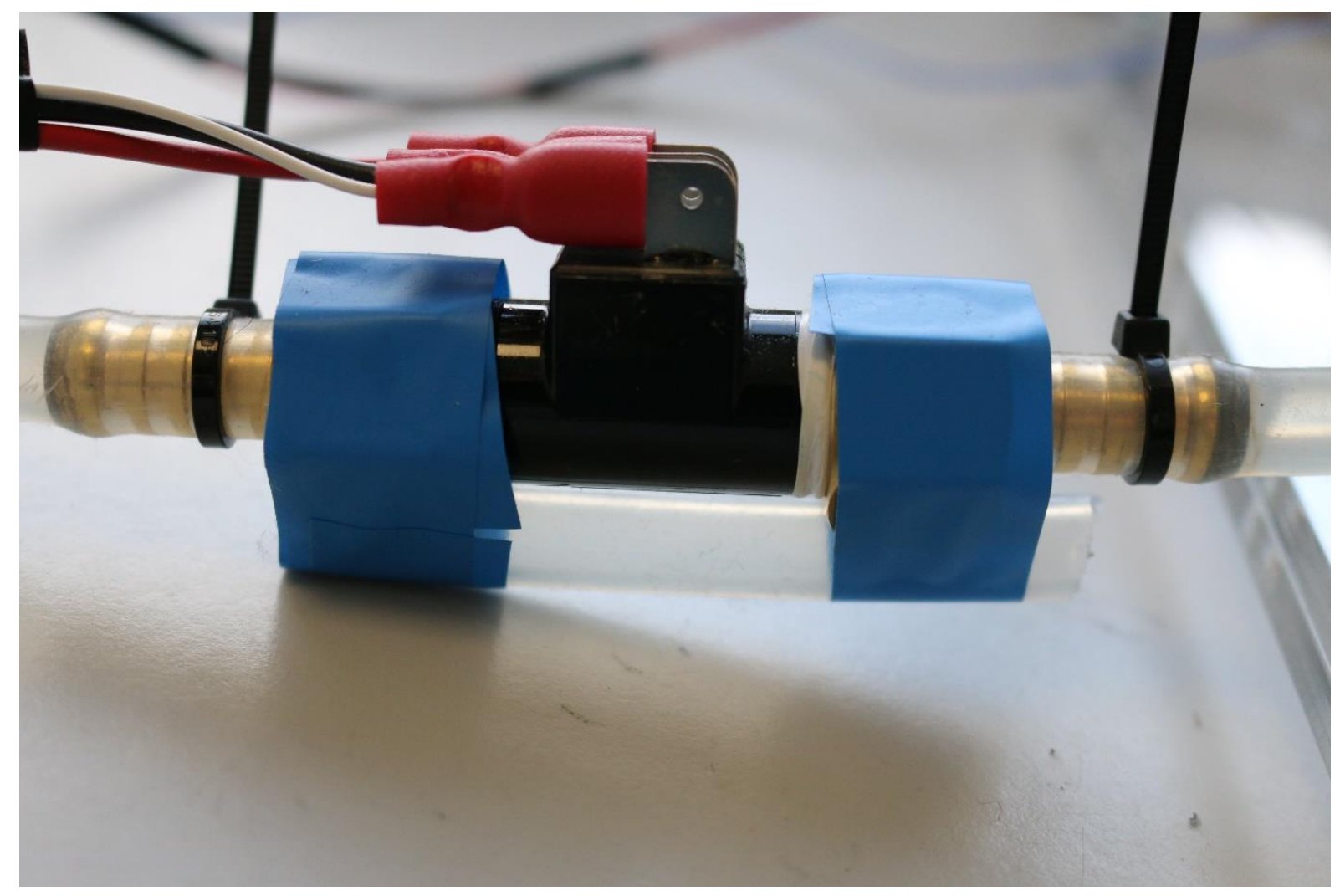

Figure 34: The Omega FTB2003-c hall-effect flow meter.

The flow meter was calibrated alongside the free flow characterisation of the pump, previously shown in Figure 26. While recording the mass displacement, the frequency of the 
flow meter was noted, and this frequency was then compared with the resultant flow rate calculations.

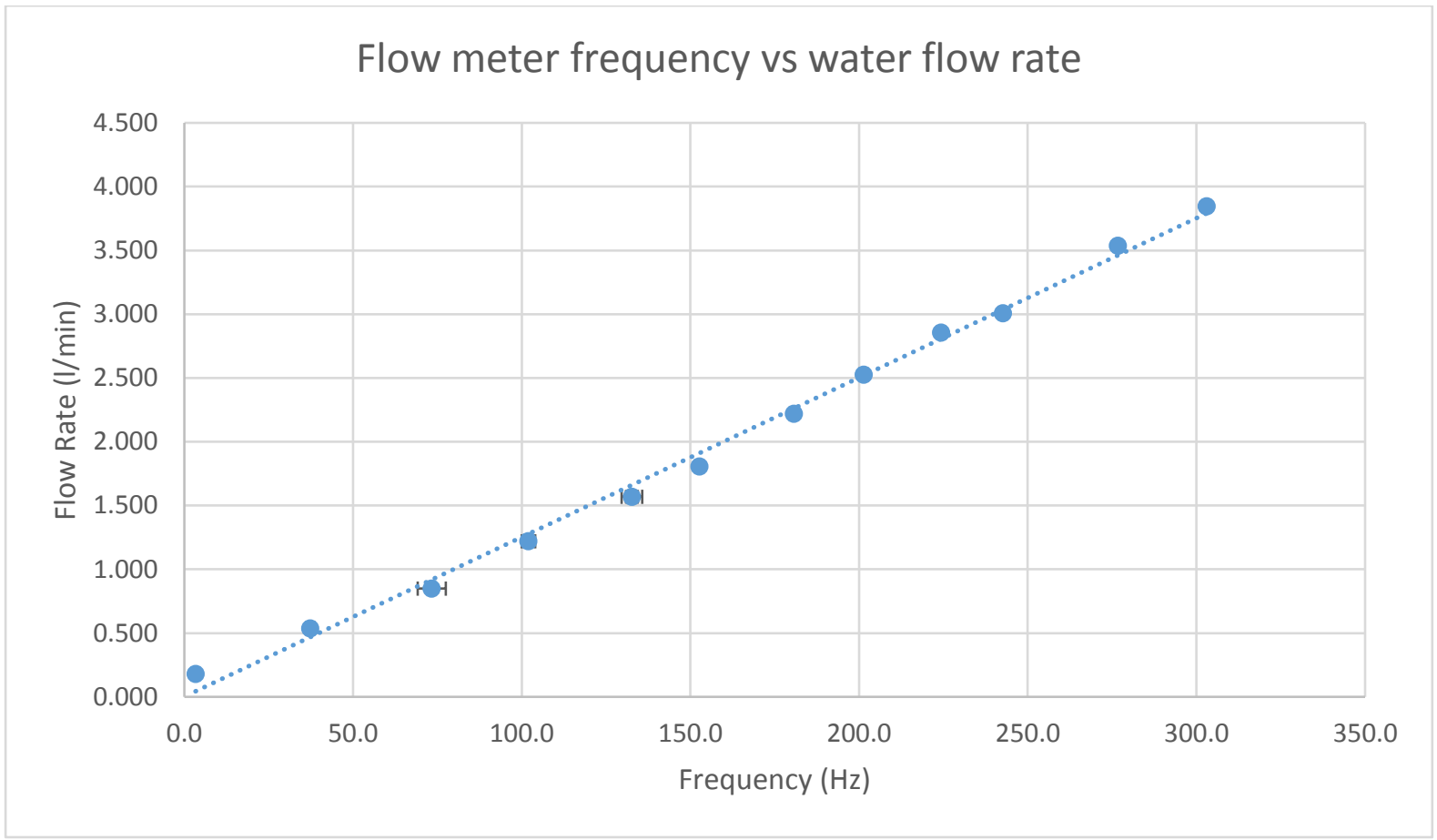

Figure 35: The flow meter calibration results.

\begin{tabular}{|c|c|}
\hline Flow rate $=($ Scaling $*$ frequency $)+$ offset. & Flow rate $(\mathrm{l} / \mathrm{min})$ \\
\hline Scaling & 0.0125 \\
\hline Offset & 0.0021 \\
\hline
\end{tabular}

Table 5: The flow meter calibration values.

The meter frequency output increases at an approximately linear rate over the range being measured. The error of a given measurement is about $0.03 \mathrm{l} / \mathrm{min}$, and the fit of the linear model is $R^{2}=0.9958$. This indicates that the flow data is sufficiently reliable, particularly at higher flow rates.

\subsubsection{Differential pressure sensor}

Pressure differential is the other statistic required to calculate hydraulic resistance.

To measure the pressure differential, an Omega PX26-030DV differential pressure sensor was used [45]. The PX26-030DV outputs a voltage based on this difference, and measures these pressures using a pair of diaphragms. 


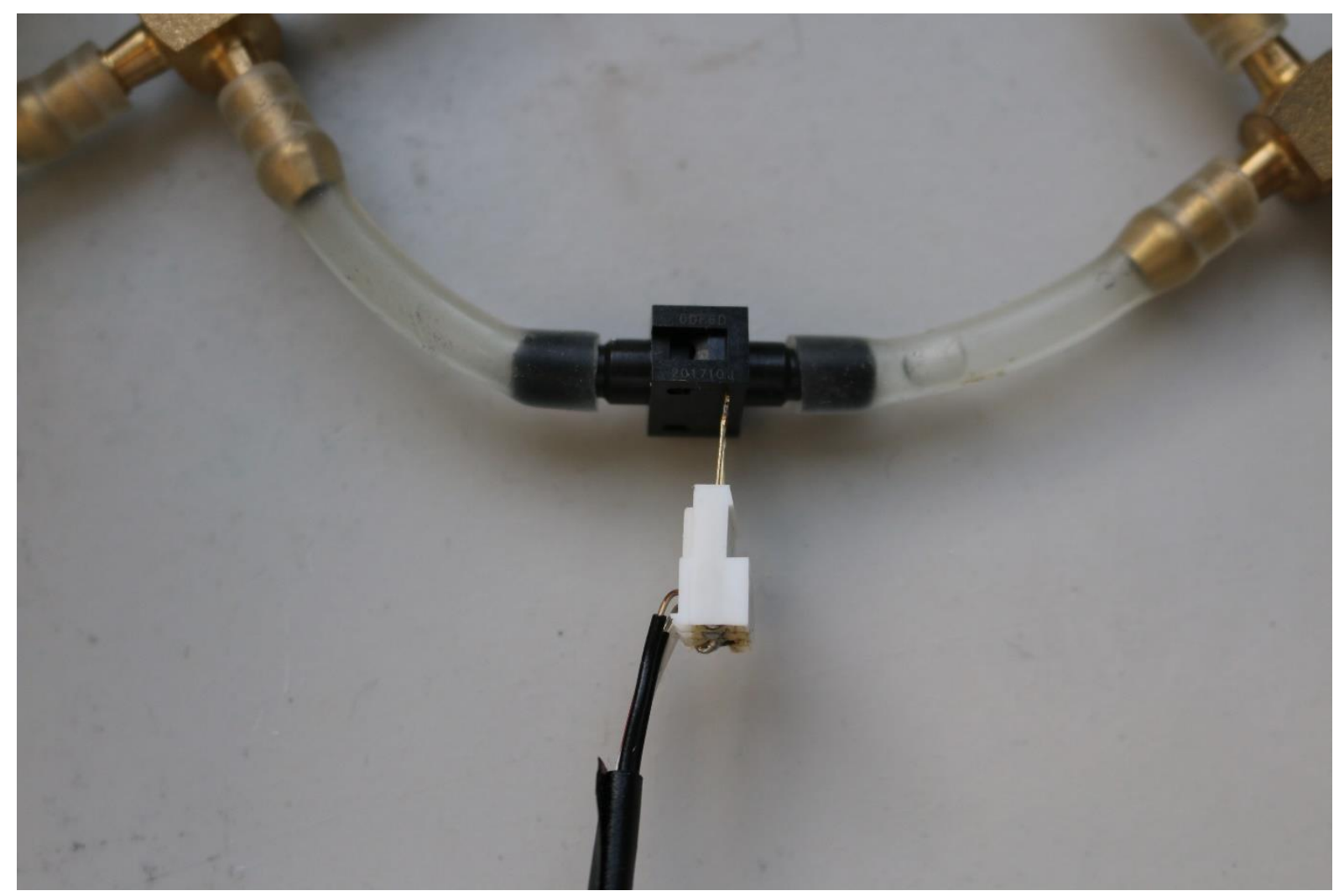

Figure 36: The Omega PX26-030DV differential pressure sensor.

Initially, a pair of PX26-005GV gage pressure sensors were used instead [45]. However, these failed to cover the required range, and issues with calibration were also present. Consequently, they were replaced. This is noteworthy however, as artefacts of their involvement are still present in the final system, as their presence had no negative consequences on the performance of the system.

The differential pressure sensor was calibrated using a pair of $\sim 60 \mathrm{ml}$ plastic syringes. The actual volume of the syringes was not exact, because an extra $0.5 \mathrm{ml}$ was present in the syringe which could not be expelled by the plunger. One syringe was filled to maximum capacity with air and attached to the front end of the pressure sensor, while the other was evacuated as much as possible and attached to the back end. By compressing the filled syringe, the pressure on the front end could be increased via Boyles law, which states

$$
P \propto \frac{1}{V}
$$


Similarly, by pulling on the plunger of the emptied syringe, the tiny volume that could not be expelled would expand, causing the pressure on the back end to decrease. By carefully controlling the volumes of the two syringes, a range of pressure differentials could be created. The pressure differentials were then calculated and compared to the output voltage of the pressure sensor.

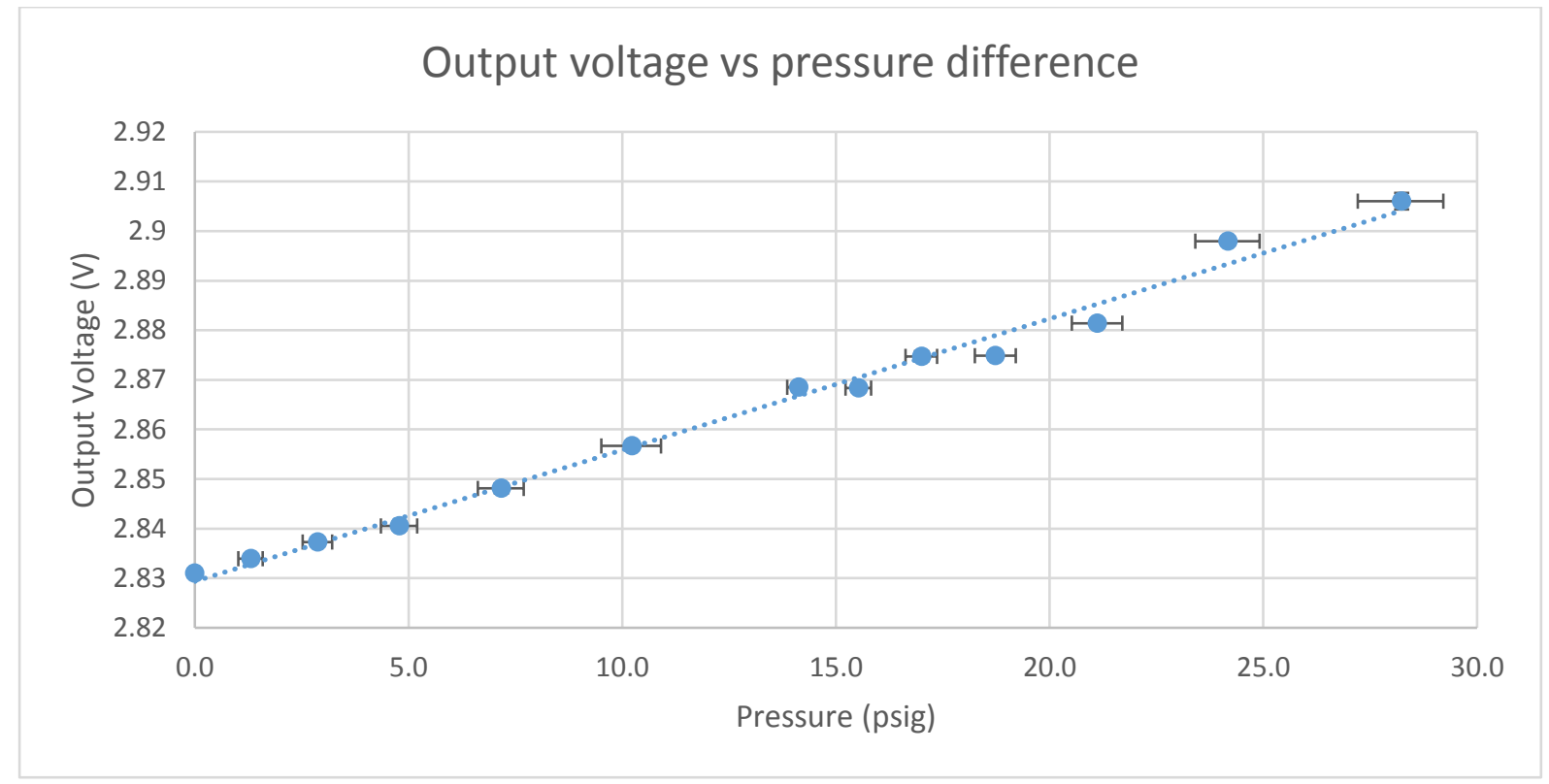

Figure 37: The pressure sensor calibration results.

\begin{tabular}{|c|c|}
\hline Pressure $=($ Scaling $*$ voltage $)+$ offset. & Pressure (PSIG) \\
\hline Scaling & 373.93 \\
\hline Offset & -1057.9 \\
\hline
\end{tabular}

Table 6: The pressure sensor calibration values.

While the differential pressure sensor still has an approximately linear relationship, there is a far greater margin of error than with the other sensors. The error associated with a given measurement varied significantly, but the maximum was around 0.65 PSIG. The fit of the linear model was $R^{2}=0.99$, and it is clear from the graph that the model is less appropriate at significantly large pressure differentials.

It was also found that the voltage offset shifts slightly from day to day. This is likely due to atmospheric changes altering the latent pressure on the sensor, as the tubing used in the system can be compressed. While this offset is a small amount when compared to the voltage 
being output, when the pressure is calculated the offset is as big as 1.5 PSIG, as the scaling factor is significantly large. This requires resetting at the beginning of each test.

Evidently, of the major test elements, the pressure sensor is the least reliable.

\subsection{System integration}

The sensors and heaters were connected to a computer to read their outputs and control the heater power. This section details the additional hardware used to connect these items to the computer, and the software used to read and process the data.

\subsubsection{Hardware}

The eight thermocouples are connected to the processing computer via a National Instruments cDAQ-9171 [46] and added NI-9212 c series temperature input module [47]. The cDAQ-9171 is a modular data acquisition (DAQ) system, and the temperature input module is the module specifically designed for reading thermocouples and resistance temperature detectors. It outputs via a USB, and can be read by the standard DAQmx LabVIEW palate VIs. The measurement sensitivity of this connection is $0.01^{\circ} \mathrm{C}$, with a maximum sample rate of 95 samples per second. The output from the DAQ to the computer is at $12 \mathrm{Mb} / \mathrm{s}$.

The flow and pressure sensors are connected via a National Instruments USB-6009 [48]. Unlike the specific modules of the CDAQ-9171, the USB-6009 is a general-purpose digital/analogue IO device, although it still outputs via a USB, and can still be read by the standard DAQmx LabVIEW palate VIs. The pressure sensor is connected to one of the analogue inputs, which have a resolution of 13 bits. The flow meter is attached to the event counter input, which has a resolution of 32 bits and a maximum input frequency of $5 \mathrm{MHZ}$. The output from the DAQ to the computer is at $12 \mathrm{Mb} / \mathrm{s}$. Small circuit boards were designed to connect the sensors to the DAQ, as they could not attach directly. As mentioned in section 3.2.2, originally the differential pressure sensor was a pair of gauge pressure sensors, and an artefact of this is clear in the pressure adapted circuit. 

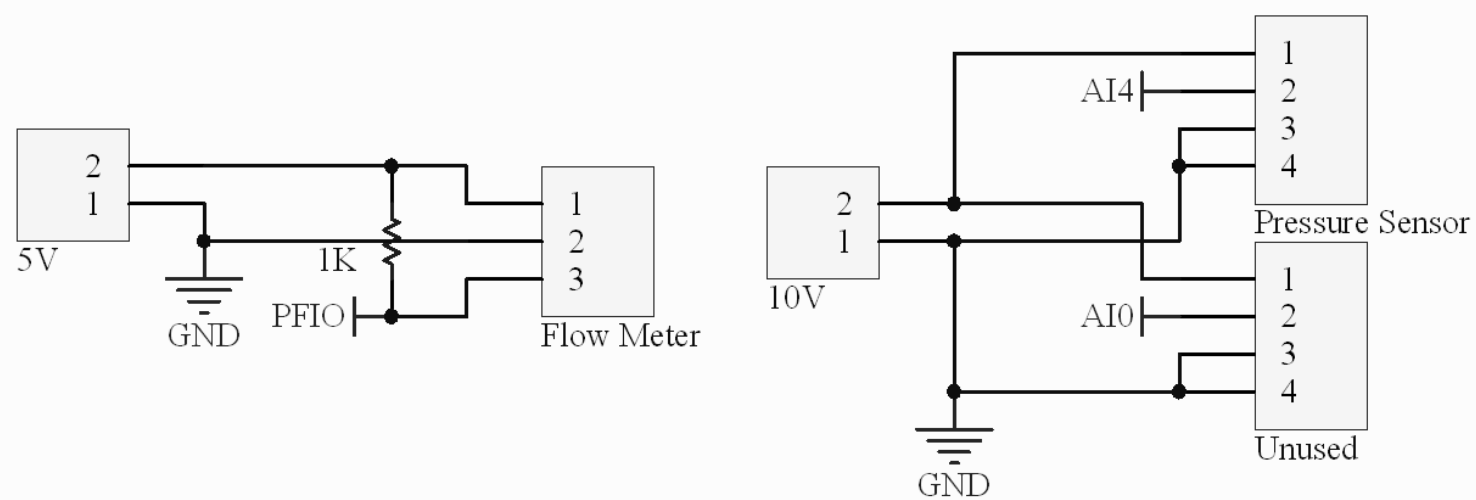

Figure 38: Circuit diagram of the flow adapter (left) and pressure adapter (right).

The power to the heater was supplied via a GW INSTEK PSW 160-7.2 multi-range DC power supply which connects to the processing computer via a USB [49]. This power supply can be controlled by the PSW LabVIEW palate VIs and can be set to output a precise voltage or current by adjusting the maximum allowed values for each.

The power to the pressure sensors and the pump was supplied via a Topward 6303 dual tracking DC power supply [50]. The voltage for each output can be set independently but must be set manually via potentiometers on the face of the power supply. While this might seem like a downside, as the precision to which the values can be set will have a higher variance than the GW INSTEK power supply, this is not a significant issue. Unlike the heater voltages, which must be varied repeatedly throughout testing, the voltages supplied to the pressure sensors and the pump will almost always be kept at the same value, so this variance will have little opportunity to present itself.

\subsubsection{Software}

The program running the test system is written in LabVIEW. Its goals were to take the data being supplied to it by the GW INSTEK power supply and the National Instruments DAQs and output relevant information on the front end, as well as control the output of the GW INSTEK power supply.

The program consists of three stages. It begins with a small initialization stage, where the program connects to the external systems and assigns space for some updating variables. The bulk of the program is then dedicated to a loop block, where the data is retrieved, processed, 
and presented on the front end. To finish the program, there is a brief finalization stage, where connections are closed properly, and errors are processed. This stage only happens when the loop is stopped, which is done manually when closing the program.

\subsubsection{Heater control code}

The heater code needed to have inputs for a serial configuration and VISA resource name to connect to the GW INSTEK power supply. It also required desired maximum voltage and current values to set the power supply output, and an enable switch to turn the heater off when heating is not desired. The outputs needed to include the actual voltage and current being provided by the power supply. It also needed to include alerts when the desired maximum voltage or current exceeded the rated maximum voltage and current of the power supply ( $160 \mathrm{~V}$ and $7.2 \mathrm{~A}$, respectively), as well as when the actual power provided by the power supply exceeded the rated maximum power output (360 W). The code uses the PSW palate provided by GW INSTEK for use with their PSW series power supplies.

The code begins by connecting to the power supply using the "PSW Initialize" VI, with the inputs from the serial configuration and VISA resource name variables.

This is then passed inside the loop and into the "PSW Configure VI Level" VI, which takes the maximum voltage and current values and increases the output power until either of these values meets their maximum. It also checks that both of these values are below their respective rated values, and if they are not, it turns on the alert and disables the heater. The configured data is then passed to the "PSW Configure Output" VI to turn on the output on the power supply side, which requires the enable to be on and the desired voltage and current to be below their rated values to continue. This is then passed to the "PSW Read Output" VI to acquire the actual output voltage and power on the VI side, and these values are passed to the actual voltage and current doubles. They are also converted into the current output power, which is checked against the rated maximum output power and an alert is raised if it exceeds this value. Unlike the voltage and current exceptions, this cannot disable the power supply, as it is checking the actual output of the power supply, so it is already on by this point. 
Finally, the data stream is passed out of the loop where the output is disabled, the desired voltage and current are set to 0 , the connection is closed, and errors from the stream are checked.

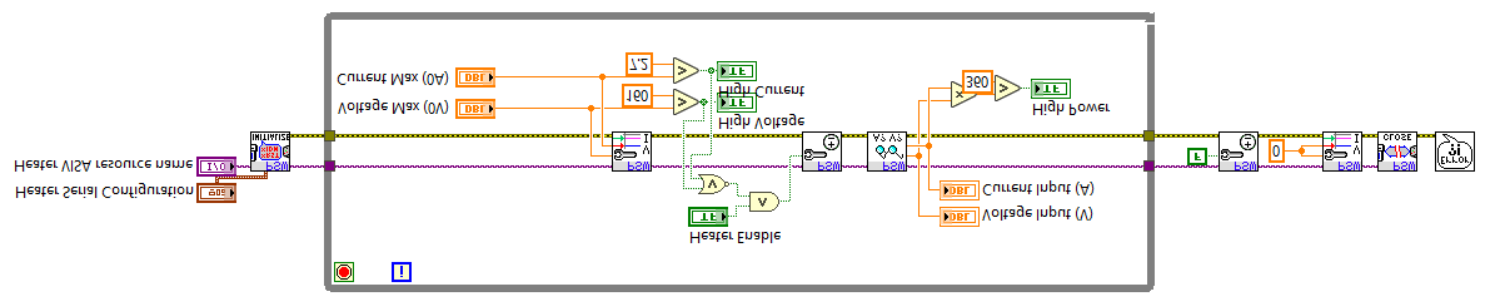

Figure 39: The heater control code.

\subsubsection{Flow meter code}

The flow meter code needed to take an oscillating input signal and convert that to a frequency, then into a flow rate. It needed to output the flow rate, and it was decided that outputting the frequency was also reasonable.

The code begins by connecting to the ctro input on the DAQ using the "DAQmx Create Channel" VI set to count falling edges. This is then passed to the "DAQmx Start Task" VI to begin data acquisition, before passing into the loop. Additionally, an integer is initialised to keep track of the last count, and the "Get Date/Time In Seconds" VI is used to find the arbitrary starting time for this initial count, and these are both passed into the loop as well.

The data stream is passed into the "DAQmx Read" VI, which outputs the current number of oscillations from the DAQ. This number is subtracted from the current count, outputting the number of oscillations that have occurred during the last iteration, and is also used as the count value in the next iteration of the loop. Meanwhile, the current time for this loop is found, and the difference between this time and the last recorded time is calculated. The number of oscillations is divided by the time taken for those oscillations to take place, giving the frequency of oscillation in Hertz. The calibration results from section 3.2.3 are then used to convert this frequency to flow rate in $1 / \mathrm{min}$ as desired.

Finally, the data stream is passed out of the loop and into the "DAQmx Clear Task" VI to stop the process. 


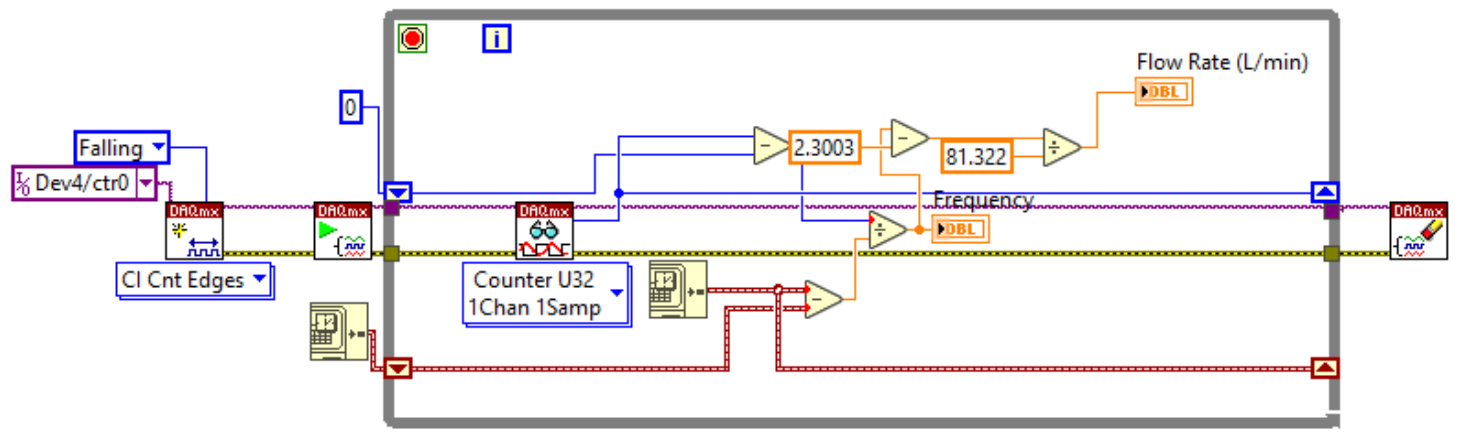

Figure 40: The flow meter code.

\subsubsection{Pressure sensor code}

As mentioned prior, originally two pressure sensors were used, and the code was designed to meet this specification. However, since the code now reflects the use of a single pressure sensor, this section will discuss the design as though it was always created this way.

The pressure sensor code was required to take a voltage from the pressure sensor and convert it into a pressure value as an output. It also needed to know the frequencies required for sampling and displaying. These values were shared with the thermocouple data and were defined by the needs of that section of code. A maximum sampling frequency existed at around $50 \mathrm{~Hz}$, above which the computer being used could not process the requests fast enough, so this was set to $50 \mathrm{~Hz}$. The display frequency was the number of data points displayed per second, and so controlled the number of samples being averaged to make a data point. As temperature tends to meander, a high display frequency is unnecessary, so this was set to $2 \mathrm{~Hz}$.

The code begins with connecting to one of the analogue voltage inputs of the $D A Q$, measuring in the ground-referenced single-ended (RSE) mode. This data stream is then passed to the "DAQmx Timing" VI, which sets up the sampling to proceed continuously at the desired sampling frequency. This is then passed to the "DAQmx Start Task" VI to begin sampling, and then passed into the loop.

The data stream is then passed into the "DAQmx Read" VI, which outputs an N-by-1 twodimensional array, where $\mathrm{N}$ is the sampling frequency divided by the display frequency. This array is then converted into a one-dimensional array of length $\mathrm{N}$, and then averaged to create 
a single voltage data point. The calibration results from section 3.2.4 are then used to convert this voltage into a pressure in PSIG as desired.

Finally, the data stream is passed out of the loop and into the "DAQmx Clear Task" VI to stop the process.

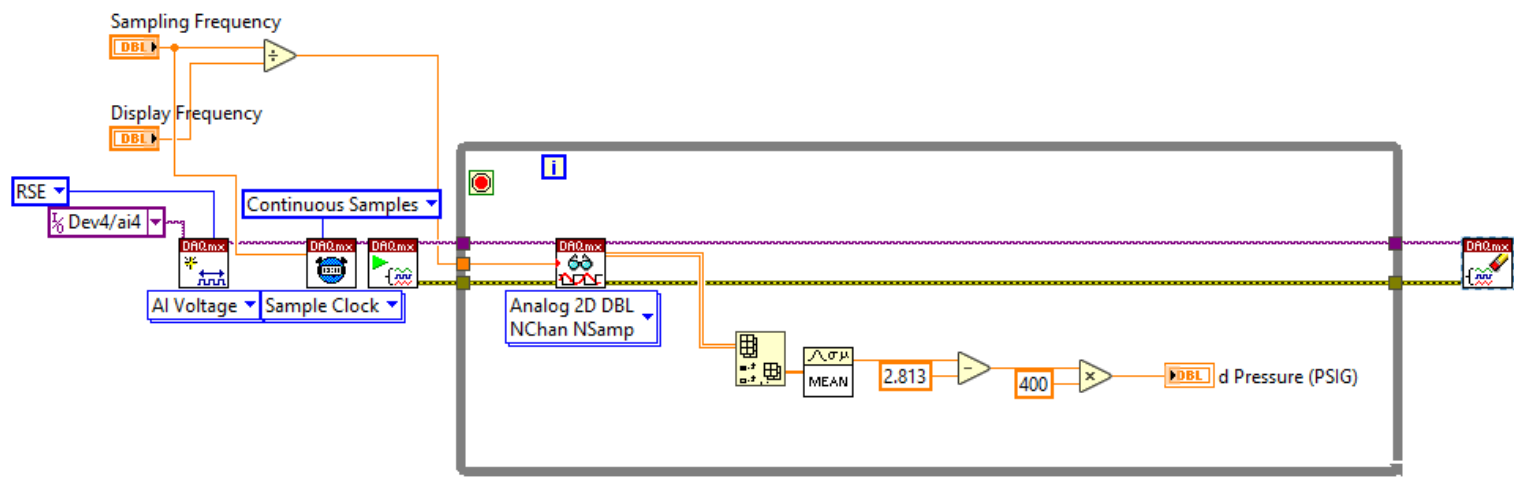

Figure 41: The pressure sensor code.

\subsubsection{Thermocouple code}

The thermocouple code needed to take data from eight thermocouples and adjust them to account for instrumentation errors before outputting them. It also needed to know the frequencies required for sampling and displaying, as discussed prior.

Each of the eight thermocouples was accessed by a "DAQmx Create Channel" VI, set to measure analogue k-type thermocouples with built-in cold-junction compensation (CJC). This data stream is then passed to the "DAQmx Timing" VI, which sets up the sampling to proceed continuously at the desired sampling frequency. This is then passed to the "DAQmx Start Task" VI to begin sampling, and then passed into the loop.

The data stream is then passed into the "DAQmx Read" VI, which outputs an N-by-8 twodimensional array, where $\mathrm{N}$ is the sampling frequency divided by the display frequency. This is then broken into eight one-dimensional arrays of length $\mathrm{N}$, and each of these arrays is averaged, giving a single value for each thermocouple. This value is adjusted by the rates found in section 3.2.2 and passed to the outputs. 
Finally, the data stream is passed out of the loop and into the "DAQmx Clear Task" VI to stop the process.

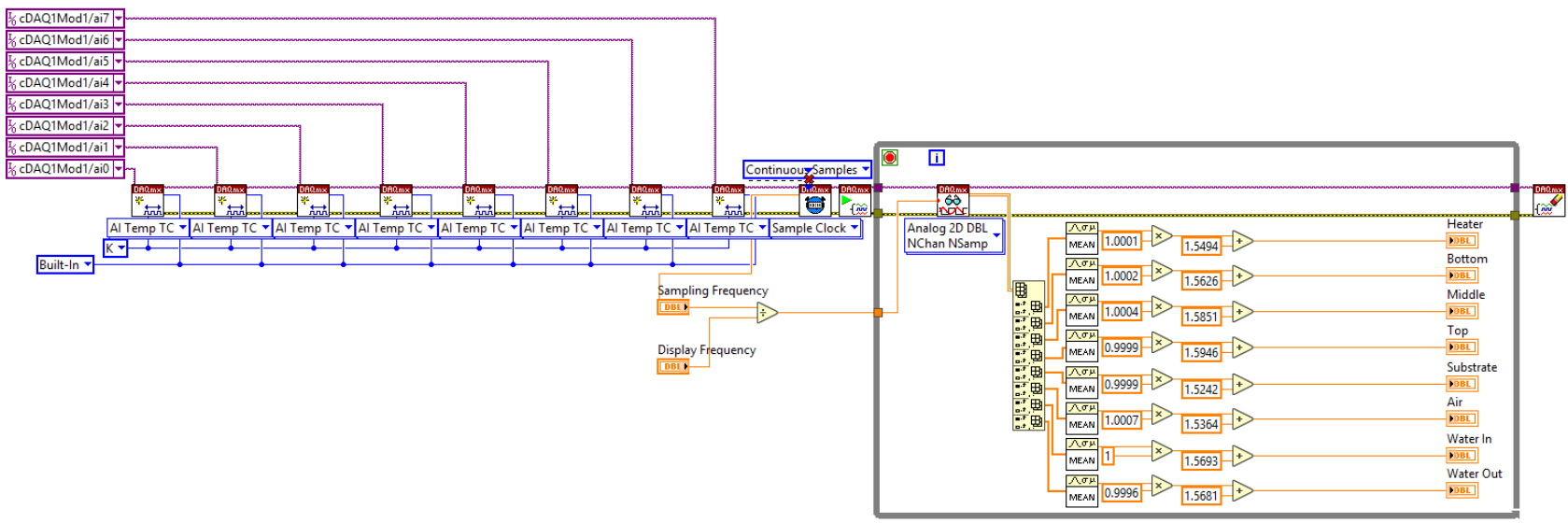

Figure 42: The thermocouple code.

\subsubsection{Processing code and the front end}

The processing code needed to take the outputs of each other section of code as inputs, and output a plot of all of the data, as well as calculate important statistics like the thermal resistance. Ultimately, it was decided that the outputs would include the following:

- Averages of each of the input statistics

- Standard deviation of the temperature statistics for use in identifying when the temperature is considered stable

- The heat flux

- The approximate power loss at the substrate surface

- The loss of power due to air convection

- The thermal and hydraulic resistances

The heat flux and approximate power loss required an additional three inputs

- The distance over which heat flux is measured

- The thermal conductivity of the heating column

- Cross-sectional area of the heating column 
Additionally, one final alert was added, which goes high if the power loss due to air convection goes negative. This would imply that more power is being lost by the system than is being supplied, which is not a physically possible steady state condition, so there must be an error occurring. Realistically, this alert should only be raised if the power input is reduced and the system has yet to cool down.

Before the loop begins, an array of size $\mathrm{N}$-by-13 is initialized, where $\mathrm{N}$ is the display frequency multiplied by the length of time over which the test should take place. In the tests run in this project, this value was set to 150 seconds, as this gave a total of 300 data points for processing. The array is initialized with null values, so that when graphing this beginning stage the graph will still scale to the data, as this allows any problems with testing to be more readily visible.

Inside the loop, the temperature, heater voltage and current, pressure and flow inputs are all collated in a one-dimensional array of length 13. This is amended to the end of the initialized array, before being output to the plot, but also to a feedback node. This feedback node passes the array into a splitter which separates it into 13 one-dimensional arrays of length $\mathrm{N}$, and each of these arrays is then rotated backwards one place such that the oldest data point of each is now at the end of each array. These are then recombined into one $\mathrm{N}$-by-13 and passed back into the amending function, where the oldest data points are overwritten by the new data again. It should be noted that the eleventh value in the final code is tied to zero instead of an input. This is an artefact of the pressure sensor change mentioned previously, as this was used as one of the pressure inputs initially.

Additionally, before recombination, the mean of each of the one-dimensional arrays is found, as well as the standard deviation of the thermocouple arrays. The heat flux is found by measuring the difference in temperature between each pair of adjacent thermocouples in the heating column, multiplying by the thermal conductivity of said column (in this case, $k_{C u}=$ 399), and dividing by the distance between those thermocouples (5.5 $\mathrm{mm}$ for C1-C2 and C2$\mathrm{C} 3$, and 7 for $\mathrm{CO}-\mathrm{C} 1$ ) before averaging. Multiplying the heat flux by the area of the column gives an approximate value for heat lost at the substrate surface, and the difference between this loss and the input power gives the loss due to convection. The sign of this value is checked 
and, if it is negative, an alert is raised. Finally, the pressure is converted from PSIG to kilopascals, and then divided by the flow rate to find the hydraulic resistance in $\mathrm{kPa} \cdot \mathrm{min} / \mathrm{l}$.

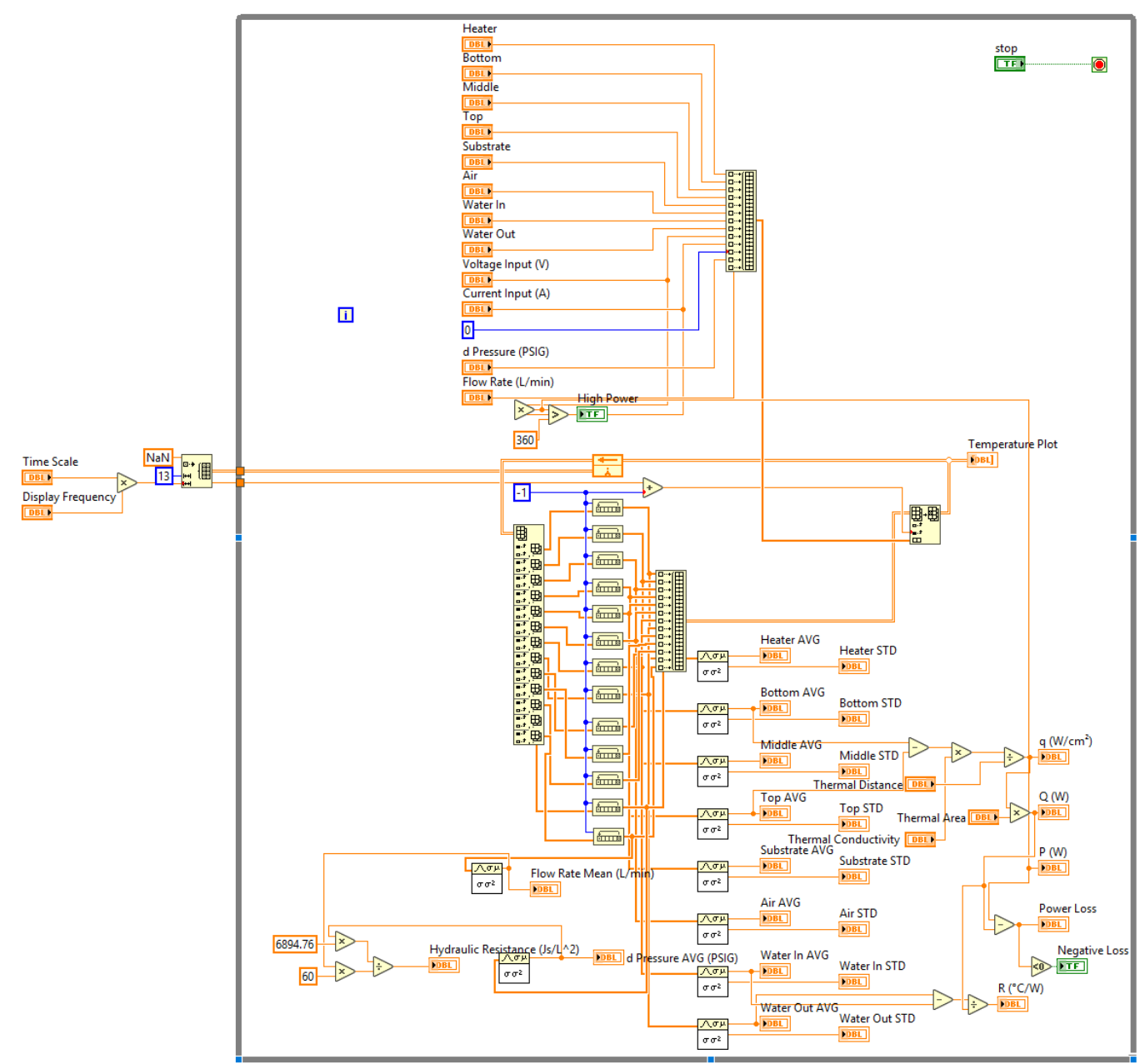

Figure 43: The processing code.

The front end needed to display all of the outputs of each block of code, including the graph containing the raw data. The front end was designed so that all of the relevant data would fit on one screen width of the computer chosen to run it, with the graph taking up the left half of the screen. The remaining data was separated into sections, with the timing values in the top left, the temperature data in top centre-left, the heater connection details in top centreright, the heater controls in bottom right, the pressure, flow rate and resistances in the top right and the heat flux and power data in the bottom left.

The graph technically contains data from all thirteen sources. However, during operation only the first five thermocouples are actually plotted. The rest of the information is input into the 
graph so that the data can be exported just by requesting the "export" option in the graph toolbar.

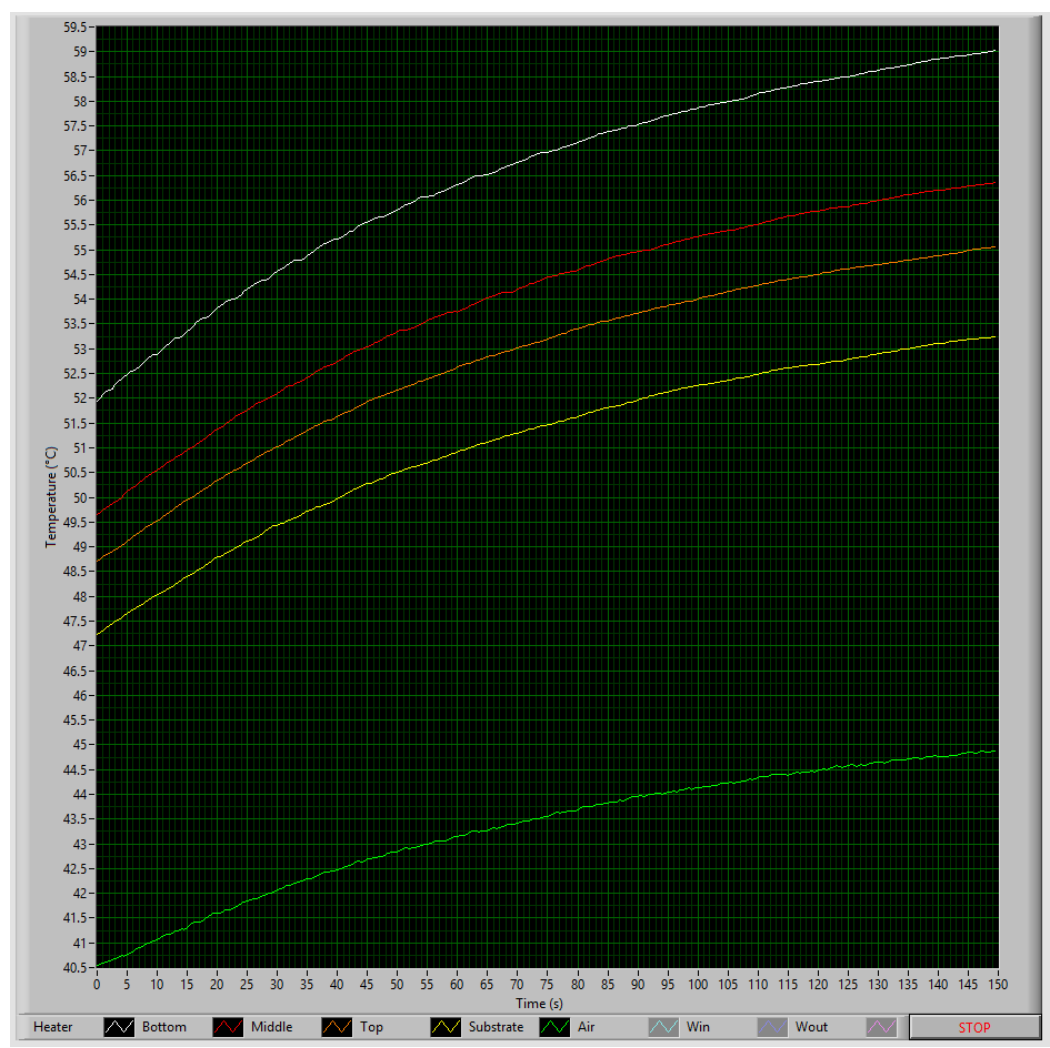

Figure 44: The front end graphing panel during operation.

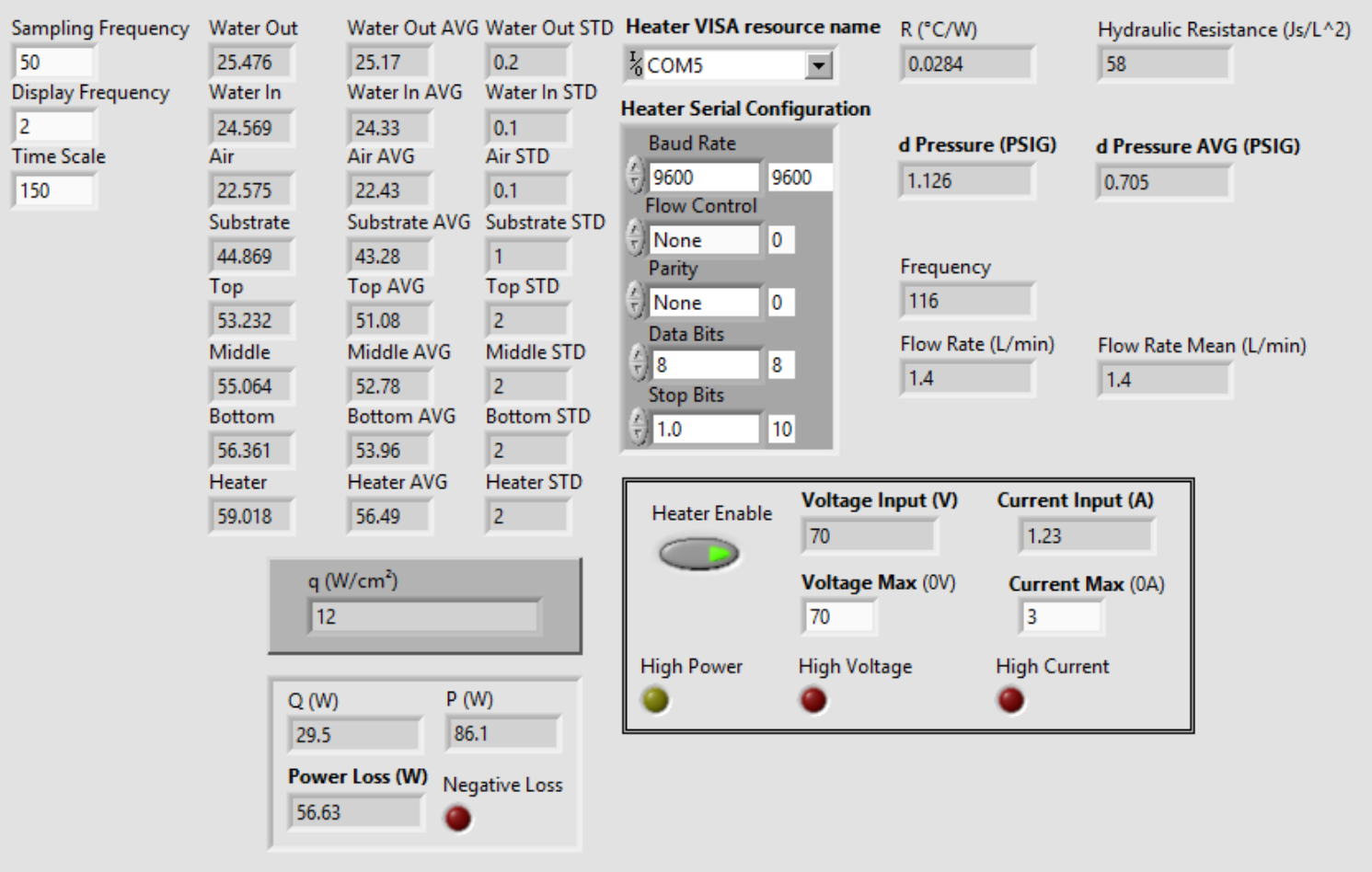

Figure 45: The front end data panel during operation. 


\subsection{Test procedure}

The test procedure takes a few hours to complete, with variance depending on the exact characteristics of the test being run. The standard procedure is as follows.

To begin, turn on the power supplies and coolant tank, pre-set the values in the LabVIEW program to the numbers detailed in section 3.3.2, and run the program. Set the input voltage to the pressure sensors to $10 \mathrm{~V}$ and change the input voltage to the pump until the desired flow rate is found.

Set the maximum current on the power supply section of the front end to $3 A$, and the maximum voltage to $5 \mathrm{~V}$, before enabling the heater. Slowly increase the input voltage in stages of $5 \mathrm{~V}$ until the heater temperature settles at $40^{\circ} \mathrm{C}$.

Now, let the temperatures of the first five thermocouples settle as much as possible. Right click on the graph and export the data. Record in the name of the file the input voltage of the pump, as this detail is not recorded by the system.

Now, in steps of five volts, increase the input voltage to the heater and repeat the previous step, until the heating temperature reaches $100^{\circ} \mathrm{C}$.

Using the same calculation methods mentioned in section 3.3.2.5, the heat flux, power loss, thermal resistance and hydraulic resistance can be calculated. Averaging the resistances with respect to temperature will provide the thermal and hydraulic resistances of the substrate being tested under the parameters chosen. 


\section{Fabrication and properties of porous and structured}

\section{surfaces}

The test system is a key component of the project, as previously documented in chapter 3. However, the test system is not the only experimental component of the project. In this chapter, the details of fabricating the FCS surfaces and other structured surfaces will be covered. The methodology of fabricating the FCS surfaces and measuring their contact angles will be detailed in section 4.1. Section 4.2 will cover key results noted during the fabrication process, particularly the effect of certain initial conditions on the contact angle of the surface being created. Finally, section 4.3 will cover the fabrication processes of the other surfaces used in the experiments.

\subsection{Methodology}

FCS surfaces are the primary form of porous microchannels being analysed in the project. This is because they are comparatively easy to fabricate in the lab, allowing for many varied surfaces to be fabricated during the project. However, their fabrication process is lengthy, taking several stages and multiple days to complete. The process will be outlined in this section, alongside the design and usage of the contact angle measurement system used to analyse their wettability.

\subsubsection{Fabrication of FCS surfaces}

The fabrication process begins with the creation of a water-based solution containing known concentrations of both silver and polyvinyl alcohol (PVA). Immediately before applying to the substrate, the solution is mixed using a vortex mixer, and then homogenized using an ultrasonic disrupter. Once the solution is homogenous, the substrate is placed on a scale, and the scale is zeroed. A Teflon ring is positioned around the tip of the substrate to help contain the fluid. The solution is applied with a pipette directly onto the surface and must be done carefully to avoid introducing air bubbles into the solution. The weight of the solution applied is recorded, and then the substrate is carefully moved to the surface freezer. 
The surface freezer consists of three layers. The bottom layer is a heat exchanger, which is fed fluid from a circulating bath set to $-15^{\circ} \mathrm{C}$. The middle layer consists of a four-square of thermoelectric Peltier cooling elements, connected in series and powered by an external power supply. These are positioned such that the hot side of the Peltier elements is cooled by the heat exchanger. The top layer is a copper disk, attached to the cold side of the Peltier elements, with a thermocouple hole attached to monitor the temperature at the surface. Plastic sealing surrounds the top two layers so that only the copper, the thermocouple hole, and the power cables for the Peltier elements are accessible.

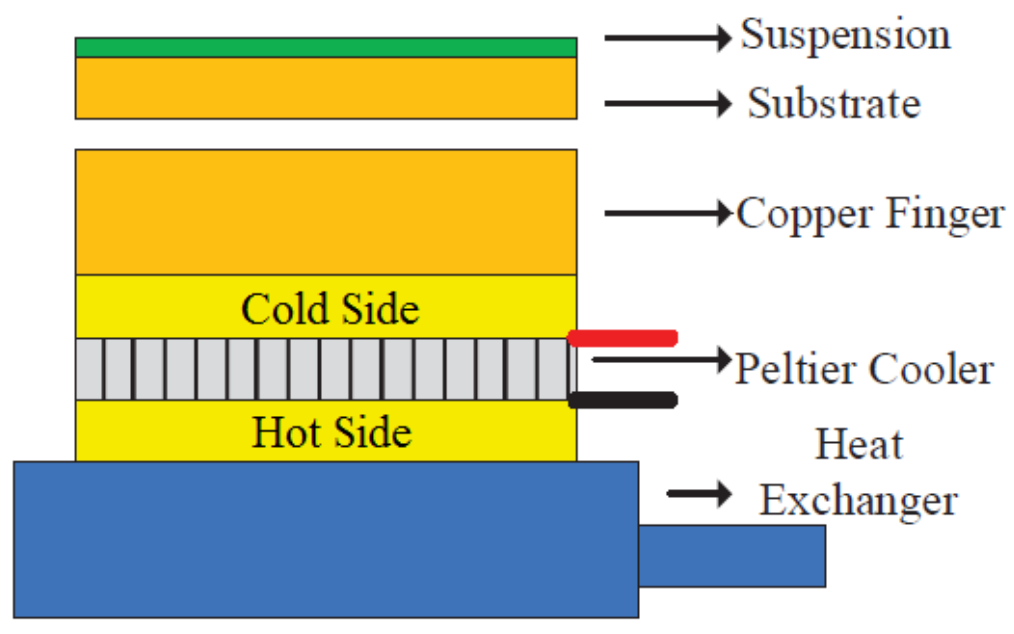

Figure 46: Surface freezer cross-section.

When freezing the silver solution, the cooling element input voltage is initially set to $-6 \mathrm{~V}$ such that the surface temperature is just above $0^{\circ} \mathrm{C}$. The substrate is placed on the surface with a thin plastic spacer to prevent the substrate from getting stuck to the surface after freezing. The voltage is then flipped to $+6 \mathrm{~V}$, and then raised periodically until it reaches $+15 \mathrm{~V}$, letting the temperature of the surface reach at least $-25^{\circ} \mathrm{C}$. This is left for a period of fifteen minutes, and then the substrate is separated and put into a freezer set to $-18^{\circ} \mathrm{C}$ to maintain the crystal structure that has formed. 


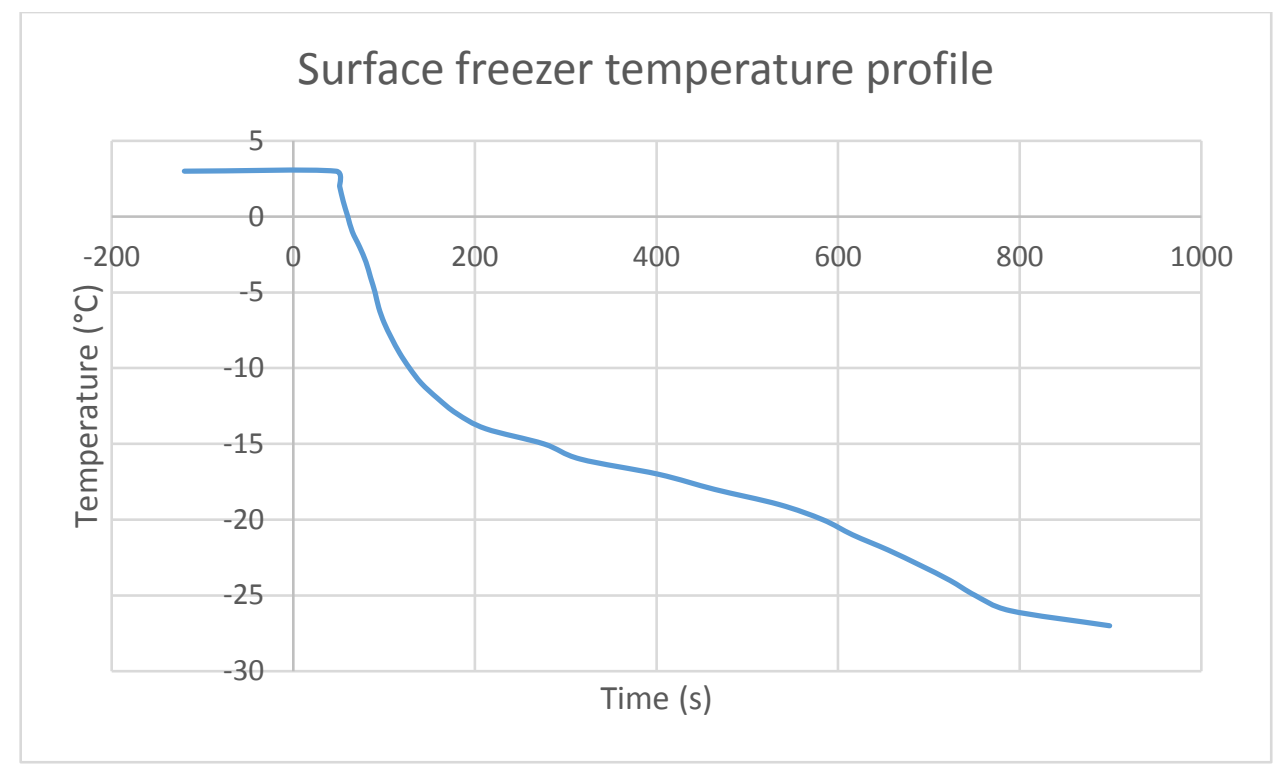

Figure 47: The temperature profile of the freezing process. Voltage was switched from $-6 \mathrm{~V}$ to $+6 \mathrm{~V}$ after 45 seconds. The periodic raising of the voltage is what results in the strange shape of the temperature curve.

After twelve hours, the surface is transported to a vacuum freeze dryer to remove the ice crystals via sublimation, preventing any water from destroying the structures formed during freezing. They are dried for at least 72 hours, before being transferred to a tube furnace for sintering.

The sintering process requires that the tube furnace atmosphere to be replaced with argon to prevent oxidation during heating. The temperature in the furnace is slowly raised to around $300^{\circ} \mathrm{C}$, before being left to heat for four hours. This is much lower than the melting point of silver but is significantly higher than the pyrolysis temperature for PVA. This breaks down the polymer, leaving some hydrocarbons and releasing various gasses, which are removed by the cycling argon.

Finally, after being removed from the surface, the samples are rinsed in boiling DI water. This step was introduced to the process after previous research noted that the FCS surfaces changed properties after being used in hot, wet environments [39]. It is suspected that this process is removing any excess PVA and components of PVA pyrolysis that may be left on the surface. However, this has yet to be confirmed, and the only known purpose of this process is to establish the properties of the surface before it is being used in tests. 


\subsubsection{Contact angle measurement}

Contact angle has been shown to affect heat transfer properties, with higher contact angles correlating with lower heat transfer [51]. As other aspects of the silver surfaces such as their precise characteristic length are difficult to identify, the contact angle was used so that a variable existed to distinguish between the silver surfaces beyond their fabrication parameters. As mentioned in section 2.4.4, contact angle is found my measuring the angle formed between the surface in question and the edge of the droplet.

To measure contact angle, a simple acrylic frame was constructed to house a Digitech QC319 digital microscope [52]. This frame positions the camera in front of a block on which the substrate is placed, such that the surface is slightly below the direct line of sight of the camera. A light box was constructed from black acrylic to remove outside light sources and make measurements easier to distinguish, and the part of the light box that lies behind the substrate from the camera's perspective was made from a matte material to reduce glare from the microscope's internal lighting.

Direct line of sight was avoided for two reasons. Firstly, the silver surfaces are rough, and direct line of sight would mean roughness at the edges could obscure droplets at the centre. Secondly, thicker silver surfaces would move the surface level up, and this would prevent the measurement system from working if the system was level with the surface of the base substrate.

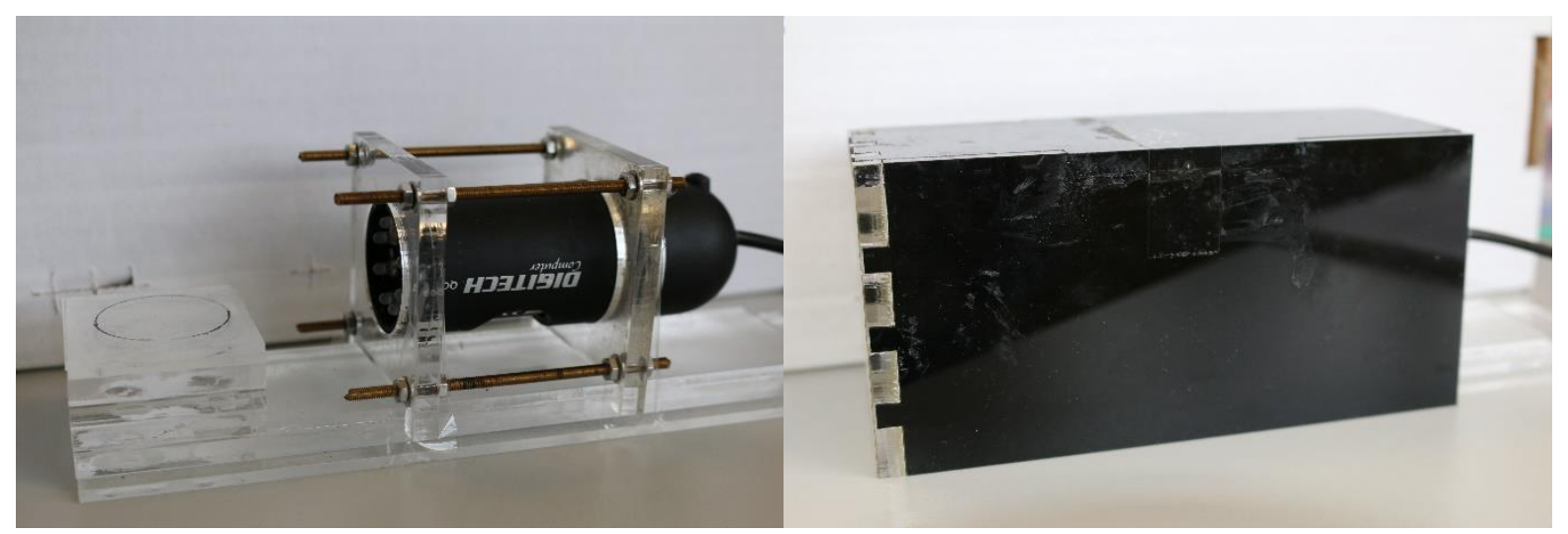

Figure 48: Contact angle measurement device exposed (left) and under light box (right). 


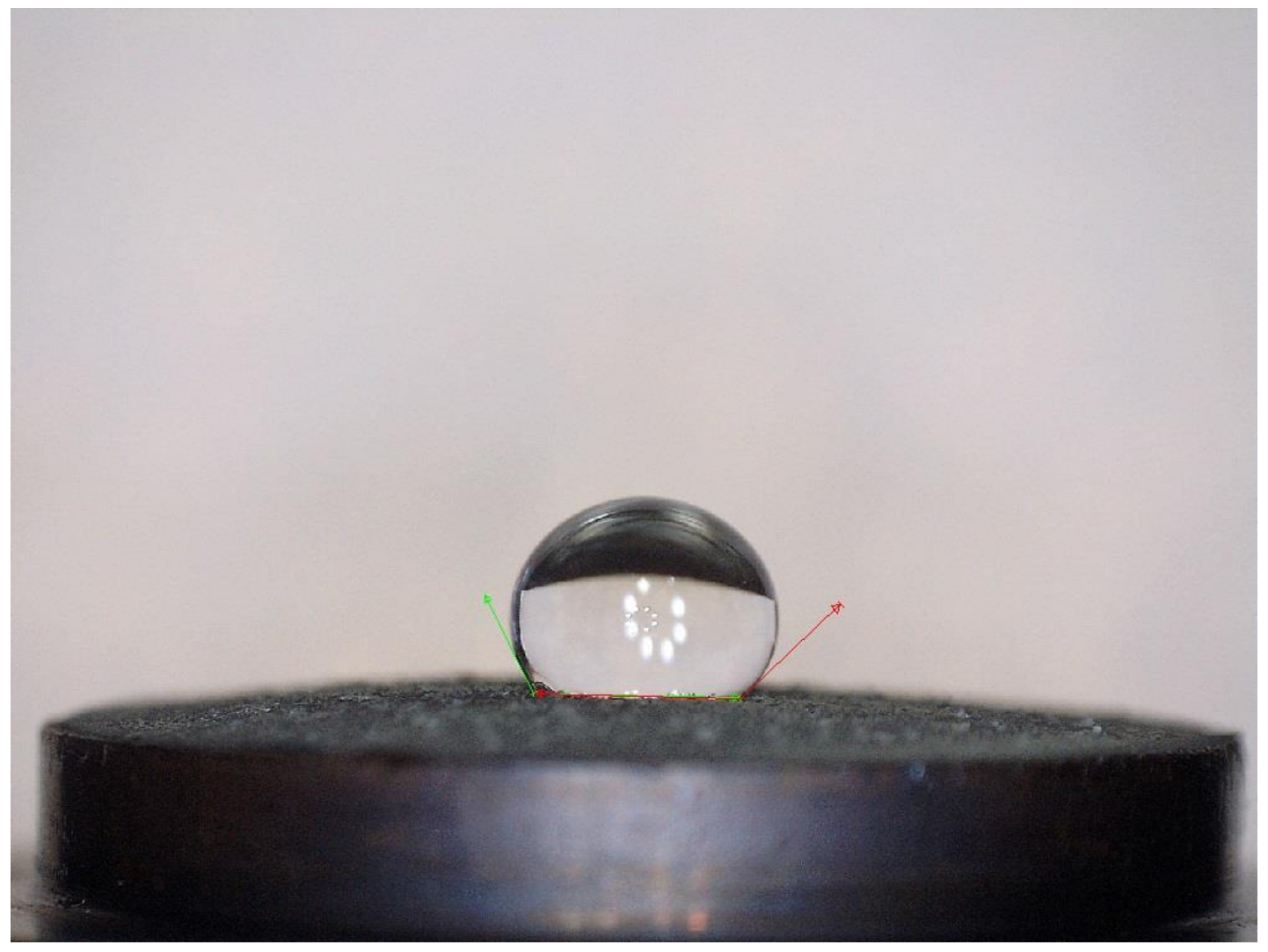

Figure 49: An example of a contact angle measurement. This sample is exhibiting slight hydrophobicity.

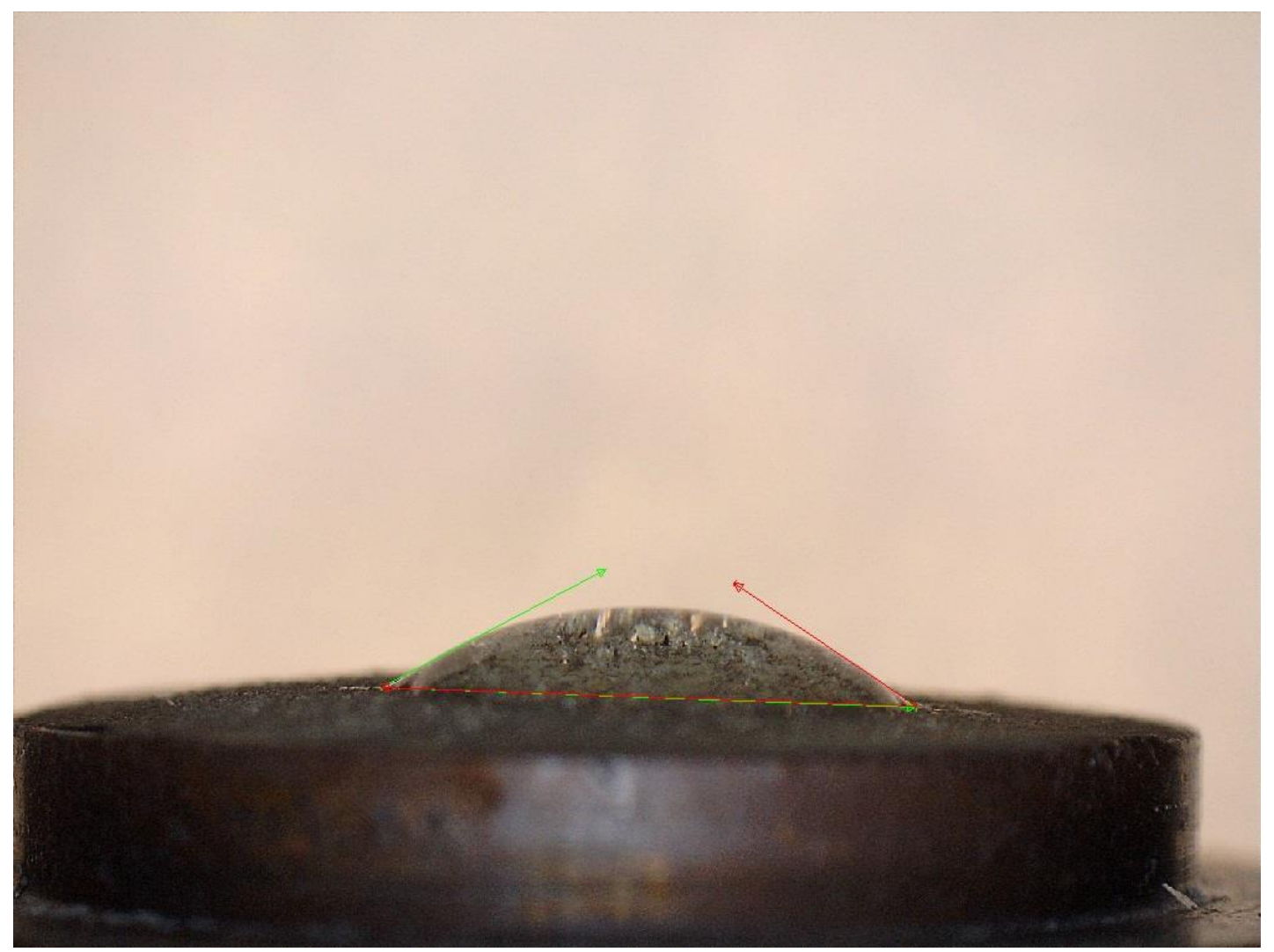

Figure 50: The same sample, after being boiled in DI water. The surface's wettability has changed, and now exhibits hydrophilicity. 


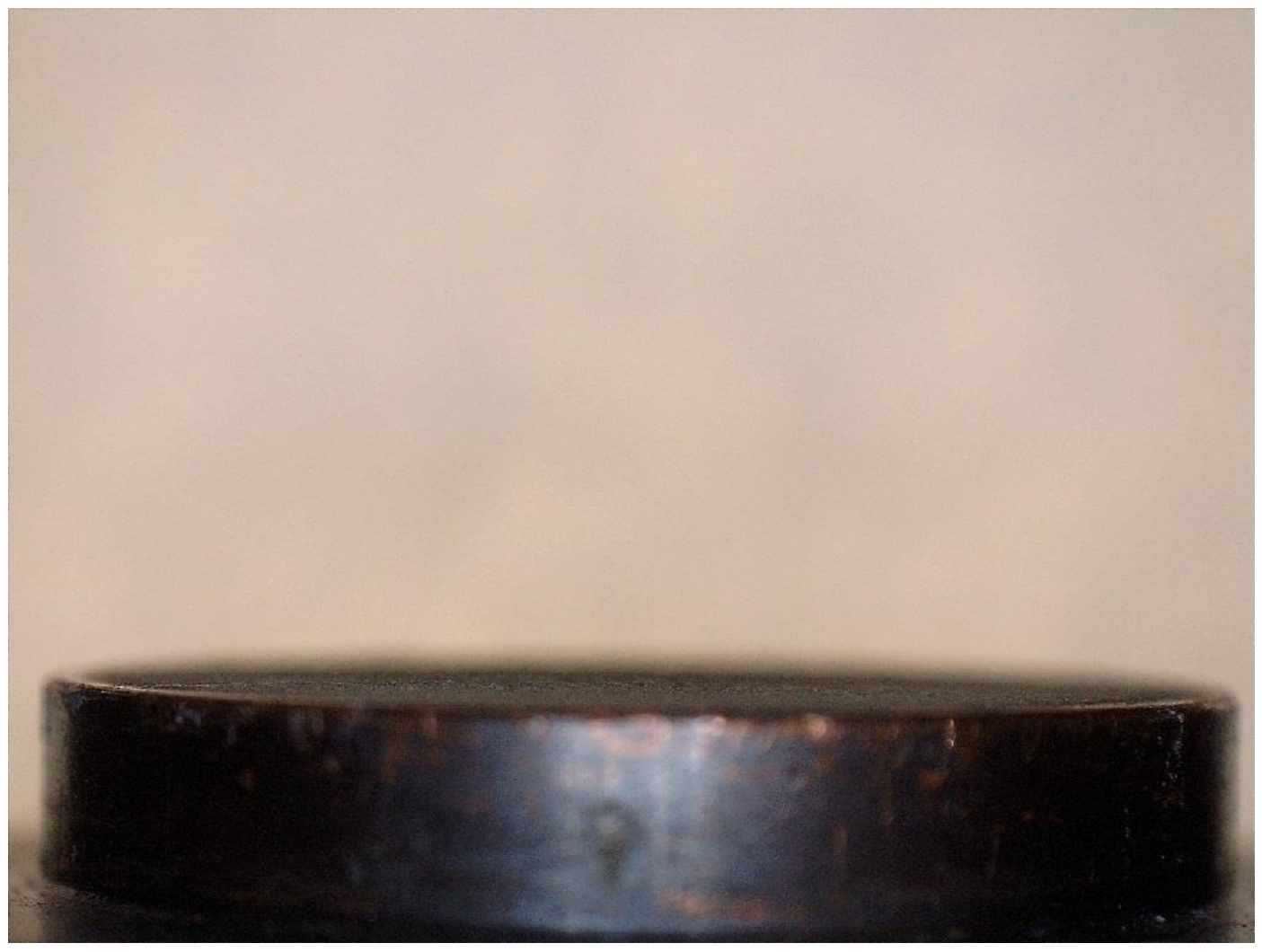

Figure 51: An example of a surface exhibiting a contact angle of $0^{\circ}$. The droplet is completely invisible from the perspective of the camera.

\subsubsection{Contact angle measurement procedure}

When measuring contact angle, a single water drop is dispensed onto the surface from a 20gauge needle, and the surface is placed in front of the camera. The focus is adjusted so that the edges of the droplet are as sharp as possible, and the internal lighting is turned on. The light box is placed over the whole system, and then a picture is taken using the software MicroCapture Pro. This is repeated five times, with the droplet being positioned in different regions of the surface to get an overall average contact angle.

These images are then imported into NI Vision Assistant, where the angle between the base of each droplet and each of its sides is measured, giving a total of ten angles. The average of these angles is used as a reasonable approximation of the contact angle. It should be noted, however, that because the camera is not perfectly aligned with the surface that this angle will be slightly wrong, so these values should only be used to loosely categorise the contact angles.

In some cases, the water droplet will not form a drop shape on the surface but will instead be absorbed into it. Surfaces that exhibit this behaviour are assigned a contact angle of $0^{\circ}$. 


\subsection{Surface analysis}

As was mentioned in section 2.7, the contact angle of FCS surfaces has been observed to change with respect to the mass loading of silver in the initial solution. In those experiments, the mass of the solution deposited was never varied, and so it is possible that the effect is due not to the concentration of solution, but to the total mass of silver deposited instead. The same is true of PVA mass deposited. When fabricating the FCS surfaces for this project, the concentrations of silver and PVA in the solution used in fabrication were varied, as was the mass deposited. The contact angle was recorded before and after the boiling process, and these contact angles were compared to each other to see if any patterns could be ascertained.

\subsubsection{Silver mass}

The total mass of silver was found by recording the mass of the solution deposited on the scale and multiplying that number by the concentration of silver in the solution. The contact angle should decrease with respect to silver mass in the post-boiling results. The results for both pre- and post-boiling are shown in Figure 52.

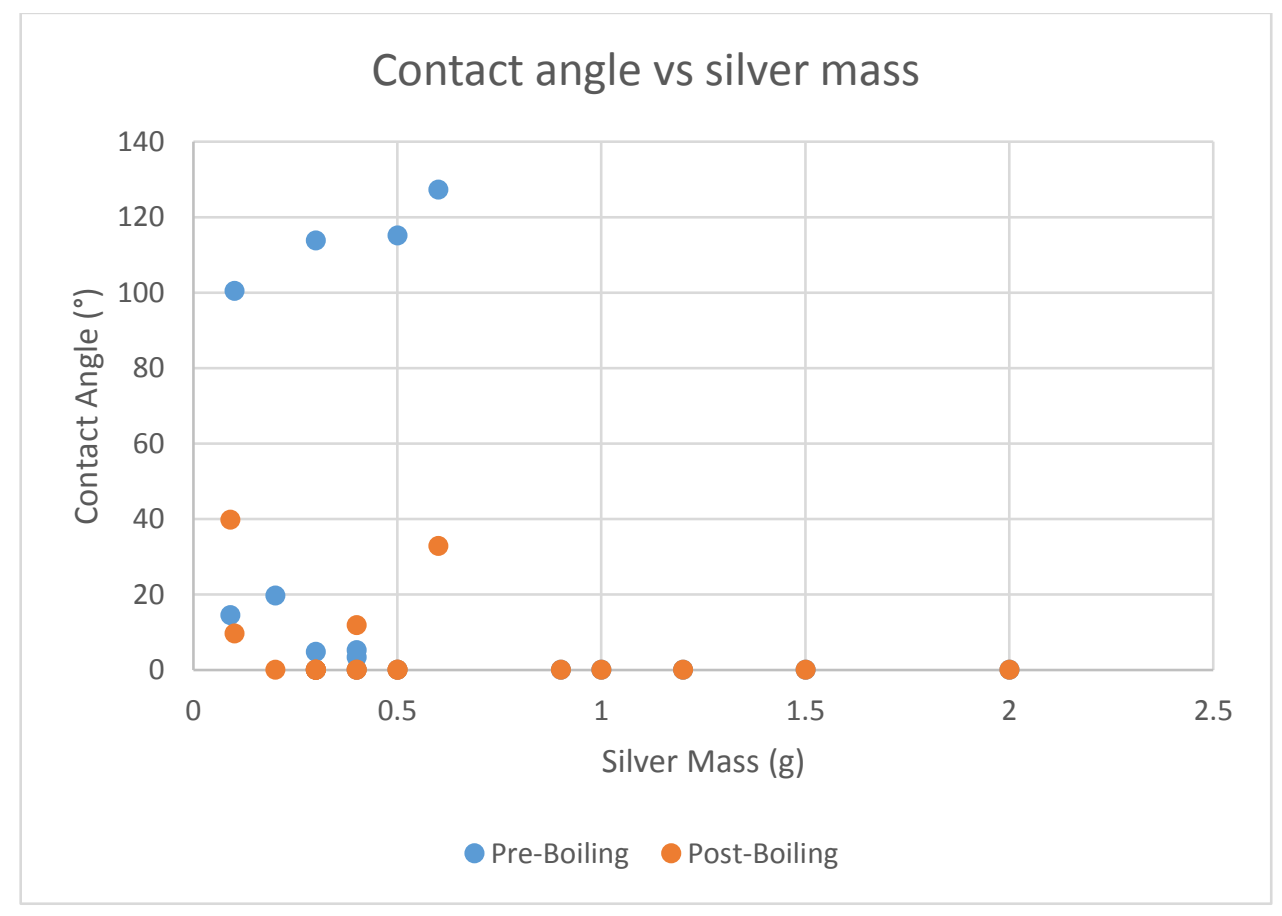

Figure 52: Contact angle vs silver mass, before and after boiling.

The post-boiling contact angle was somewhat unpredictable below $0.5 \mathrm{~g}$ of silver, but a general trend of decreasing contact angle as silver mass increased is present. More notably, 
above around $0.75 \mathrm{~g}$ of silver, the contact angle dropped sharply, with effectively $0^{\circ}$ contact angles on all surfaces both before and after being boiled. Also clear in this graph is the overwhelming difference that occurs in contact angle when comparing samples before and after being boiled.

\subsubsection{PVA concentration}

In addition to the mass of silver used in the solution, the mass of the PVA was also considered. PVA is water soluble, so it would be expected that, if any is left in the porous layer, it will increase the wettability. The results for both pre- and post-boiling are shown in Figure 53.

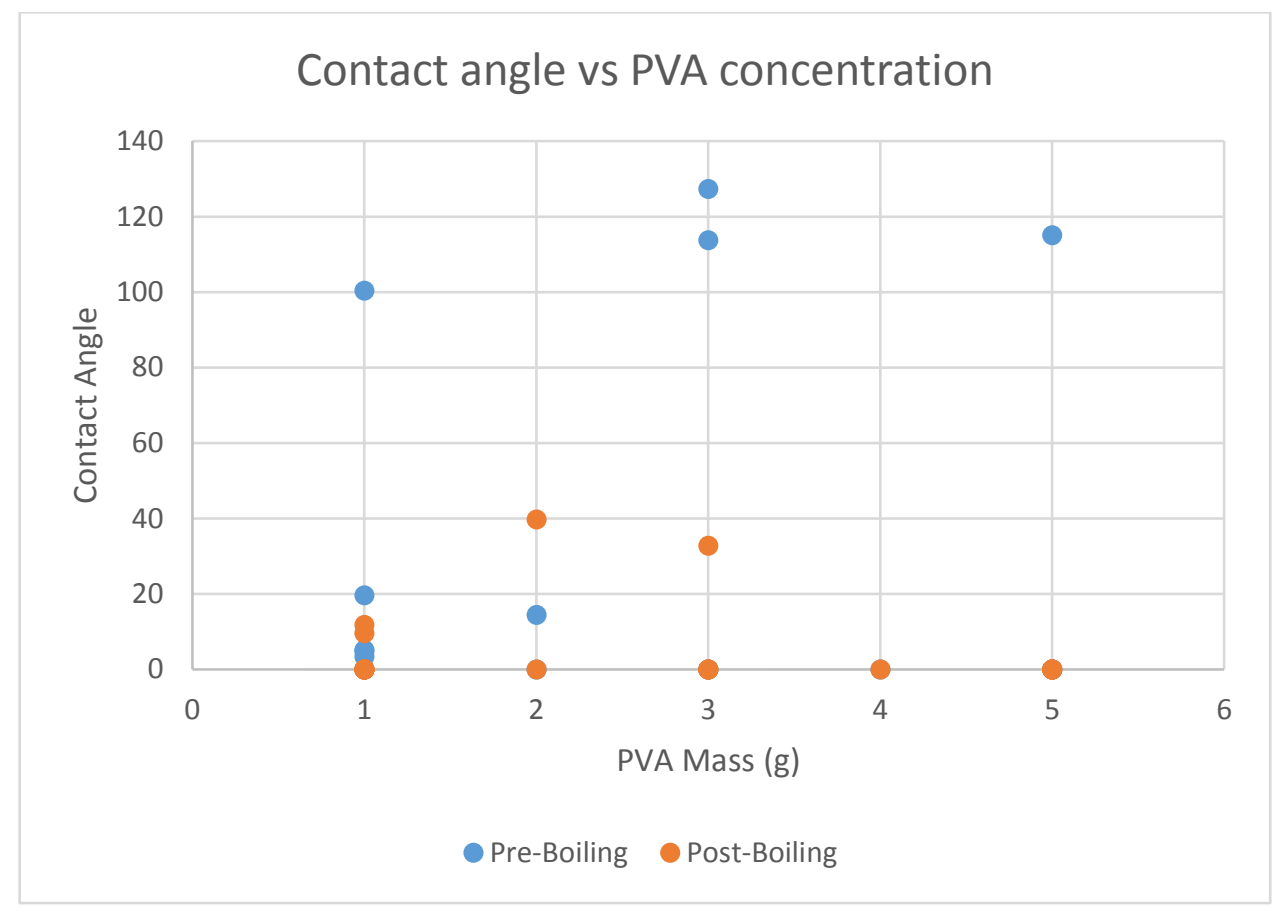

Figure 53: Contact angle vs PVA mass, before and after boiling.

The results do show that, in post-boiled surfaces, a higher PVA mass is more likely to result in a lower contact angle. However, this trend is weak, and it is difficult to conclude how significant the effect of PVA mass is on wettability.

\subsection{Fabrication of other surfaces}

In addition to the FCS surfaces, several additional surfaces were manufactured. Each of these surfaces served a different role, and these roles will be explained in this section. The methods of fabrication for each surface will also be detailed. 


\subsubsection{Lost-carbonate copper}

The purpose of the lost-carbonate copper surface was two-fold. Firstly, it provided an alternative porous surface for comparison against the FCS surfaces. Secondly, as it is very rigid in comparison to the FCS surfaces, it allowed the pressure differential capabilities of the sensing system to be established. This surface made use of a Versarien low profile metallic foam heat sink [53], a commercially made porous copper heat sink which is fabricated using the lost mass technique mentioned in section 2.5.3.1. This heat sink is designed for air cooling, but its structure should improve liquid cooling as well. The pores on this heat sink are approximately $1 \mathrm{~mm}$ in diameter on average. The heat sink was cut into circular discs the same diameter as the substrates, and then one side was cleaned so that the copper was exposed. This side was soldered on to the surface of the substrate using solder-paste to establish a good thermal contact without melting the heat sink.

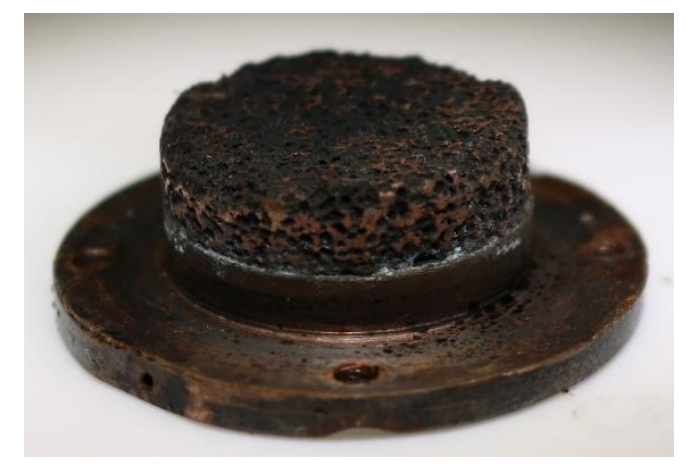

Figure 54: Versarien porous copper substrate

\subsubsection{Pin Copper}

Establishing that the test system is operating appropriately was an important component of this project. Making changes that are known to increase heat transfer and confirming that they do was one mechanism used to achieve this goal. One of these changes was fabricating a second version of the control substrate that has pins to increase the total surface area by a predictable amount. The surface was cut with $1 \mathrm{~mm}$ deep grooves whose central angle was $45^{\circ}$. These were cut horizontally and vertically across the surface, with a distance of $1 \mathrm{~mm}$ between each trough, increasing the area of the surface by a factor of around 2.3. 


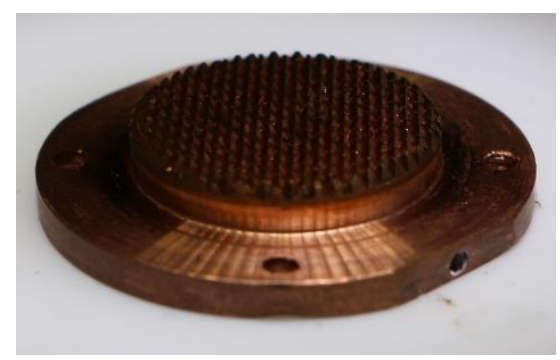

Figure 55: Pin copper substrate.

\subsubsection{Mesh Fins}

Initially, it was planned to produce an FCS surface that filled the chamber completely. Unfortunately, the surface contracts slightly when it goes through the heating process, and so the attempts made to fabricate such a surface almost always ended in the surface destroying itself due to shear forces while in the furnace. In an attempt to overcome this obstacle, copper fins of 100 mesh were placed into one of the substrates perpendicular to the surface, to act as a framework on which to attach the FCS. These fins were soldered into slots cut into the substrate and were cut to the height of the chamber so that they barely touched the roof. After characterising the behaviour of this substrate as a control, an FCS was fabricated over it, coating the mesh fins completely in porous silver. This FCS succeeded in filling the chamber as desired.

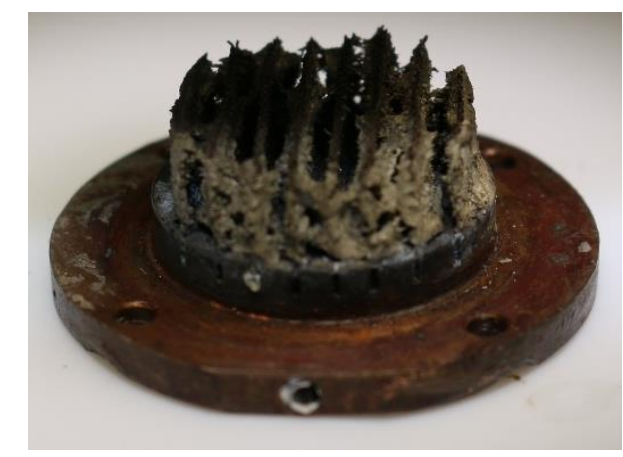

Figure 56: 100 Mesh fins substrate coated in porous silver. The mesh fins are still distinguishable in the image. 


\section{Heat transfer results}

In this chapter, results on the test system performance and heat transfer during forced liquid convection are presented. For the purposes of clarity, in this chapter the term surface will be used to describe structures that were thin enough to not cover the inlet and outlet holes of the enclosure, while substrates with structures that are thick enough to do so will be referred to as thick structure substrates. All experiments undertaken during this chapter follow the test procedure outlined in section 3.4 unless otherwise specified.

Assessment of the test system's performance is detailed in section 5.1. Details pertaining to the performance of the porous surfaces are provided in section 5.2. This section ends in a comparison of the best results from each type of surface for context. The thick structure substrate tests are compared in a similar fashion in section 5.3.

\subsection{Evaluation of test system}

The main aim of the project was to design the cooling system with integrated testing. As such, the most important results are the ones focused on the performance of the test system itself, without porous surfaces involved. These results include the performance evaluation of two thermal interface materials, thermal imaging of the system to identify areas of power loss, the performance of a commercial system to provide a sense of scale, and three "sanity checks", where the change as a result of an altered parameter with an expected response is compared to the actual response of the system, to confirm that it is acting as it should.

\subsubsection{Thermal interface material}

It is important that there is adequate thermal contact between the heat source and the substrate. To this end, a thermal interface material was used to join the two, as mentioned in section 3.1.2. Two heat transfer compounds were considered, Electrolube HTS Silicone heat transfer compound [54] and Arctic Silver 5 high density polysynthetic silver thermal compound [55]. Preferably, the thermal interface material would have a low thermal resistance and, more importantly, a low variance in its thermal resistance. To test this, a copper control substrate was put into the chamber, the heater voltage was set to $80 \mathrm{~V}$ (which correlates to an input power of $112.24 \mathrm{~W}$ ), and the heat flux the system was recorded. Then 
the pump was turned off, the surface removed, and the interface layer cleaned off and reapplied, before turning back on and recording again, five times in total. This was repeated for both interface materials. The thermal resistances of the two sets of tests are shown in Figure 57.

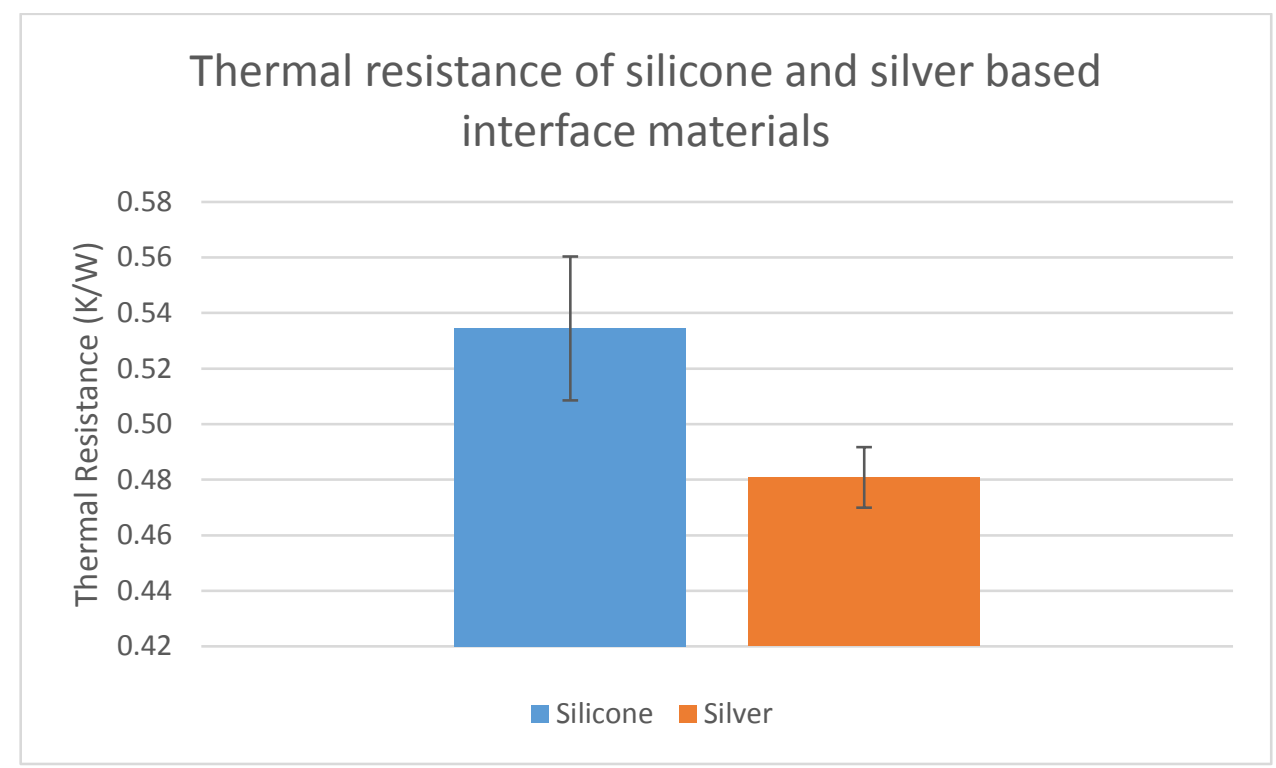

Figure 57: Thermal interface materials thermal resistance comparison.

The thermal resistance associated with the silver compound $(0.48 \mathrm{~K} / \mathrm{W})$ is significantly less than the thermal resistance associated with the silicone compound $(0.53 \mathrm{~K} / \mathrm{W})$, and the variance of the silver compound's resistance $(0.01 \mathrm{~K} / \mathrm{W})$ is much smaller than the variance of the silicone compound's resistance $(0.03 \mathrm{~K} / \mathrm{W})$. Clearly, the silver-based interface material is superior for use in this application.

\subsubsection{Flow rate}

In section 2.2.2 it was established that the convective heat transfer coefficient increases with an increase in flow velocity. As thermal resistance is inversely proportional to the convective heat transfer coefficient, and flow rate is proportional to flow velocity, this indicates that the thermal resistance should decrease with an increase in flow rate. However, the specifics of this relationship are difficult to calculate due to the nonlinear relationship between the flow velocity and the convective heat transfer coefficient. 
Four different flow rates were used to test this expectation, dispersed evenly between $1 \mathrm{l} / \mathrm{min}$ and $1.8 \mathrm{l} / \mathrm{min}$. This maximum flow rate was chosen because the pressure exerted on the walls of the pipes at flow rates higher than this value was sufficient to expand those walls at the connection points of the chamber, allowing them to pop off the connectors. Introducing pipes with rigid walls should allow for a wider range of flow rates to be tested. The thermal resistance response is shown in Figure 58.

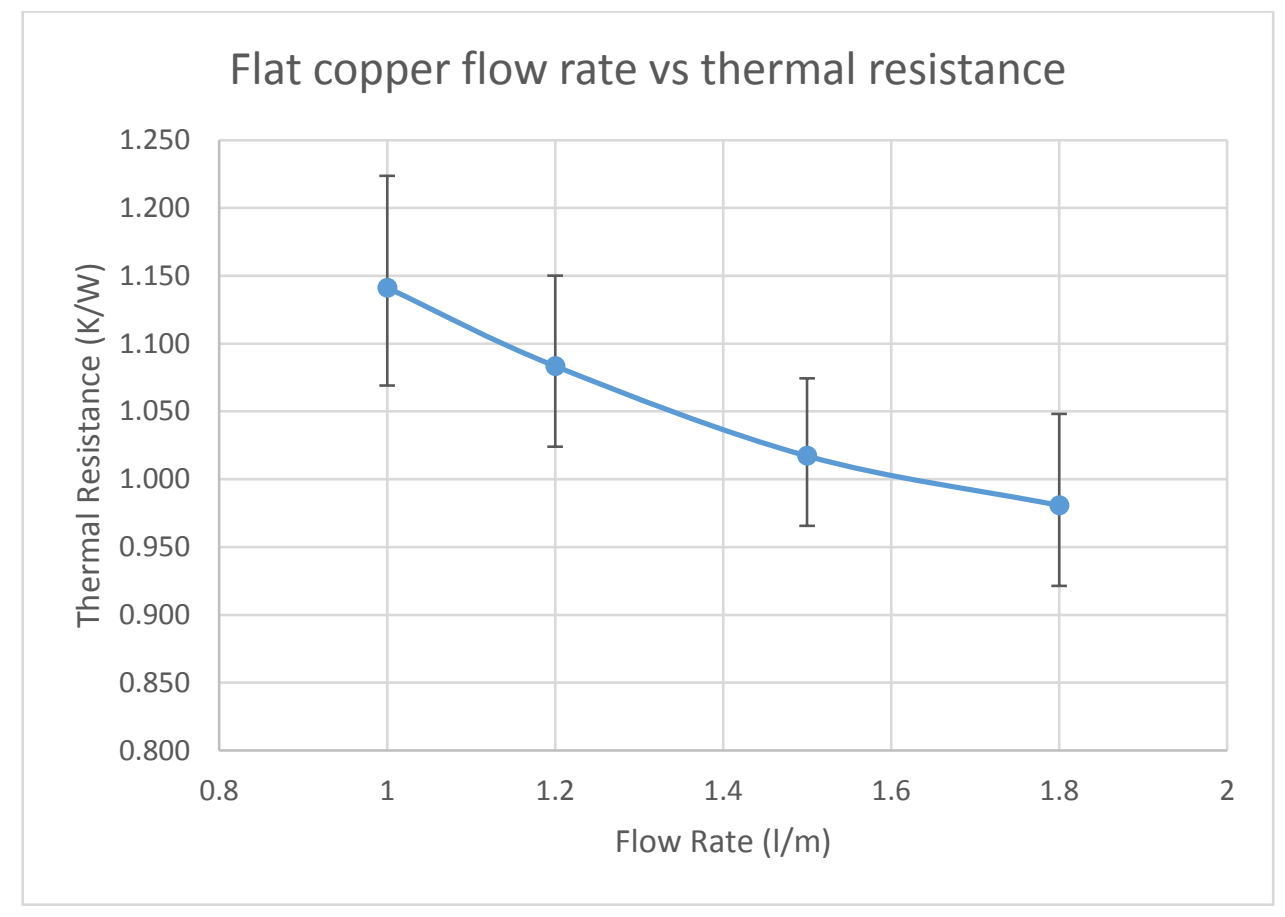

Figure 58: Flow rate vs thermal resistance for copper control.

Figure 58 clearly shows that an increase in flow rate results in a decrease in thermal resistance as expected.

\subsubsection{Flow direction}

While none of the relevant theory states that a change in the direction of flow should result in a change in the thermal resistance of the system, a slight decrease in thermal resistance was expected when the inwards flow was downwards rather than across the surface. This is due to the motion encouraging the fluid in direct contact with the surface to move more effectively than the horizontal inlet, which is a few millimetres above the surface and could result in a small pocket of liquid beneath the flow essentially operating under free convection principals. 
To test this, the vertical flow roof was connected to the enclosure, and the flow rate tests were repeated using this setup. The flexibility of the silicone tubing was useful here, as the connection to a completely different area on the enclosure did not require significant alterations to the piping beyond the inclusion of a T-connector on the outlet end of the system to take flow from both the outlet and built-in inlet. The thermal resistance response for these tests is shown in Figure 59 alongside the results from Figure 58.

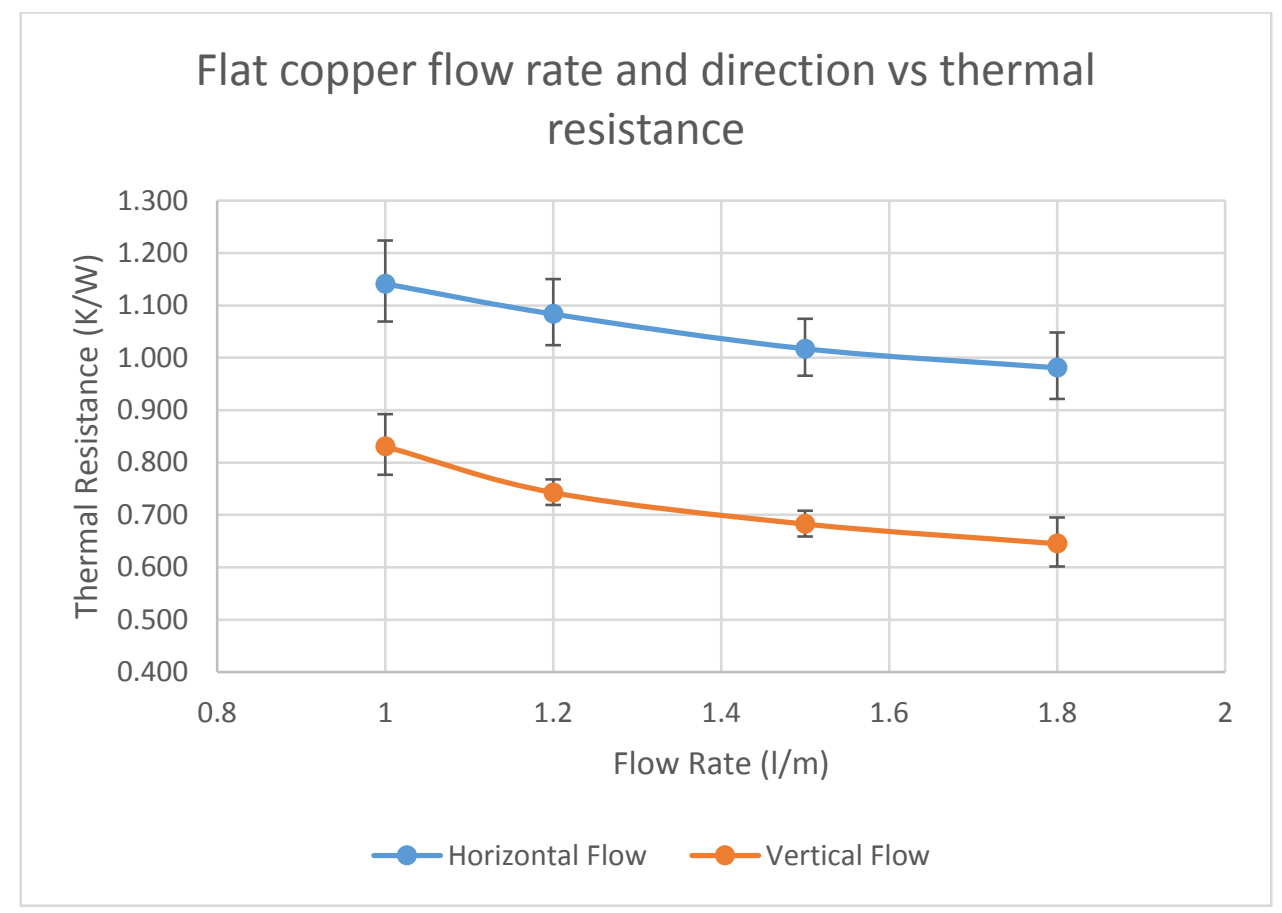

Figure 59: Flow rate and direction vs thermal resistance for copper control.

Figure 59 shows that, as expected, the vertical flow rate decreased the thermal resistance significantly. Overall, the thermal resistance of the control under vertical flow was about 1.5 times smaller than that of the control under horizontal flow.

\subsubsection{Pin copper}

As was discussed in section 2.3, the convective thermal resistance is inversely proportional to the area of the surface in thermal contact with the coolant. Furthermore, the conductive thermal resistance over a solid is proportional to the distance from the heat source to the surface. It follows that cutting into the surface of a substrate that is otherwise identical to the copper control (such that the surface area is increased and the distance from the heat source is decreased) should reduce the thermal resistance significantly. 
This was tested using the pin copper surface discussed in section 4.4.2. This surface had an area of approximately 2.3 times more than the copper control, with an average distance to the surface that was approximately $0.4 \mathrm{~mm}$ less. Assuming that the conductive thermal resistance of the substrate and the convective thermal resistance between the substrate and the fluid are the only two thermal resistances, this predicts a decrease in thermal resistance by a factor of $\sim 2.5$. This gives an upper bound to this check. The thermal resistance should decrease but decreasing by more than a factor of 2.5 means the system is not correctly measuring the thermal resistance. The flow was tested vertically using the same four flow rates to ensure that the fluid would penetrate the pins. The thermal resistance response for these tests is shown in Figure 60 alongside the vertical flow results from Figure 59.

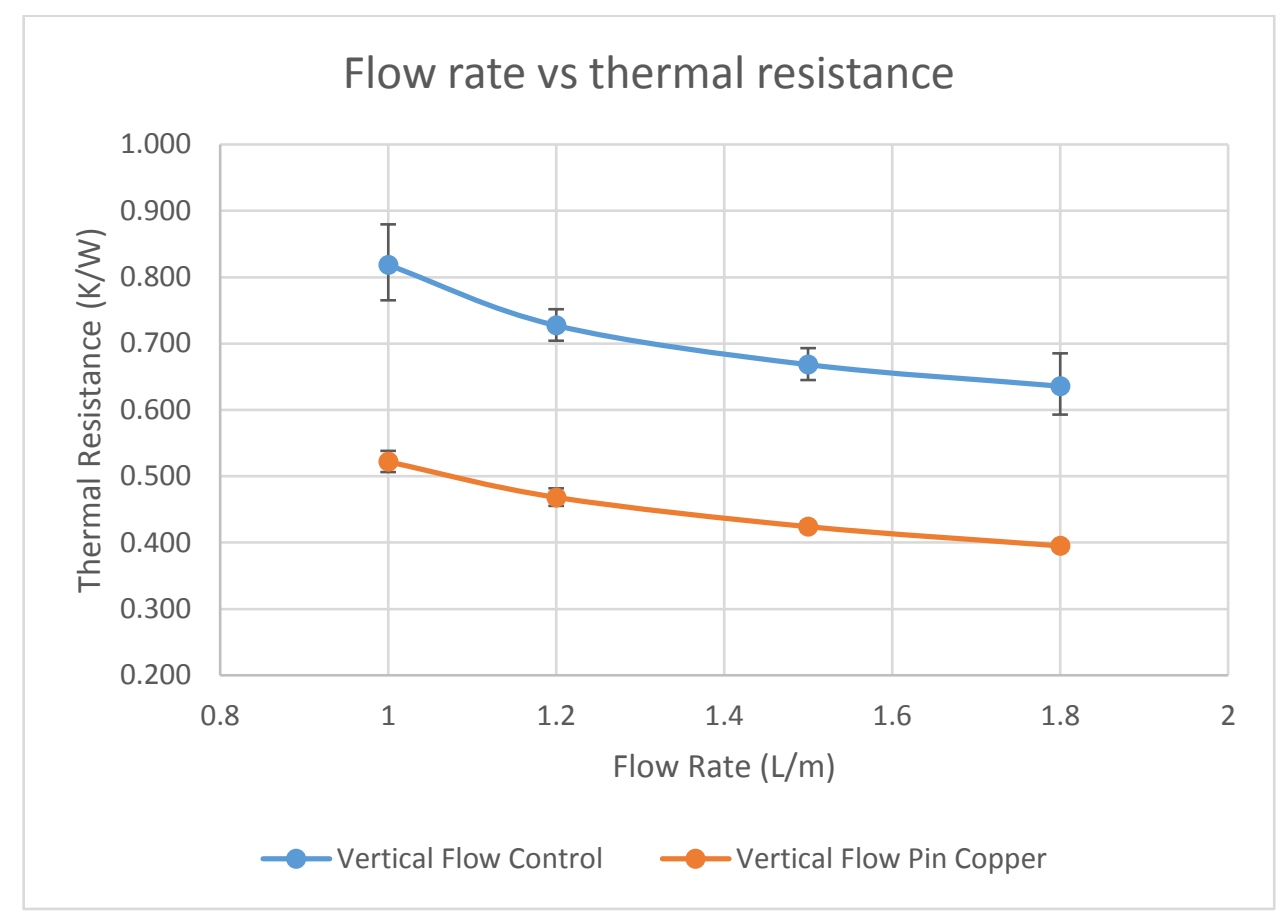

Figure 60: Flow rate vs thermal resistance for copper control and pin copper.

As is shown in Figure 60, the pin copper performed significantly better than the copper control. However, it also performed significantly less well than the calculated maximum. This is good, as the assumption that the only thermal resistances in the system were the conduction and convection through and from the substrate is clearly false. The thermal resistance associated with the heat source is obviously being excluded, as are the thermal resistances associated with each other element in the system. The pin copper reduced the copper control's thermal resistance by a factor of approximately 1.6. 


\subsubsection{Analysis of heat loss in system}

One detail not clear in the previous graphs, but clear from the results as a whole, is that the heat fluxes being calculated were significantly lower than expected, with less than half the input power actually flowing through the surface to be dissipated by the coolant. The obvious conclusion of this discovery is that power must be being lost elsewhere in the system.

Due to this high power loss, infrared pictures were taken of the system both before and during operation using a FLIR T440 infrared camera [56]. As the metallic outer casing of the insulation ring is reflective in the infrared spectrum, a piece of tape was attached to one side. This would reach a similar temperature to the wall and is not reflective, allowing the surface temperature to be visible. A thermocouple was also directly attached to the side to measure the temperature.

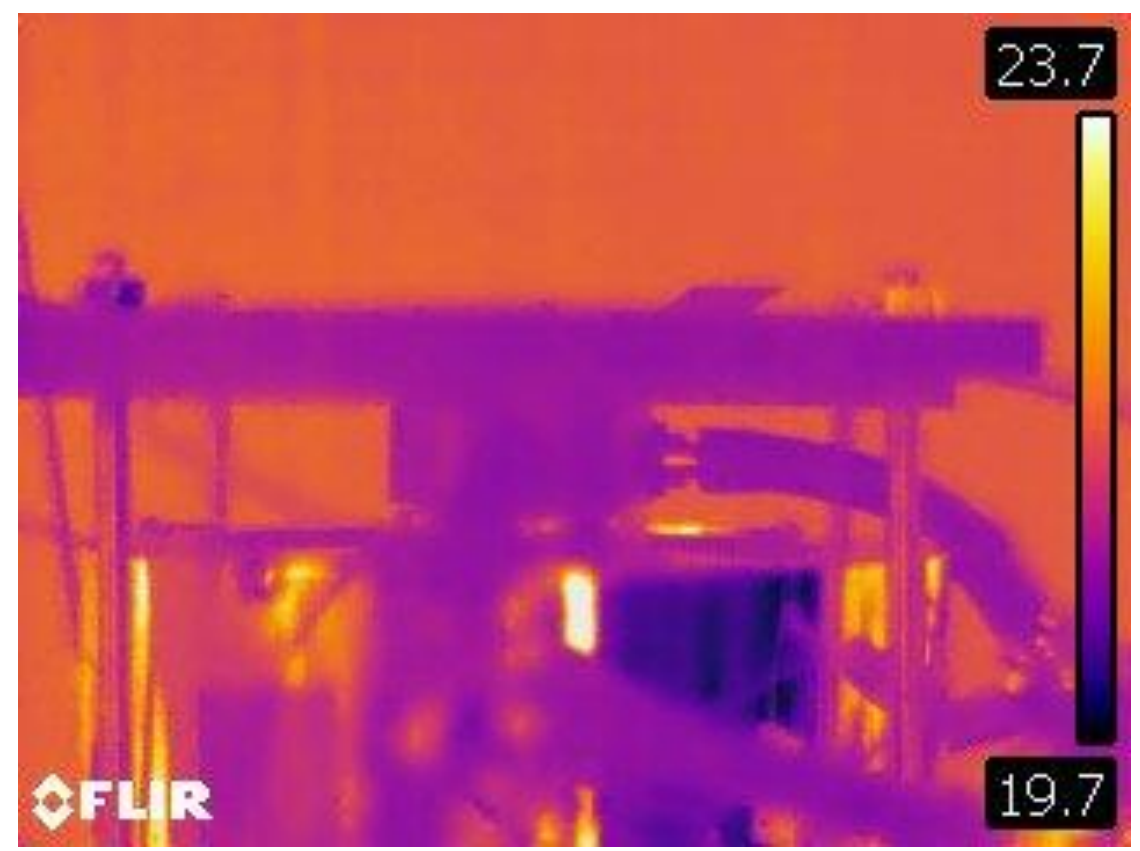

Figure 61: Infrared picture of surface and wall with system off. 


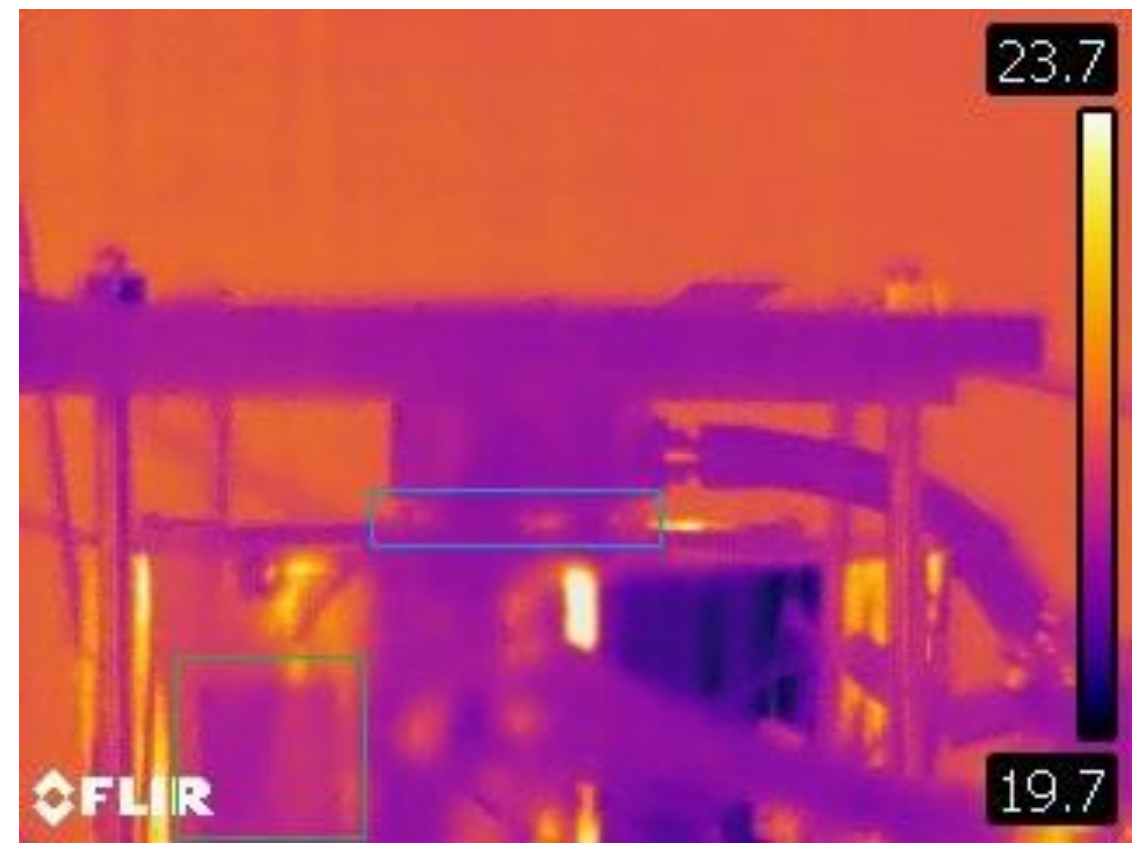

Figure 62: Annotated version of Figure 61. The green box shows the tape that reduces infrared reflection, and the blue box shows the thermal interface between the surface and the substrate.

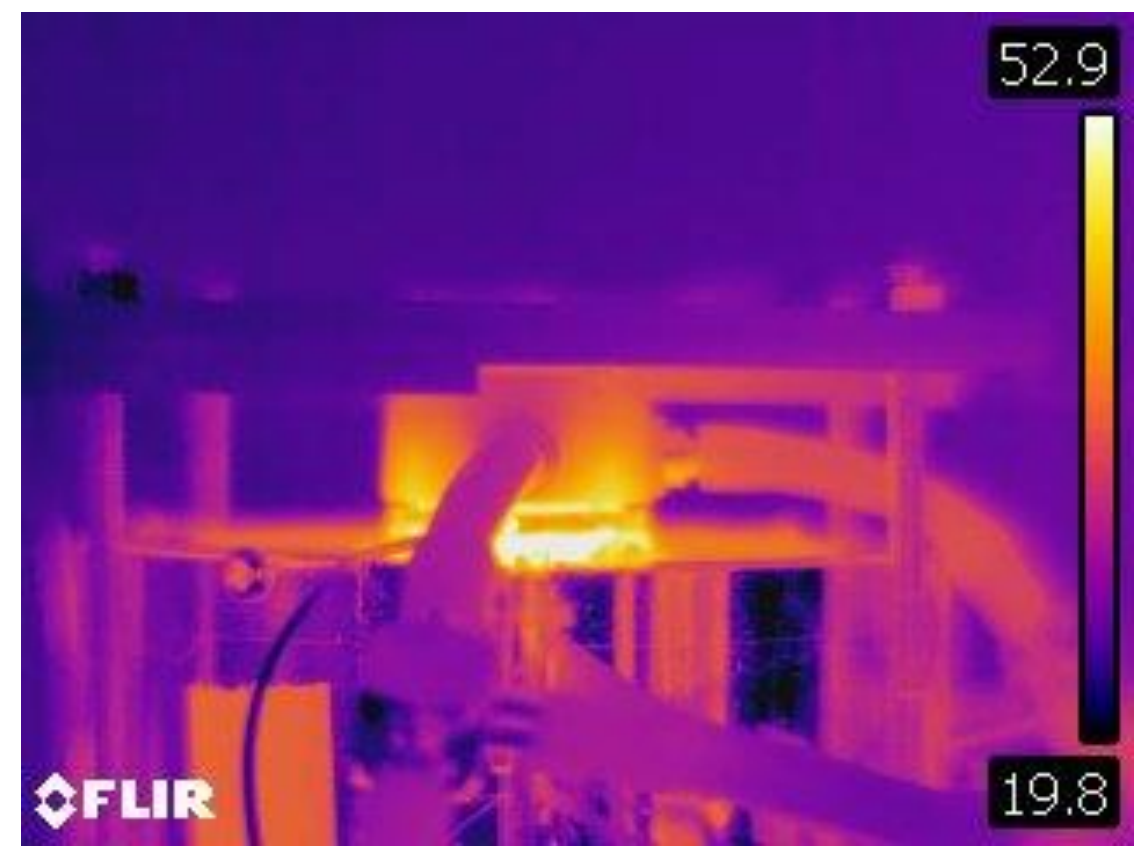

Figure 63: Infrared picture of surface and wall with system on, showing thermal leakage.

The tape shows that the temperate at the wall of the insulation ring is approximately $30^{\circ} \mathrm{C}$, which agrees with the $28.8^{\circ} \mathrm{C}$ measured using the thermocouple. The temperature of the surface registers the maximum recorded temperature $\left(52.9^{\circ} \mathrm{C}\right)$, which compares well with the internal temperature measured at $60.3^{\circ} \mathrm{C}$. Both of these are likely causing significant power 
loss, due to the high temperature of the exposed heating column, and the high surface area of the insulation ring.

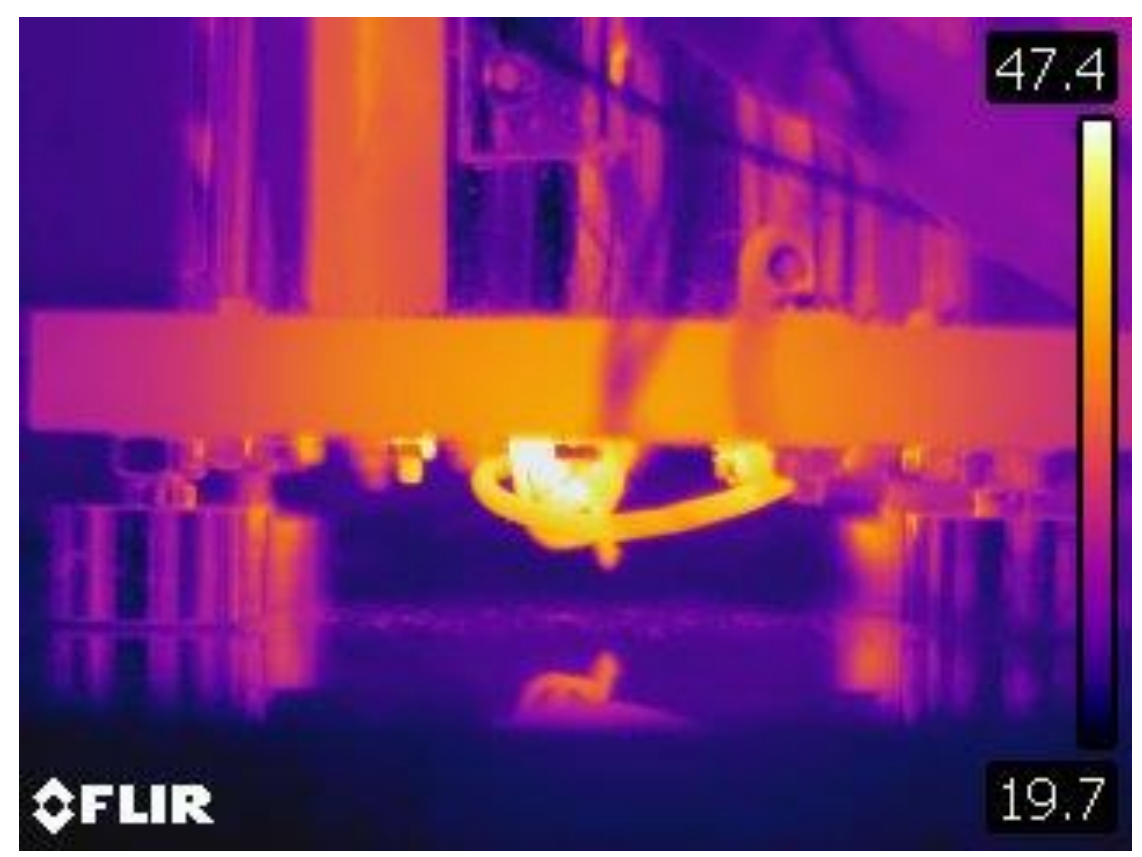

Figure 64: Infrared picture of base of heater column with system on, showing thermal leakage.

The internal temperature at the tip of the heaters registered at $70.5^{\circ} \mathrm{C}$, which is much higher than the infrared recorded temperature of $47.4^{\circ} \mathrm{C}$, but the former temperature is measured both half-way up the column and in the centre of it. There is also the ceramic base separating the heater column from the area visible. It is entirely possible that this heat is coming from the resistance of the wires feeding into the heaters, not the heaters themselves, though this is still a power loss from the electrical input.

These results show there are some major thermal leaks, which explains why less than half the power is being transferred into the fluid. Clearly, there is room for improvement with regards to insulating the system.

\subsubsection{Test of a Corsair H110 commercial liquid cooling system}

To get a sense of scale for the thermal resistances being analysed, a Corsair H110 liquid cooling system [57] was attached to the heat flux meter and its thermal resistance was measured. This system is designed to be used for cooling the CPU or GPU of a high- 
performance PC, and so it should have a significantly lower thermal resistance to the copper control as long as the pump is running at a similar capacity.

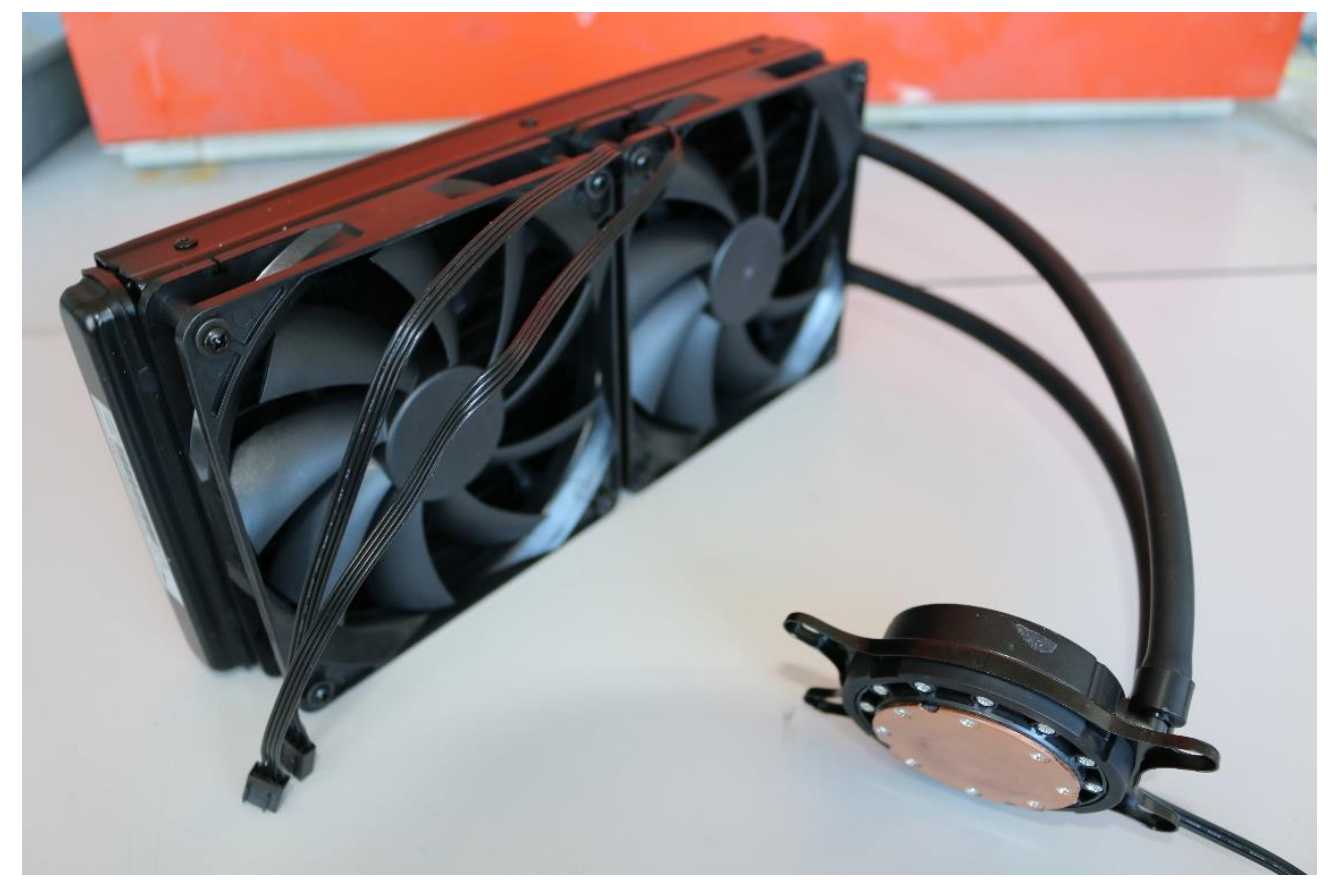

Figure 65: Corsair H110 liquid cooling system.

Because the commercial system needed the heat exchanger fans to be powered as well, ensuring the same pumping power was provided was not feasible. However, as voltage does not change at parallel junctions, the two pumps could be run at the same input voltage. This resulted in a flow rate of $\sim 1.5 \mathrm{l} / \mathrm{min}$ for the test system. Figure 66 shows the thermal resistances of each system for comparison, with both the copper control and pin copper substrates included. As the pin copper used vertical flow, the copper control thermal resistance is also under vertical flow. 


\section{Thermal resistances of different surfaces: copper}

control vs commercial system

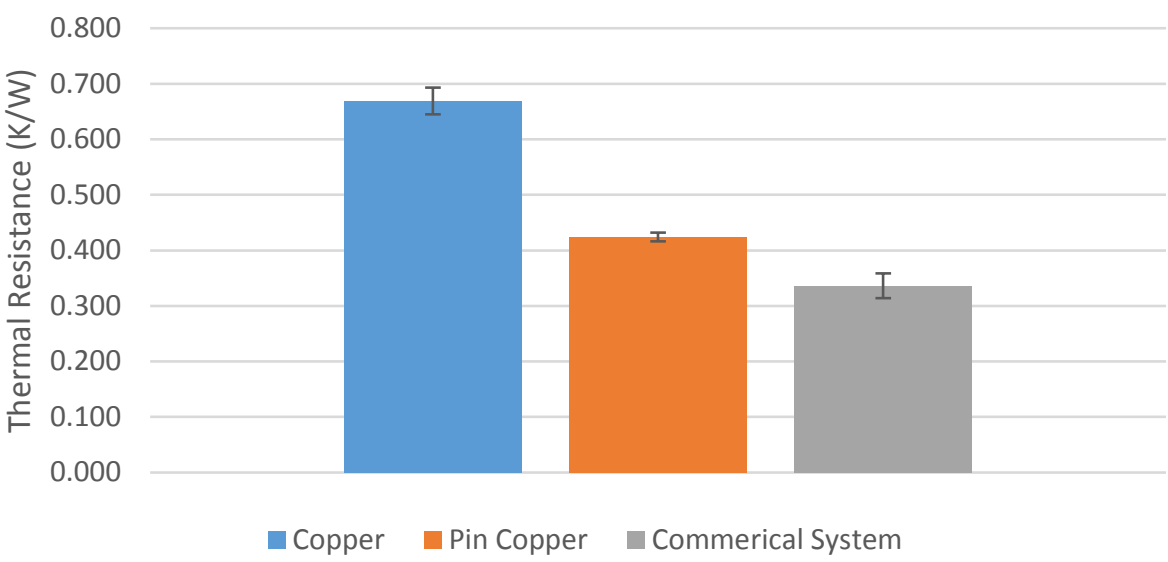

Figure 66: Copper control in test system vs Corsair H110 commercial system.

As is clear from Figure 66, the commercial system performed significantly better than the copper control, as it only had half the thermal resistance. Pin copper performed relatively close to the commercial system, with a thermal resistance only 1.25 times higher. It should be noted that statistics like the coolant liquid, the coolant flow rate, or the surface area of the cold plate in contact with the coolant are unknown in this case.

\subsubsection{Test system summary table}

\begin{tabular}{|c|c|c|c|c|}
\hline $\begin{array}{c}\text { Substrate } \\
\text { Details }\end{array}$ & Test setup & Media description & $\begin{array}{c}\text { Coolant flow } \\
\text { rate (I/min) }\end{array}$ & $\begin{array}{c}\text { Thermal } \\
\text { Resistance (K/W) }\end{array}$ \\
\hline Copper Control & Horizontal flow & Flat copper & 1 & 1.141 \\
\hline Copper Control & Horizontal flow & Flat copper & 1.8 & 0.964 \\
\hline Copper Control & Vertical flow & Flat copper & 1 & 0.818 \\
\hline Copper Control & Vertical flow & Flat copper & 1.8 & 0.636 \\
\hline Pin Copper & Vertical flow & Roughened copper & 1 & 0.468 \\
\hline Pin Copper & Vertical flow & Roughened copper & 1.8 & 0.395 \\
\hline $\begin{array}{c}\text { Commercial } \\
\text { System }\end{array}$ & N/A & $\begin{array}{c}\text { Regular } \\
\text { microchannels }\end{array}$ & N/A & 0.335 \\
\hline
\end{tabular}

Table 7: Summary table of key results from the test system evaluation. 


\subsection{Evaluation of porous surfaces}

Evaluating the performance of FCS surfaces was the secondary focus of this project. This required using the established system to measure the thermal resistance of FCS surfaces in the place of the substrate. In addition, the lost-carbonate copper surface mentioned in section 4.4.1 was measured to provide some scale for comparison.

\subsubsection{Contact angle of silver surfaces}

As discussed in section 4.1.2, an increase in surface wettability should result in a corresponding decrease in the convective heat transfer coefficient. As such, a lower wettability should result in a lower thermal resistance.

For these tests, a number of different FCS surfaces were fabricated. Unfortunately, the vast majority of them had a contact angle of zero degrees. Only four surfaces resulted in a nonzero contact angle, and two of these surfaces became unusable due to deterioration as mentioned in section 2.7. This meant that only three unique contact angles were available for the tests. These three surfaces were tested in both horizontal and vertical flow, and with both $1 \mathrm{l} / \mathrm{min}$ and $1.8 \mathrm{l} / \mathrm{min}$ flow rates. The horizontal flow results are shown in Figure 67 , and the vertical flow results are shown in Figure 68.

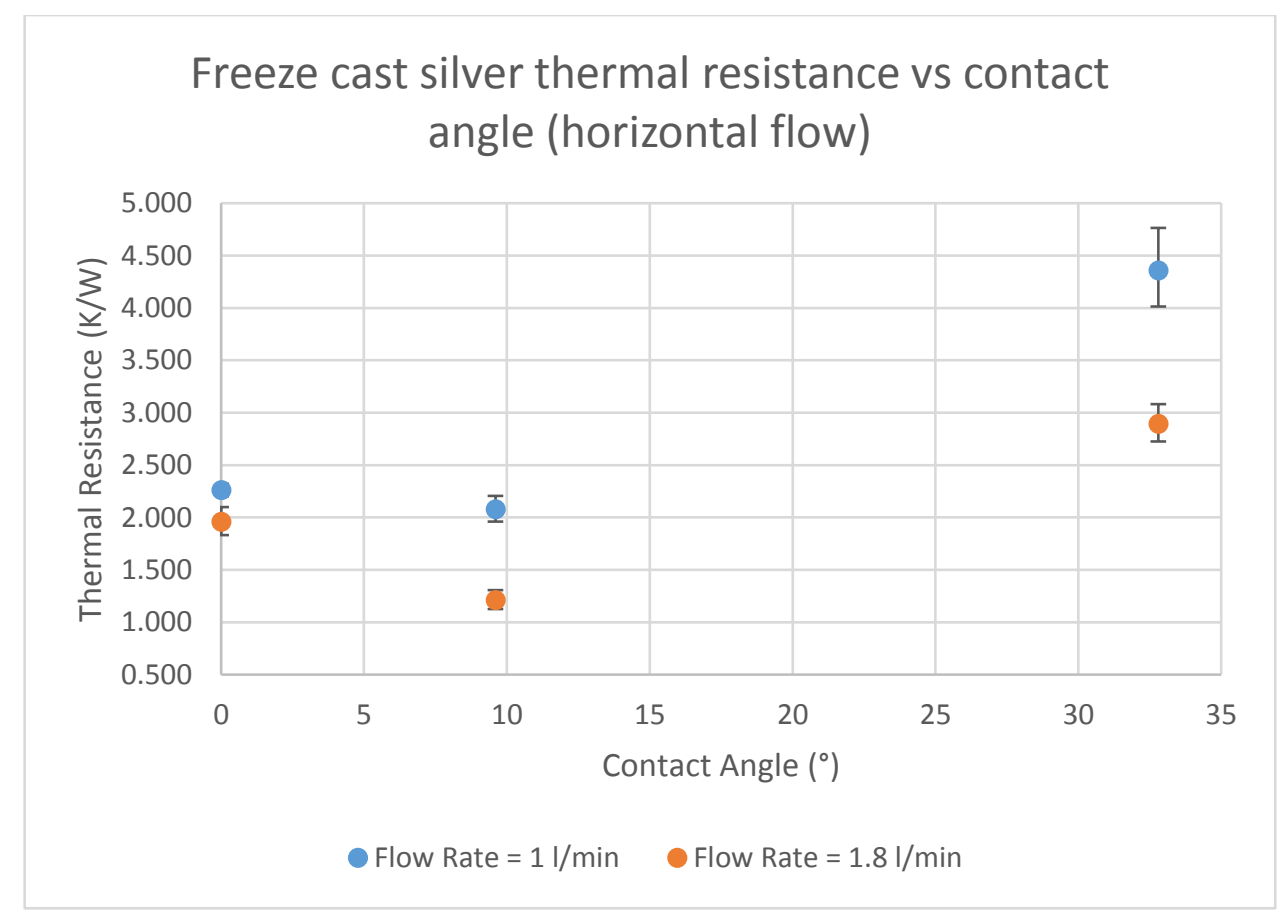

Figure 67: Thermal resistance vs contact angle at both $1 \mathrm{l} / \mathrm{min}$ and $1.8 \mathrm{l} / \mathrm{min}$ horizontal flow. 


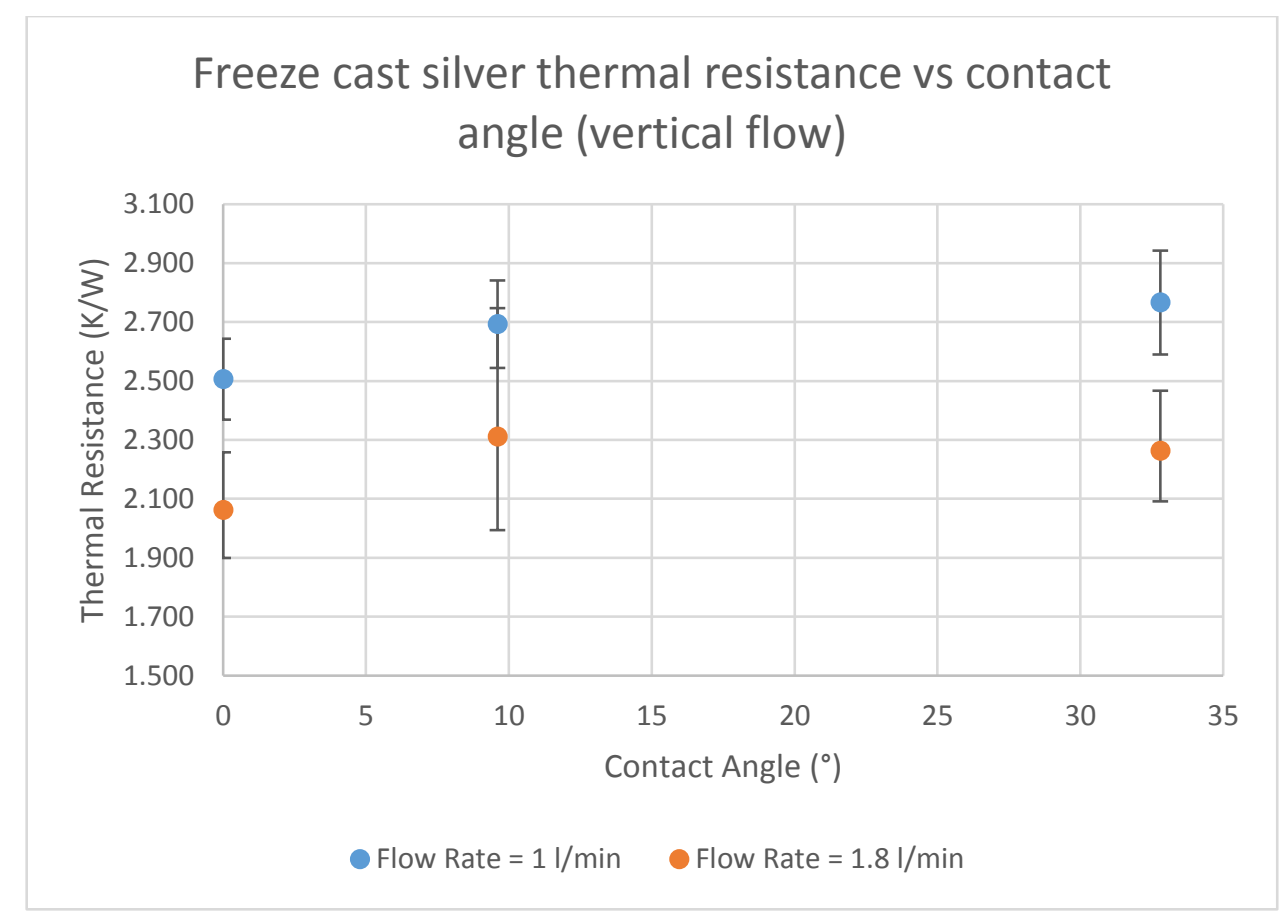

Figure 68: Thermal resistance vs contact angle at both $1 \mathrm{l} / \mathrm{min}$ and $1.8 \mathrm{l} / \mathrm{min}$ vertical flow.

Due to the low sample size, it is difficult to conclude if there is any relationship between contact angle and thermal resistance in FCS surfaces. However, it is clear that if such a relationship exists, it is not monotonic. Flow direction also appears to affect the surfaces differently, particularly the surface with a contact angle of $9.6^{\circ}$, as this moves from being the surface with the lowest thermal resistance under horizontal flow to tie for the highest thermal resistance under vertical flow. Both these results imply that contact angle is not the only statistic regarding heat transfer that varies between these surfaces.

On a more positive note, a change in flow rate does as it should, reducing thermal resistance for each surface and maintaining the curve shape under each flow direction.

\subsubsection{Performance of commercial porous copper material}

The porous copper appears to be mostly closed pores, which means only the surface has any effect on the convective heat transfer coefficient. With this in mind, the thickness of the porous copper should have an inverse relationship with the convective heat transfer coefficient, and so a thicker layer of porous copper should result in a higher thermal resistance. 
To test this, a porous copper puck was cut to $2.5 \mathrm{~mm}$ thick and attached to a substrate as outlined in section 4.4.1. After each set of tests, the copper puck was milled another $0.5 \mathrm{~mm}$ thinner, reheated to re-oxidize the surface, and tested again. This was done until the surface was $1 \mathrm{~mm}$ thick, as the $0.5 \mathrm{~mm}$ thick surface did not appear to have much in the way of porous structures. The thermal resistance response of these surfaces for both horizontal and vertical flow at $1 \mathrm{l} / \mathrm{min}$ is shown in Figure 69, with the copper control shown as a $0 \mathrm{~mm}$ layer of porous copper.

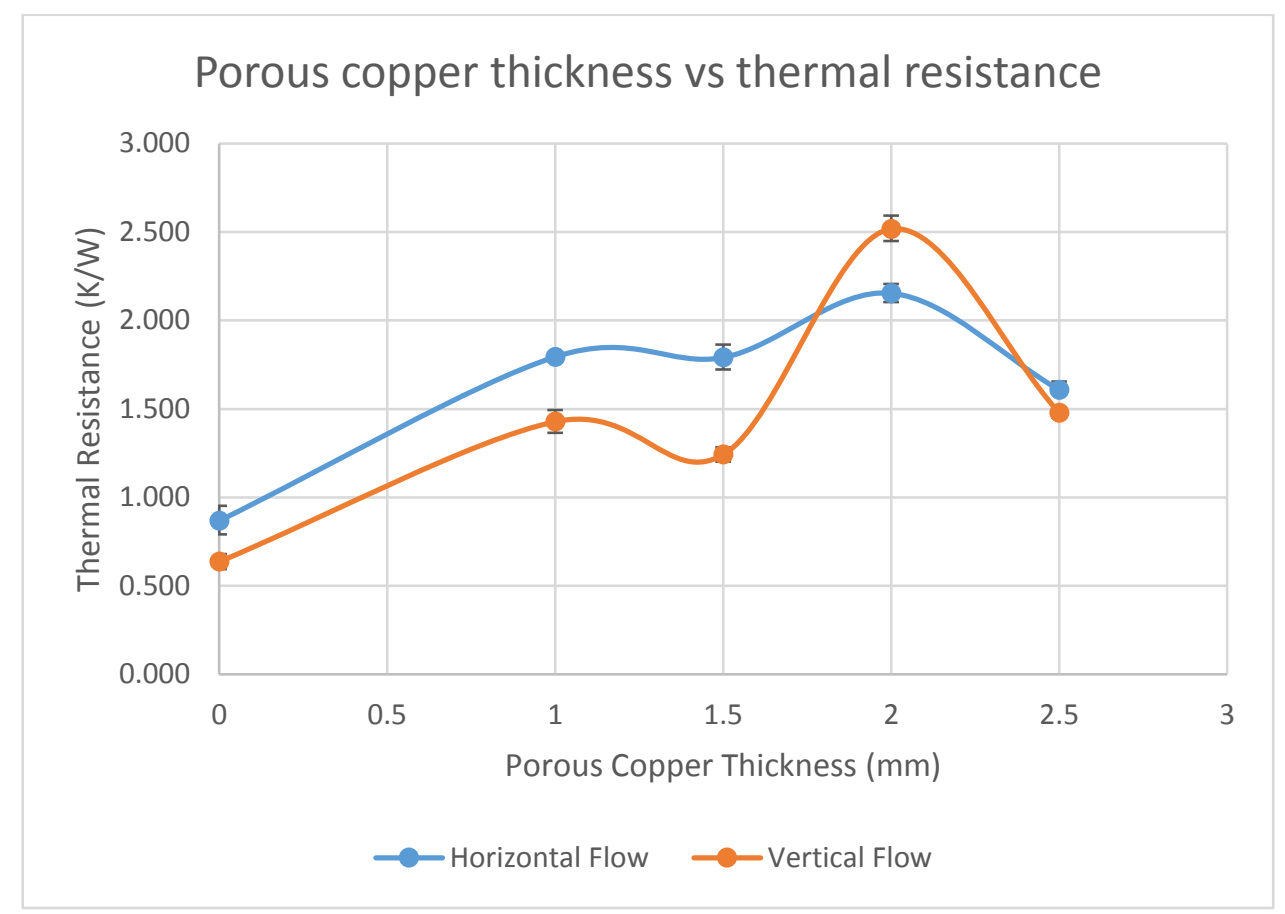

Figure 69: Porous copper thickness vs thermal resistance. $0 \mathrm{~mm}$ porous copper is the copper control.

The results, shown in Figure 69, are confusing to say the least. The shape is consistent between the horizontal and vertical flow tests, but little else appears to make sense. There is no discernible pattern explaining how the thickness of the copper correlates to the thermal resistance. However, as the performance of the porous copper was not a goal of the project, this was not investigated further.

\subsubsection{Best results}

As mentioned in sections 2.3 and 2.5 , the purpose of using porous media as surfaces is to increase the surface area and decrease the characteristic length, as both of these changes predict a decrease in thermal resistance. 
As such, the lowest thermal resistance of each of the porous mediums was compared with the results of the copper control at the same flow rate and direction. Both horizontal and vertical flow at $1 \mathrm{l} / \mathrm{min}$ were compared this way, are the comparisons are shown in Figure 70 and Figure 71.

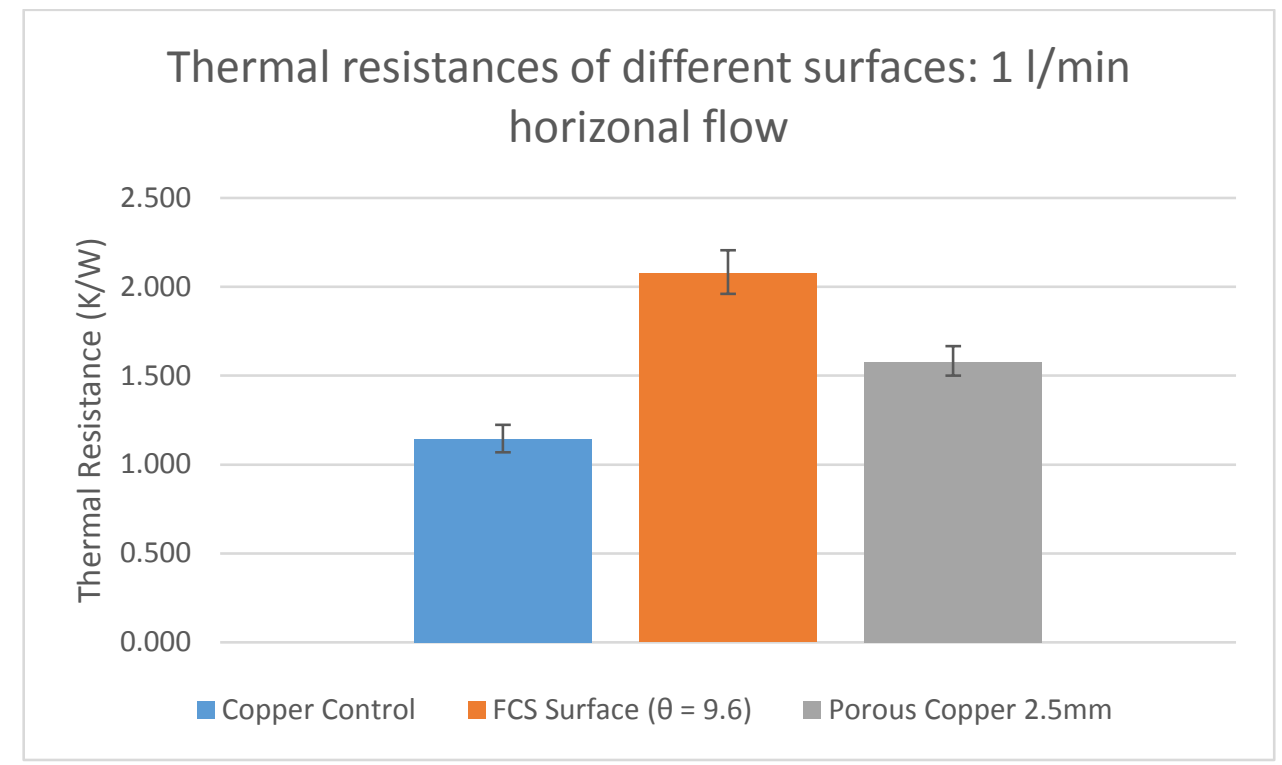

Figure 70: Comparison of thermal resistances under $1 \mathrm{l} / \mathrm{min}$ horizontal flow. Only the lowest thermal resistance of each type of surface is shown.

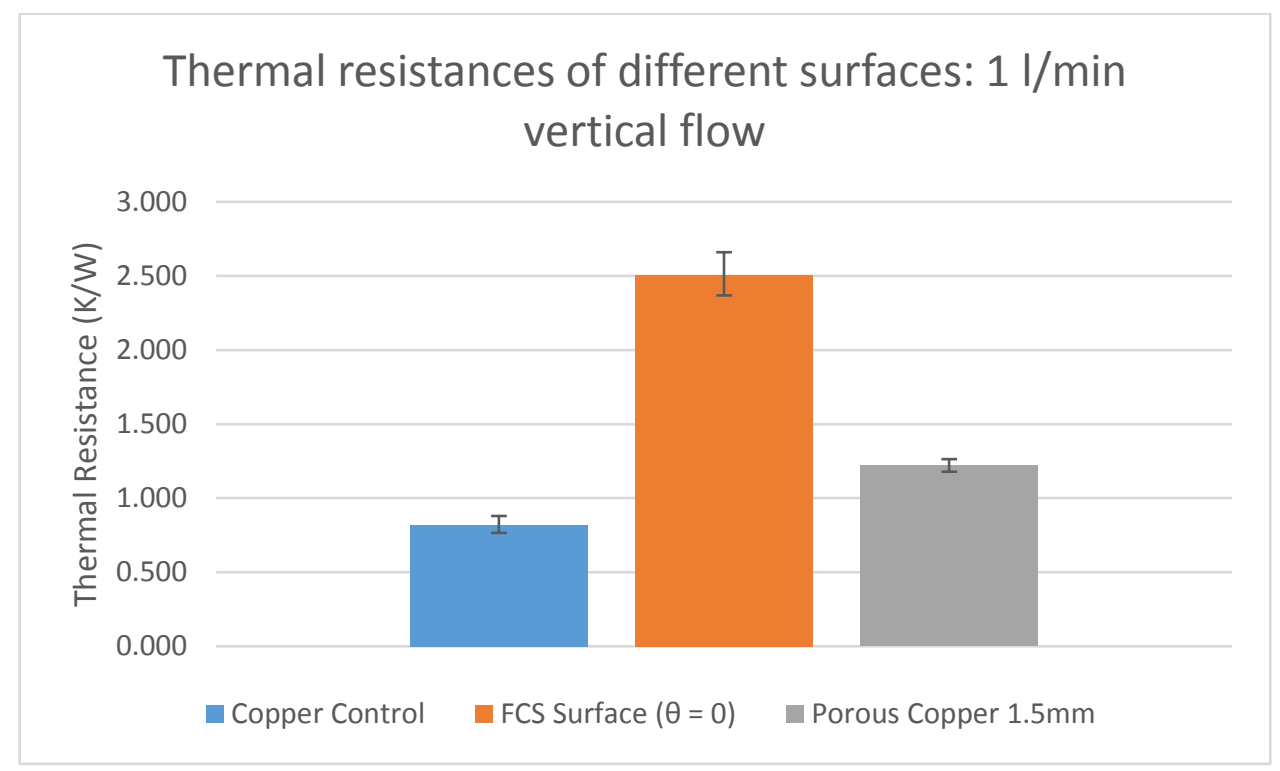

Figure 71: Comparison of thermal resistances under $1 \mathrm{l} / \mathrm{min}$ vertical flow. Only the lowest thermal resistance of each type of surface is shown.

As these two figures clearly show, the FCS surfaces and porous copper surfaces both performed significantly worse than the copper control. Even the best performance of one of 
the porous surfaces was 1.3 times worse than the equivalent copper control. This implies that there may be some additional thermal resistance not being accounted for with regards to each of these surfaces. Notably, the porous copper surfaces had lower thermal resistances than the FCS surfaces in both flow directions.

\subsubsection{Porous surfaces summary table}

\begin{tabular}{|c|c|c|c|c|c|}
\hline $\begin{array}{c}\text { Substrate } \\
\text { Details }\end{array}$ & Test setup & $\begin{array}{c}\text { Media } \\
\text { description }\end{array}$ & $\begin{array}{c}\text { Contact } \\
\text { angle }\end{array}$ & $\begin{array}{c}\text { Coolant } \\
\text { flow rate } \\
\text { (I/min) }\end{array}$ & $\begin{array}{c}\text { Thermal } \\
\text { Resistance } \\
\text { (K/W) }\end{array}$ \\
\hline $\begin{array}{c}2.5 \mathrm{~mm} \text { Porous } \\
\text { Copper }\end{array}$ & $\begin{array}{c}\text { Horizontal } \\
\text { flow }\end{array}$ & $\begin{array}{c}\text { Lost-mass } \\
\text { porous copper }\end{array}$ & N/A & 1 & 1.579 \\
\hline $\begin{array}{c}1.5 \mathrm{~mm} \text { Porous } \\
\text { Copper }\end{array}$ & $\begin{array}{c}\text { Vertical } \\
\text { flow }\end{array}$ & $\begin{array}{c}\text { Lost-mass } \\
\text { porous copper }\end{array}$ & N/A & 1 & 1.219 \\
\hline FC171205a & $\begin{array}{c}\text { Horizontal } \\
\text { flow }\end{array}$ & $\begin{array}{c}\text { Freeze-cast } \\
\text { porous silver }\end{array}$ & 0 & 1.8 & 1.956 \\
\hline FC171205a & $\begin{array}{c}\text { Vertical } \\
\text { flow }\end{array}$ & $\begin{array}{c}\text { Freeze-cast } \\
\text { porous silver }\end{array}$ & 0 & 1.8 & 2.063 \\
\hline FC171106a & $\begin{array}{c}\text { Horizontal } \\
\text { flow }\end{array}$ & $\begin{array}{c}\text { Freeze-cast } \\
\text { porous silver }\end{array}$ & 9.6 & 1.8 & 1.209 \\
\hline FC171106a & $\begin{array}{c}\text { Vertical } \\
\text { flow }\end{array}$ & $\begin{array}{c}\text { Freeze-cast } \\
\text { porous silver }\end{array}$ & 9.6 & 1.8 & 2.311 \\
\hline FC171205c & $\begin{array}{c}\text { Horizontal } \\
\text { flow }\end{array}$ & $\begin{array}{c}\text { Freeze-cast } \\
\text { porous silver }\end{array}$ & 32.8 & 1.8 & 2.893 \\
\hline FC171205c & $\begin{array}{c}\text { Vertical } \\
\text { flow }\end{array}$ & $\begin{array}{c}\text { Freeze-cast } \\
\text { porous silver }\end{array}$ & 32.8 & 1.8 & 2.264 \\
\hline
\end{tabular}

Table 8: Summary table of key results of porous surfaces.

\subsection{Evaluation of thick structures}

While the surface results are useful for thermal resistance comparisons, none of the surfaces fill the chamber sufficiently to alter the hydraulic resistance. The porous silver surfaces are very thin, between $1 \mathrm{~mm}$ and $5 \mathrm{~mm}$, and while the porous copper is thicker, between $5 \mathrm{~mm}$ and $2.5 \mathrm{~mm}$, this still barely reaches the inlet and outlet of the chamber. To remedy this, a second set of tests was performed, with each surface required to fill as much of the enclosure as possible. This was done to ensure that the liquid was flowing through the channels, not over them. 
Three new substrates were fabricated for this set of tests, and the mesh fins substrate described in 4.3.3 were included as well. This was due to issues with attempting to fabricate an FCS substrate that was thick enough. The silver contracts as it is sintered and tends to tear itself off the substrate in the process. Only one substrate (FC180601a) was successfully fabricated, and it was barely thick enough to count as a thick structure substrate. It also had edges that leaned inwards, allowing fluid to continue to flow over it rather than through it. This substrate was still included in the results, but a second substrate was fabricated over the top of the mesh fins substrate to give it support along the fins. This successfully produced a substrate that filled the chamber (FC180731a). However, it was important to know how the fins themselves might affect the thermal resistance, so before this FCS substrate was fabricated, the mesh fins substrate was tested on its own.

Because of the pressure exerted on the porous copper substrate, the power to the pump had to be reduced significantly, to the point where flow rate over the copper control substrate was $0.4 \mathrm{l} / \mathrm{min}$. Because this had not been recorded prior, the new thermal resistance for the copper control is also included.

The flow was also exclusively horizontal for these tests. This was to ensure that flow was through the porous media as much as possible.

\subsubsection{Thermal resistance}

As with the surface tests, the expectation is that the porous surfaces will have a lower thermal resistance than the copper control. The mesh fins should also have a slightly lower thermal resistance, as they have a much higher surface area, but the additional surface area has a high conductive thermal resistance due to the low thickness of the wires. 


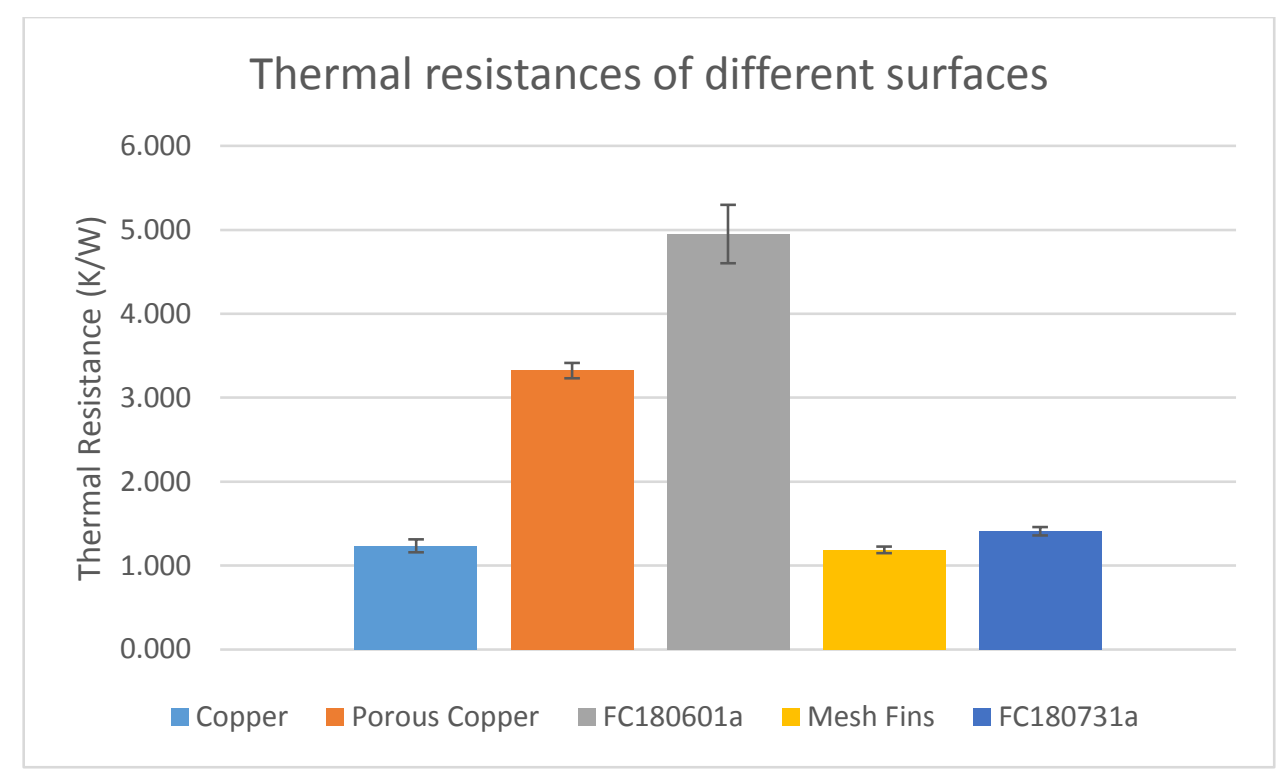

Figure 72: Comparison of thermal resistances for thick structure tests.

Figure 72 clearly shows that the porous copper and FCS structures perform significantly worse in terms of thermal resistance than the copper control, continuing the trend set in 5.3.4. This appears to confirm that some additional thermal resistance is present but not accounted for.

The mesh fins performed negligibly better, as was expected.

Most interesting is the results of the FCS supported by mesh fins. This substrate is still worse than the copper control, but not significantly. More importantly, it has 3.5 times less thermal resistance than the unsupported FCS substrate. As the unsupported FCS substrate likely has the liquid flowing over it rather than through it, this seems to imply this may be one of the issues preventing FCS surfaces from improving the thermal resistance.

\subsubsection{Hydraulic resistance}

The requirement to flow through a porous medium should significantly increase the pressure difference over the channel, and very high pressures should also lower the flow rate. As the porous copper and mesh supported FCS are the two substrates most likely to require the liquid to flow through them, these two surfaces should have the highest hydraulic resistances. 
Assuming the unsupported FCS surface is being flowed over rather than through, it should have a hydraulic resistance close to that of the copper control. The mesh fins should also have a similar hydraulic resistance.

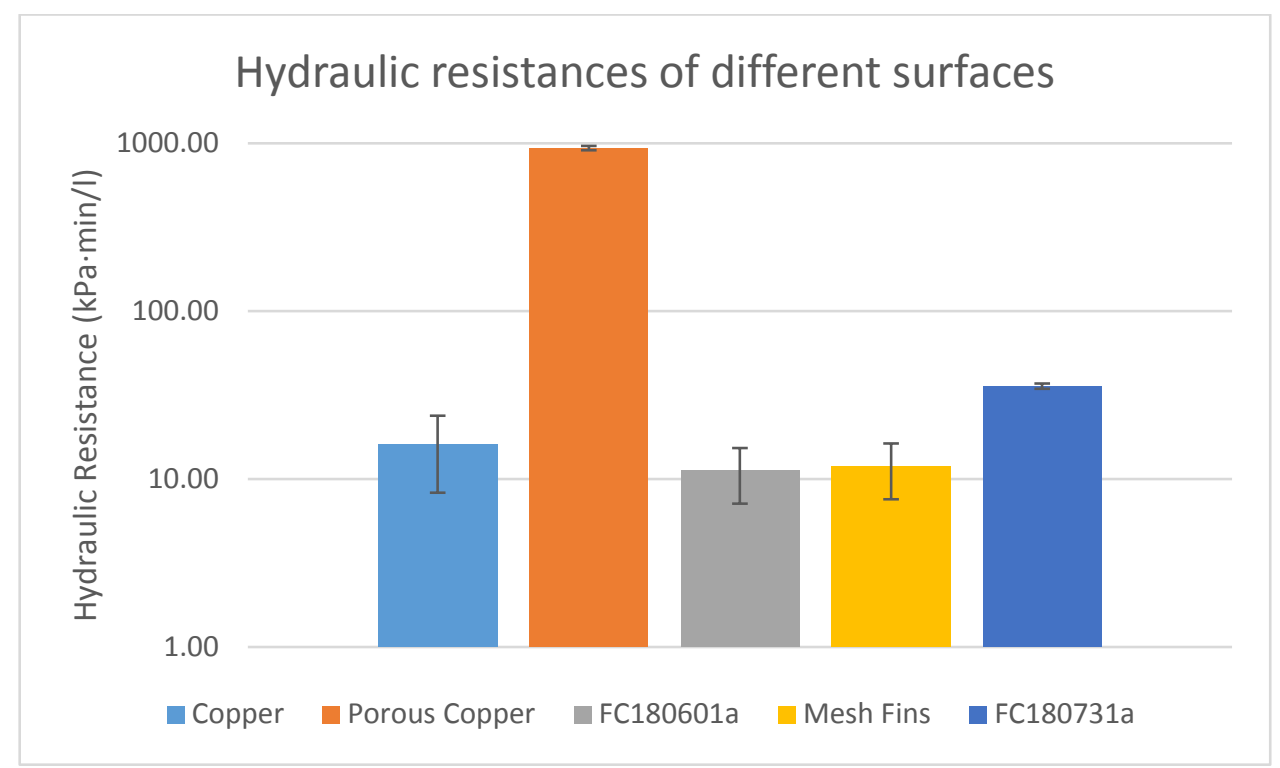

Figure 73: Comparison of hydraulic resistances for thick structure tests. Note the the scale in this case is logorythmic, due to the very high hydraulic resistance of the porous copper.

As is abundantly clear from Figure 73 , the hydraulic resistance of the porous copper dwarfs all the other hydraulic resistances. The mesh supported FCS shows a much higher hydraulic resistance than the remaining three, as expected, which confirms that the fluid is flowing through it rather than over it. This lends credence to the hypothesis that the higher thermal resistance of the FCSs in the surface tests is due to the fluid simply flowing over the silver.

The other three substrates all had similar hydraulic resistances, with the control having a slightly higher resistance than the other two. However, the differences are less than $5 \mathrm{kPa} \cdot \mathrm{min} / \mathrm{l}$, which is the error associated with the value. This is due to the large error on the pressure measurement combined with the low flow rate. No conclusions can be drawn from their hydraulic resistances with relation to each other. 
5.3.3. Thick structure summary table.

\begin{tabular}{|c|c|c|c|c|c|}
\hline $\begin{array}{l}\text { Substrate } \\
\text { Details }\end{array}$ & $\begin{array}{c}\text { Media } \\
\text { description }\end{array}$ & $\begin{array}{l}\text { Coolant } \\
\text { flow rate } \\
(\mathrm{l} / \mathrm{min})\end{array}$ & $\begin{array}{c}\text { Pressure } \\
\text { difference } \\
\quad(\mathrm{kPa})\end{array}$ & $\begin{array}{l}\text { Hydraulic } \\
\text { Resistance } \\
(\mathrm{kPa} \cdot \mathrm{min} / \mathrm{l})\end{array}$ & $\begin{array}{c}\text { Thermal } \\
\text { Resistance } \\
\text { (K/W) }\end{array}$ \\
\hline $\begin{array}{l}\text { Copper } \\
\text { Control }\end{array}$ & Flat copper & 0.4 & 10.6 & 23.81 & 1.112 \\
\hline $\begin{array}{l}\text { Thick structure } \\
\text { porous copper }\end{array}$ & $\begin{array}{l}\text { Lost-mass } \\
\text { porous copper }\end{array}$ & 0.1 & 109.6 & 922.27 & 2.354 \\
\hline FC180601a & $\begin{array}{l}\text { Freeze-cast } \\
\text { porous silver }\end{array}$ & 0.4 & 7.8 & 18.32 & 4.482 \\
\hline Mesh fins & Copper mesh & 0.4 & 8.7 & 19.82 & 1.131 \\
\hline FC180731a & $\begin{array}{c}\text { FCS on copper } \\
\text { mesh }\end{array}$ & 0.4 & 16.8 & 37.03 & 1.302 \\
\hline
\end{tabular}

Table 9: Summary table of key results of thick structures. 



\section{Conclusions}

This work describes the design, development and evaluation of a closed loop liquid cooling system with an integrated test system, as well as the use of that system to analyse the thermal and hydrodynamic characteristics of various surface structures for use in liquid cooling applications. The main results relate to the performance of the cooling and test system, followed by the results relating to the FCS surfaces and finally the results pertaining to the porous copper. In this section, important results are discussed, and areas of improvement are outlined for future work.

\subsection{Integrated system performance}

The integrated system was designed in two stages: the cooling system and the test system. The cooling system consisted of a pump, a reservoir and bubble trap, a heat exchanger, and an enclosure poised on top of a heat source, each connected to the next via flexible silicone tubing. The test system added a heat flux meter to the heat source for use in calculating the thermal resistance and introduced a flow meter and pressure sensor to measure the hydraulic resistance as well. Four more points around the system had their temperature monitored using thermocouples.

Most of the integrated system performed perfectly. The pump provided a consistent flow rate for a given input power, and the flow meter succeeded in providing this rate accurately. The heat flux meter proved to measure heat flux accurately. The thermal resistances calculated using the measured heat flux were as expected in each of the sanity checks. Additionally, the heat loss calculated from those heat flux values agreed with the expected heat loss due to the thermal leaks found using infrared imaging of the system.

However, three details were noted as places where the system could be improved in the future.

\subsubsection{Implement rigid tubing}

As was mentioned in section 3.1.6, and again in section 5.1.2, the tubing used to connect the different components of the system was flexible as it was necessary to be able to easily alter 
the system. This has the unfortunate side effect of limiting the pressure the system can handle, as above a certain pressure the tubing simply pops off the connector it is attached to. Replacing this tubing with rigid pipes, such as metal or rigid plastic, would allow for much higher pressures and flow rates to be tested.

\subsubsection{Improve insulation}

Section 5.1.5 addressed the issue of power loss between the heater power supply and the substrate. Over half the power was being lost through the wires at the bottom of the heating column, the insulation surrounding the heating column, and the surface where the substrate makes contact. This serves to lower the thermal resistance of every substrate tested using the system, which in turn means that errors have a larger effect on the results. Improving the insulation at these areas, as well as anywhere else in the system that appears to be leaking heat, would serve to increase the systems accuracy in measuring thermal resistance.

\subsubsection{Consider alternative pressure sensors}

Section 3.2.4 mentioned the large error margin on the pressure sensor being used, and this error became a problem in section 5.4.2, where what looked like a potentially significant difference in hydraulic resistance was in fact smaller than the error associated with that value. Either replacing the pressure sensor with another, more accurate model, or finding a way of increasing the accuracy of the one currently implemented, would serve to vastly improve the quality of the hydraulic resistance results.

\subsection{Silver surfaces performance}

The FCS surfaces were fabricated by a multi-step process consisting of freezing a silver/PVA/water solution on a substrate, then freeze drying this substrate, then sintering it in a furnace, and then boiling it. The surfaces have varying contact angles depending on the initial amounts of silver and PVA used but show significant variability. They also tend to deteriorate over time.

The surfaces did not perform as desired. To start with, the number of surfaces that made it to testing was far too small, which meant no solid trend could be found connecting contact 
angle to thermal resistance. Moreover, the few data points that were collected showed that the porous silver had a higher thermal resistance than the copper control, and that a lower contact angle did not necessarily result in a lower thermal resistance, as was expected.

Evidently, there are some key details where improvements can be made here.

\subsubsection{Investigate FCS surface deterioration}

As was initially covered in section 4.2.1, the surfaces' deterioration is a pretty significant problem. The current hypothesis is that the silver is reacting to form silver oxides or sulfides, changing the surface chemistry in the process, but this has yet to be confirmed. Research into why and how this deterioration occurs, and potential solutions, is highly advised.

\subsubsection{Fabricate and test more FCS surfaces}

One key problem with section 5.2.1's results is the very low sample size. While many of the tests in this project suffer from this to a degree due to the amount of time required to perform a test, the FCS surfaces suffered from this the most, with only three data points per configuration. Fabricating and testing many more FCS surfaces would allow for a much clearer relationship to be drawn between the various characteristics of the surfaces and their corresponding thermal resistances.

\subsubsection{Analyse FCS characteristics}

It is quite clear from section 5.3.4 and section 5.4.1 that the FCS substrates have a higher thermal resistance than their equivalent copper control, particularly when the liquid is not flowing through the pores of the silver. This is likely due to the presence of thermal resistances that have not been accounted for, such as an additional conductive thermal resistance between the copper and the silver. Analysing the broader thermal characteristics of these surfaces, such as their thermal conductance from surface to base, may identify where this problem is arising.

Furthermore, analysing the porous properties, such as average pore size and pore density, as well as chemical properties like the aforementioned presence of silver compounds, may identify additional characteristics which have an effect on the thermal resistance. 


\subsubsection{Experiment with freeze cast porous copper}

As was shown in section 5.3.1, the porous structure supported by mesh performed significantly better than other porous silver surfaces. However, the porous silver still performed worse than the copper control and copper mesh structures. If the hypothesis that these silver surfaces are chemically deteriorating is correct, then it may be worthwhile retrying these experiments with another metal that is less likely to deteriorate this way, such as copper. In particular, remaking the porous silver coated mesh with copper particles should give the same geometrical advantages as the porous silver, and may mitigate the disadvantages.

\subsection{Porous copper performance}

The porous copper was cut from a Versarien copper foam heat sink and soldered onto a substrate. The surface was slowly milled away, and the thermal resistance was measured both before and after the oxide layer on the surface was replaced.

These surfaces also did not perform as desired. Their thermal resistance was unpredictable both before and after oxidizing, and they always performed worse than the copper control. However, it should be noted that this is not the fault of Versarien, as these heat sinks are not designed for liquid cooling purposes. 


\section{Bibliography}

[1] I. Sauciuc, R. Prasher, J.-Y. Chang, H. Erturk, G. Chrysler, C.-P. Chiu and R. Mahajan, "Thermal Performance and Key Challenges for Future CPU Cooling Technologies," in Advances in Electronic Packaging, Parts A, B, and C, San Francisco, CA, 2005.

[2] V. J. Fesharaki, M. Dehghani, J. J. Fesharaki and H. Tavasoli, "The Effect of Temperature on Photovoltaic Cell Efficiency," in International Conference on Emerging Trends in Energy Conservation, Tehran, Iran, 2011.

[3] C. Lin, F. Wang, B. Fan, S. Ren, Y. Zhang, L. Han, S. Liu and S. Xu, "Comparative study on the heat generation behavior of lithium-ion batteries with different cathode materials using accelerating rate calorimetry," Energy Procedia, vol. 142, pp. 3369-3374, 2017.

[4] P. Rodgers, V. Eveloy and M. G. Pecht, "Limits of air-cooling: status and challenges," in Semiconductor Thermal Measurement and Management IEEE Twenty First Annual IEEE Symposium, San Jose, CA, 2005.

[5] D. B. Tuckerman and R. F. W. Pease, "High-performance heat sinking for VLSI," IEEE Electron Device Letters, vol. 2, no. 5, pp. 126-129, 1981.

[6] M. M. Waldrop, "The chips are down for Moore's law," Nature, vol. 530, no. 7589, p. 144-147, 2016.

[7] H. Lebov, "A darker shade of green: Hazards associated with lithium-ion batteries," Journal of High Technology Law, vol. 17, no. 1, pp. 78-104, 2016. 
[8] M. J. Moran, H. N. Shapiro, B. R. Munson and D. P. DeWitt, Introduction to thermal systems engineering: thermodynamics, fluid mechanics, and heat transfer, New Delhi: Wiley, 2003.

[9] W. M. Rohsenow, J. P. Hartnett and Y. I. Cho, Handbook of heat transfer, London: McGraw-Hill, 1998.

[10] J. H. Lienhard IV and J. H. Lienhard V, A heat transfer textbook, 4th ed., Cambridge, Massachusetts: Phlogiston Press, 2017.

[11] J. P. Holman, Heat transfer, Boston: McGraw-Hill, 2010.

[12] M. Kaviany, Essentials of heat transfer, Cambridge: Cambridge University Press, 2011.

[13] Y. Nakayama and R. F. Boucher, Introduction to fluid mechanics, Oxford: ButterworthHeinemann, 2000.

[14] N. Zaharia, "Passive Cooling vs. Active Cooling - What's the Difference?," Simscale, 13 March 2018. [Online]. Available: https://www.simscale.com/blog/2017/01/active-andpassive-cooling/.

[15] Delta Cooling Towers, Inc., "The Difference Between Closed and Open Circuit Cooling Tower Systems," Delta Cooling Towers, Inc., 27 October 2014. [Online]. Available: https://deltacooling.com/resources/news/the-difference-between-closed-and-opencircuit-cooling-tower-systems.

[16] M. M. Ohadi, S. V. Dessiatoun, K. Choo, M. Pecht and J. V. Lawler, “A comparison analysis of air, liquid, and two-phase cooling of data centers," 2012 28th Annual IEEE Semiconductor Thermal Measurement and Management Symposium (SEMI-THERM), pp. 58-63, 2012. 
[17] Y. A. Cengel, Introduction to Thermodynamics and Heat Transfer, McGraw-Hill Education, 2007.

[18] H. D. Young, R. A. Freedman and A. L. Ford, Sears and Zemansky's University Physics with Modern Physics, 13th Edition, Pearson, 2012.

[19] J. D. Carr, An examination of CPU cooling technologies: thermal management options for engineers, 2014.

[20] J. Basic, Turbulent history of fluid mechanics (brief essay), DOI: 10.13140/RG.2.1.3487.8485, 2016.

[21] F. M. White, Fluid Mechanics, Boston: McGraw-Hill, 2009.

[22] Product Release Europe, Hydrophilic vs Hydrophobic Coatings: What's The Difference?, Product Release Europe, 2017.

[23] Isothane, "What is the difference between Open and Closed Cell foam?," 30 January 2017. [Online]. Available: http://www.isothane.com/difference-open-closed-cellfoam/.

[24] D. A. Nield and A. Bejan, Convection in porous media, New York: Springer-Verlag, 1999.

[25] S. Prakash and S. Kumar, "Fabrication of microchannels: A review," J Engineering Manufacture, vol. 229, no. 8, pp. 1273-1288, 2015.

[26] J. Banhart, M. F. Ashby and N. A. Fleck, "Metal foams and porous metal structures," in International conference on metal foams and porous metal structures, Bremen, 1999. 
[27] J. Banhart, "Manufacture, characterisation and application of cellular metals and metal foams," Progress in Materials Science, vol. 49, no. 6, pp. 559-632, 2001.

[28] G. Gouws and N. Shortt, "Microstructured silver surfaces produced by freeze casting for enchanced phase change heat transfer," Journal of Physics Conference Series, vol. 660, no. 10.1088/1742-6596/660/1/012045, p. 012045, 2015.

[29] K. L. Scotti and D. C. Dunand, "Freeze casting - A review of processing, microstructure and Freeze casting - A review of processing, microstructure and," Progress in Materials Science, vol. 96, pp. 243-305, 2018.

[30] Z. Q. Chen, P. Cheng and T. S. Zhao, "An experimental study of two phase flow and boiling heat transfer in bi-dispersed porous channels," International Communications in Heat and Mass Transfer, vol. 27, no. 3, pp. 293-302, 2000.

[31] P.-X. Jiang, M.-H. Fan, G.-S. Si and Z.-P. Ren, "Thermal-hydraulic performance of small scale micro-channel and porous-media heat-exchangers," International Journal of Heat and Mass Transfer, vol. 44, no. 5, pp. 1039-1051, 2001.

[32] K. Boomsma, D. Poulikakos and F. Zwick, "Metal foams as compact high performance heat exchangers," Mechanics of Materials, vol. 35, no. 12, pp. 1161-1176, 2003.

[33] P.-X. Jiang, M. Li, T.-J. Lu, L. Yu and Z.-P. Ren, “Experimental research on convection heat transfer in sintered porous plate channels," International Journal of Heat and Mass Transfer, vol. 47, no. 10-11, pp. 2085-2096, 2004.

[34] H. Y. Zhang, D. Pinjala, Y. K. Joshi, T. N. Wong, K. C. Toh and M. K. Iyer, "Fluid flow and heat transfer in liquid cooled foam heat sinks for electronic packages," IEEE Transactions on Components and Packaging Technologies, vol. 28, no. 2, pp. 272-280, 2005. 
[35] G. Hetsroni, M. Gurevich and R. Rozenblit, "Sintered porous medium heat sink for cooling of high-power mini-devices," International Journal of Heat and Fluid Flow, vol. 27, no. 2, pp. 259-266, 2006.

[36] R. Singh, A. Akbarzadeh and M. Mochizuki, "Sintered porous heat sink for cooling of high-powered microprocessors for server applications," International Journal of Heat and Mass Transfer, vol. 52, no. 9-10, pp. 2289-2299, 2009.

[37] Z. M. Wan, G. Q. Guo, K. L. Su, Z. K. Tu and W. Liu, "Experimental analysis of flow and heat transfer in a miniature porous heat sink for high heat flux application," Internation Journal of Heat and Mass Transfer, vol. 55, no. 15-16, pp. 4437-4441, 2012.

[38] B. Agostini, M. Fabbri, J. E. Park, L. Wojtan, J. R. Thome and B. Michel, "State of the art of high heat flux cooling technologies," Heat Transfer Engineering, vol. 28, no. 4, pp. 258-281, 2007.

[39] G. J. Gouws, B. A. Sherson, A. Sinnathambi, R. S. Babu and C. P. Moore, "Influence of structure and wettability of porous silver surfaces on enhancing phase change heat transfer," in 17th IEEE Intersociety Conference on Thermal and Thermomechanical Phenomena in Electronic Systems (ITherm), San Diego, CA, 2018.

[40] Engineers Edge, "Thermal Properties of Metals, Conductivity, Thermal Expansion, Specific Heat," 25 February 2001. [Online]. Available: https://www.engineersedge.com/properties_of_metals.htm.

[41] Morgan Advanced Materials, "Macor Properties," Morgan Advanced Materials, 2016. [Online]. Available: http://www.morgantechnicalceramics.com/engb/products/macor-machinable-glass-ceramic/macor-properties/. 
[42] Southtile, "Ceramic Fibre Board Technical Characteristics," Southtile, [Online]. Available: https://invercargill.southtile.co.nz/files/122/file/Southtile-Ceramic-Fibreboard2-pdf.

[43] Pumps Online, "12V High Pressure Auto Diaphragm Water Pump 4L/min 100 PSI," Pumps Online, [Online]. Available: https://www.pumpsonline.co.nz/pumps/centrifugalpumps/12-volt-sprayers/12v-high-pressure-auto-diaphragm-water-pump-4I-min-100psi.html.

[44] Omega, "Flowrate Sensors FYB2000 Series," Omega, [Online]. Available: https://www.omega.com/pptst/FTB2000.html.

[45] Omega, "PX26 SERIES Pressure Transducers," Omega, [Online]. Available: https://www.omega.com/manuals/manualpdf/M1608.pdf.

[46] National Instruments, "DEVICE SPECIFICATIONS NI CDAQ-9171 NI CompactDAQ OneSlot Bus-Powered USB Chassis," National Instruments, [Online]. Available: http://www.ni.com/pdf/manuals/374037b.pdf.

[47] National Instruments, "DATASHEET NI 9212 and TB-9212," National Instruments, [Online]. Available: http://www.ni.com/pdf/manuals/374358b_02.pdf.

[48] National Instruments, "SPECIFICATIONS USB-6009," National Instruments, [Online]. Available: http://www.ni.com/pdf/manuals/375296c.pdf.

[49] Good Will Instrument Co., LTD., "PSW-Series Specifications," GW INSTEK, [Online]. Available: https://www.gwinstek.com/en-global/products/downloadSeriesSpec/1320.

[50] Topward, "6000 Series Operation Manual," Topward, [Online]. Available: https://www.topward.com.tw/data/6000.pdf. 
[51] G. Rosengarten, J. Cooper-White and G. Metcalfe, "Experimental and analytical study of the effect of contact angle on liquid convective heat transfer in microchannels," International Journal of Heat and Mass Transfer, vol. 49, no. 21-22, pp. 4161-4170, 2006.

[52] Digitech, "2.0MP USB Digital Microscope with 50x 200x Magnification," Digitech, [Online]. Available: http://www.digitech-i.com/products/20mp-usb-digital-microscope50x-200x-magnification.html.

[53] Versarien Technologies, "Low Profile Metallic Foam Heat Sinks," Versarien Technologies, 10 March 2015. [Online]. Available: http://www.versarientechnologies.co.uk/wp-content/uploads/2015/04/VTL-LowProfileHeatsinkDATASHEET-MARCH2015fv.pdf.

[54] Electrolube, "HTS Silicone Heat Transfer Compound," [Online]. Available: https://www.electrolube.com/pdf/tds/044/HTS.pdf.

[55] Arctic Silver, “Arctic Silver 5 Safety Data Sheet (SDS)," 1 August 2018. [Online]. Available: http://www.arcticsilver.com/PDF/AS5_SDS.pdf.

[56] FLIR, “FLIR T420 \& T440," September 2012. [Online]. Available: https://www.flirdirect.com/pdfs/cache/www.flir-direct.com/t440/datasheet/t440-datasheet.pdf.

[57] Corsair, "Hydro Series ${ }^{\mathrm{TM}} \mathrm{H} 110280 \mathrm{~mm}$ Extreme Performance Liquid CPU Cooler," Corsair, [Online]. Available: https://www.corsair.com/us/en/Categories/Products/LiquidCooling/Hydro-Series\%E2\%84\%A2-H110-280mm-Extreme-Performance-Liquid-CPUCooler/p/CW-9060014-WW. 

